\title{
Theoretical Study of the Microhydration the Chemical Warfare Agent Sulfur Mustard
}

\author{
Shëyhaane A. Emambocus, ${ }^{a}$ Lydia Rhyman, ${ }^{\mathrm{a}, \mathrm{b}}$ and Ponnadurai Ramasami, ${ }^{\mathrm{a}, \mathrm{b}}$ * \\ ${ }^{a}$ Computational Chemistry Group, Department of Chemistry, Faculty of Science, University of \\ Mauritius, Réduit 80837, Mauritius \\ ${ }^{b}$ Department of Chemical Sciences, University of Johannesburg, PO Box 17011, Doornfontein, \\ Johannesburg 2028, South Africa
}

*Author for correspondence email: p.ramasami@uom.ac.mu 


\section{Table of Contents}

1. Conformational search for the sulfur mustard molecule $\quad$ S3

2. Coordinates and structures of the microhydrated complexes of SM S8

2.1 Coordinates of the hydrated complexes of SM obtained from different methods S8

2.1.1 Coordinates of the complexes of SM using M06-2X/6-311++G(2d,2p) S8

2.1.2 Coordinates of the complexes of SM using B3LYP/6-311++G(2d,2p) S26

2.1.3 Coordinates of the complexes of SM using B3LYP-D3/6-311++G(2d,2p) S44

2.1.4 Coordinates of the complexes of SM using MP2/6-311++G(2d,2p) S61

2.2 Structures of complexes obtained using B3LYP/6-311++G(2d,2p) S79

2.3 Structures of complexes obtained using B3LYP-D3/6-311++G(2d,2p) S81

2.4 Structures of complexes obtained using MP2/6-311++G(2d,2p) S83

3. Effect of microhydration on the energy of SM S86

4. Effect of microhydration on the structural parameters of SM S90

5. Effect of microhydration on the infrared wavenumbers of SM and water $\quad$ S96

6. Solvation of SM using different methods S99 


\section{Conformational search for the sulfur mustard molecule}

The atom labelings and torsional angles defining the conformation of sulfur mustard (SM) are as follows:

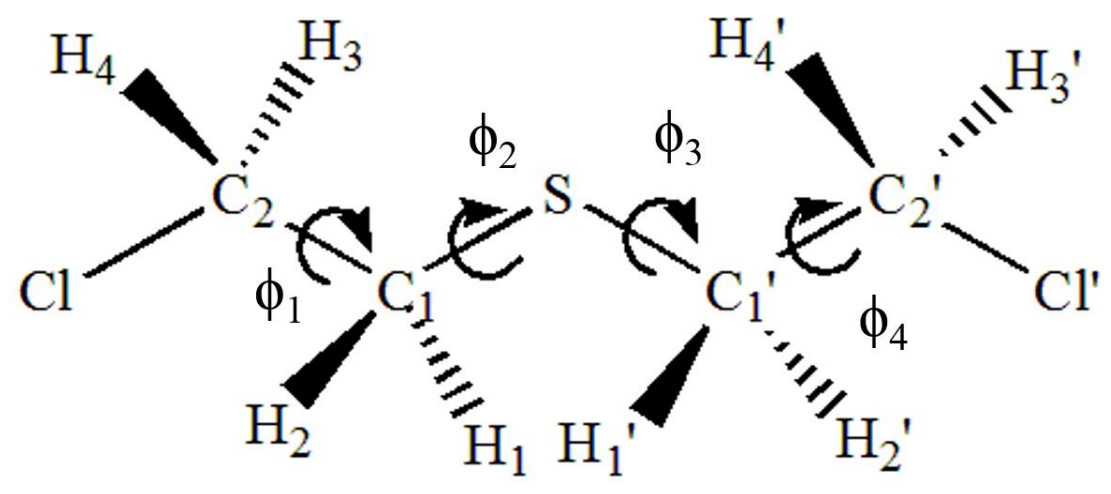

Scheme S1: Atom labelings and torsional angles of SM

The conformers of SM obtained using B3LYP/6-311++G(2d,2p) and their energy relative to one another are shown in Figure S1. The torsional angles of the optimised conformers are listed in Table S1. 


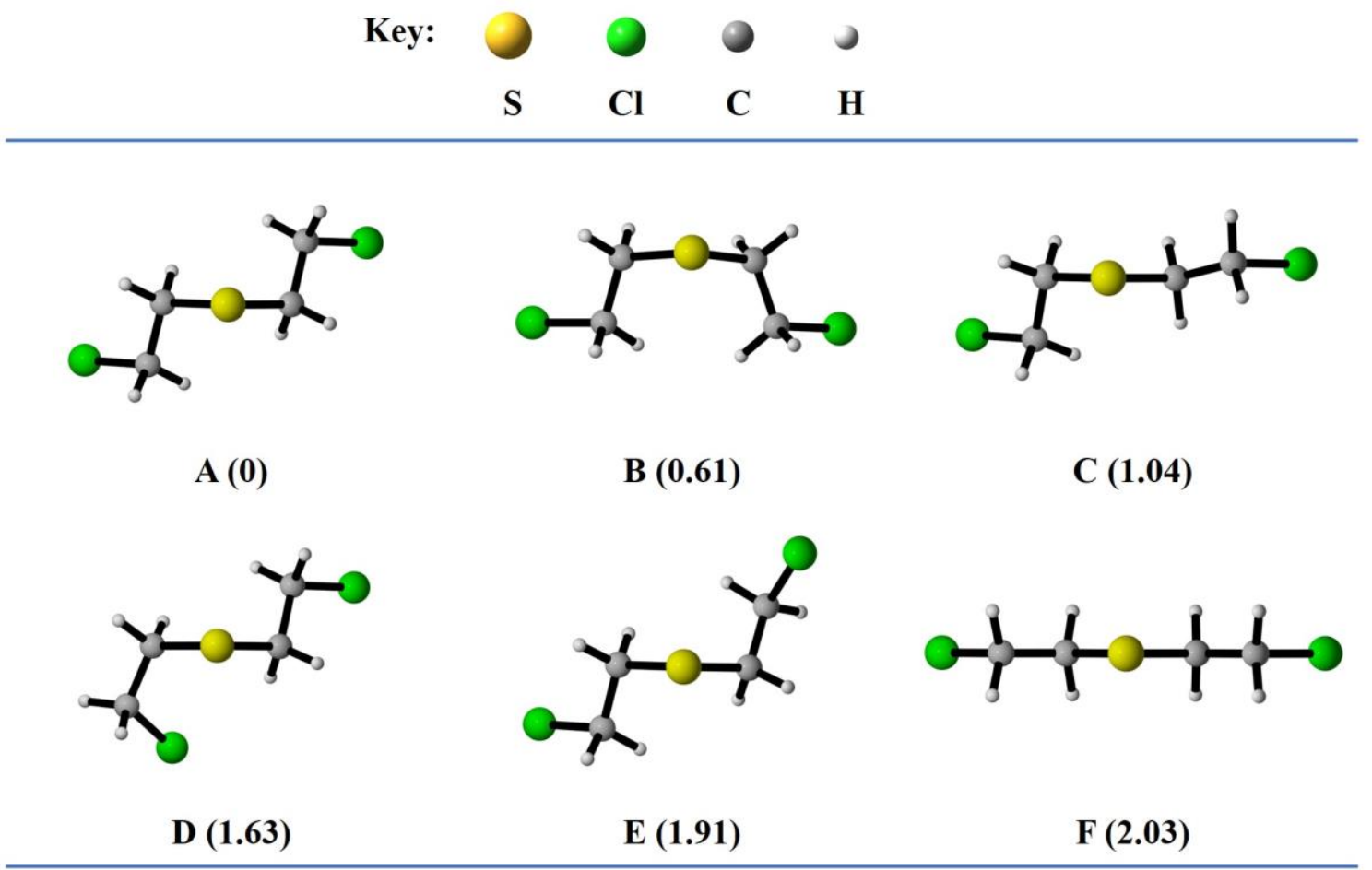

Figure S1: Conformers of SM and their relative stabilities in kcal/mol obtained at B3LYP/6-311++G(2d,2p) 


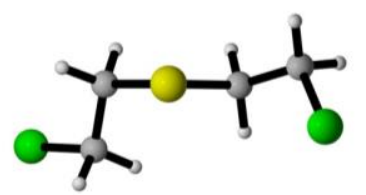

G (2.07)

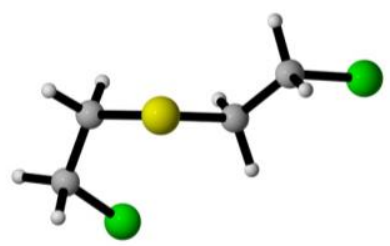

J (2.52)

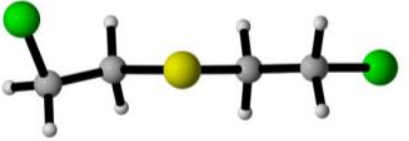

M (3.16)

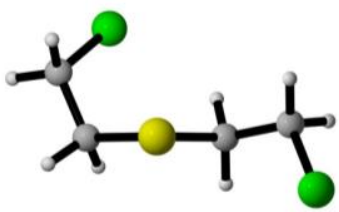

P (3.57)

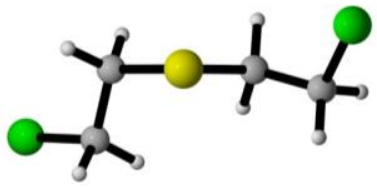

H (2.26)

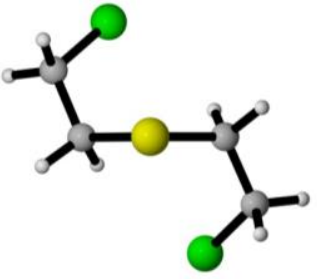

K (2.87)

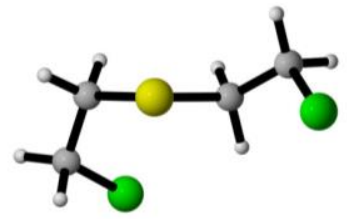

N (3.47)

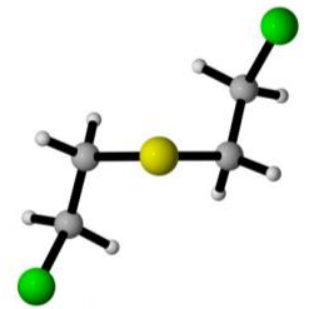

Q (4.14)

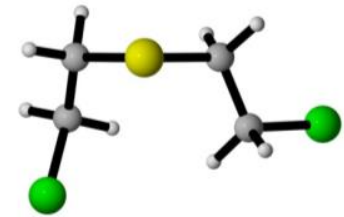

I (2.47)

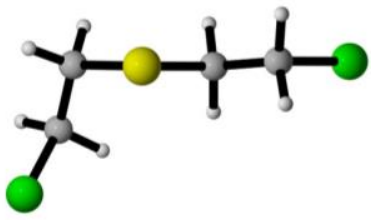

L (2.93)

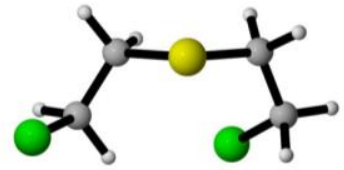

O (3.50)

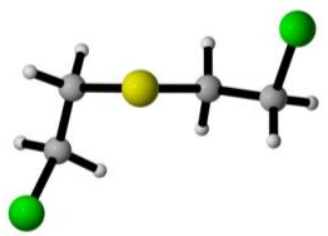

R (4.27)

Figure S1 (continued): Conformers of SM and their relative stabilities in kcal/mol obtained at B3LYP/6$311++\mathrm{G}(2 \mathrm{~d}, 2 \mathrm{p})$ 


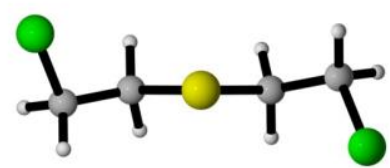

S (4.36)

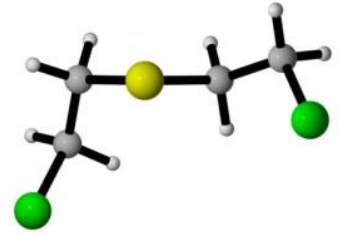

T (4.42)

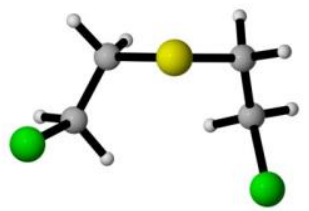

V (4.86)

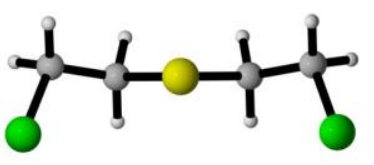

U(4.60)

Figure S1 (continued): Conformers of SM and their relative stabilities in $\mathrm{kcal} / \mathrm{mol}$ obtained at B3LYP/6$311++G(2 d, 2 p)$ 
Table S1: Torsional angles of the optimised SM conformers using B3LYP/6-311++G(2d,2p)

\begin{tabular}{ccccc}
\hline Conformers & $\boldsymbol{\phi 1} /^{\circ}$ & $\boldsymbol{\phi} \mathbf{2}^{\circ}$ & $\boldsymbol{\phi 3} /^{\circ}$ & $\mathbf{\phi 4} /^{\circ}$ \\
\hline $\mathbf{A}$ & 180 & 82 & 82 & 180 \\
$\mathbf{B}$ & -177 & -94 & 81 & 176 \\
$\mathbf{C}$ & 179 & 78 & 166 & 179 \\
$\mathbf{D}$ & -73 & 94 & 82 & 179 \\
$\mathbf{E}$ & 68 & 80 & 83 & -179 \\
$\mathbf{F}$ & 180 & 180 & 180 & 180 \\
$\mathbf{G}$ & 68 & 164 & 78 & 179 \\
$\mathbf{H}$ & -66 & -164 & 82 & -179 \\
$\mathbf{I}$ & 63 & 66 & -98 & -179 \\
$\mathbf{J}$ & -72 & 87 & 142 & 177 \\
$\mathbf{K}$ & 72 & -95 & -95 & 72 \\
$\mathbf{L}$ & 68 & 75 & 175 & 180 \\
$\mathbf{M}$ & 67 & 168 & 178 & 180 \\
$\mathbf{N}$ & 67 & 111 & -87 & 76 \\
$\mathbf{O}$ & -75 & 85 & 151 & 66 \\
$\mathbf{P}$ & 66 & 170 & -93 & 73 \\
$\mathbf{Q}$ & 68 & 78 & 78 & 68 \\
$\mathbf{R}$ & 67 & 75 & -172 & -68 \\
$\mathbf{S}$ & 68 & 169 & 169 & 68 \\
$\mathbf{T}$ & 67 & 162 & 74 & 67 \\
$\mathbf{U}$ & -68 & -171 & 171 & 68 \\
$\mathbf{V}$ & 70 & 103 & -66 & -63 \\
\hline
\end{tabular}




\section{Coordinates and structures of the microhydrated complexes of SM}

The coordinates of the hydrated complexes obtained using M06-2X, B3LYP, B3LYP-D3 and MP2 with the $6-311++\mathrm{G}(2 \mathrm{~d}, 2 \mathrm{p})$ basis set have been provided in section 3.1. Figures S2 to S4 illustrate the hydrated clusters of SM obtained using B3LYP, B3LYP-D3 and MP2, respectively with the $6-311++\mathrm{G}(2 \mathrm{~d}, 2 \mathrm{p})$ basis set.

\subsection{Coordinates of the hydrated complxes of SM obtained from different methods}

\subsubsection{Coordinates of the complexes of $S M$ using M06-2X/6-311++G(2d,2p)}

$\underline{\mathrm{G}_{0}-\text { structure of } \mathbf{A}}$

$\begin{array}{llll}\mathrm{S} & 0.000000000 & 0.000000000 & 1.708102000 \\ \mathrm{C} & 0.573763000 & 1.277367000 & 0.546129000 \\ \mathrm{H} & 1.291870000 & 1.885097000 & 1.091972000 \\ \mathrm{H} & 1.090651000 & 0.808161000 & -0.288246000 \\ \mathrm{C} & -0.585629000 & 2.123682000 & 0.070198000 \\ \mathrm{H} & -1.053331000 & 2.657817000 & 0.889820000 \\ \mathrm{H} & -1.330321000 & 1.542459000 & -0.463112000 \\ \mathrm{C} & -0.573763000 & -1.277367000 & 0.546129000 \\ \mathrm{H} & -1.291870000 & -1.885097000 & 1.091972000 \\ \mathrm{H} & -1.090651000 & -0.808161000 & -0.288246000 \\ \mathrm{C} & 0.585629000 & -2.123682000 & 0.070198000 \\ \mathrm{H} & 1.330321000 & -1.542459000 & -0.463112000 \\ \mathrm{H} & 1.053331000 & -2.657817000 & 0.889820000 \\ \mathrm{Cl} & 0.000000000 & -3.379692000 & -1.093718000 \\ \mathrm{Cl} & 0.000000000 & 3.379692000 & -1.093718000\end{array}$

$\underline{\mathrm{G}_{1}}$ - Structure of A1-1
S $\quad 0.000000000$
$0.000000000 \quad 2.087660000$
C $\quad 0.598070000$
1.258913000
0.918057000 


$\begin{array}{lrrr}\mathrm{H} & 1.244318000 & 1.920050000 & 1.491952000 \\ \mathrm{H} & 1.190617000 & 0.786478000 & 0.139555000 \\ \mathrm{C} & -0.552290000 & 2.029021000 & 0.309570000 \\ \mathrm{H} & -1.062544000 & 2.643968000 & 1.043223000 \\ \mathrm{H} & -1.257170000 & 1.374003000 & -0.189340000 \\ \mathrm{C} & -0.598070000 & -1.258913000 & 0.918057000 \\ \mathrm{H} & -1.244318000 & -1.920050000 & 1.491952000 \\ \mathrm{H} & -1.190617000 & -0.786478000 & 0.139555000 \\ \mathrm{C} & 0.552290000 & -2.029021000 & 0.309570000 \\ \mathrm{H} & 1.257170000 & -1.374003000 & -0.189340000 \\ \mathrm{H} & 1.062544000 & -2.643968000 & 1.043223000 \\ \mathrm{Cl} & -0.062298000 & -3.150849000 & -0.968890000 \\ \mathrm{Cl} & 0.062298000 & 3.150849000 & -0.968890000 \\ \mathrm{O} & 0.000000000 & 0.000000000 & -1.902166000 \\ \mathrm{H} & -0.104910000 & -0.765796000 & -2.472629000 \\ \mathrm{H} & 0.104910000 & 0.765796000 & -2.472629000\end{array}$

$\underline{G}_{1}-$ Structure of A1-2

$\begin{array}{cccc}\mathrm{S} & -0.257927000 & -1.550499000 & 0.259206000 \\ \mathrm{C} & -1.695556000 & -0.510516000 & 0.659887000 \\ \mathrm{H} & -2.257546000 & -1.038247000 & 1.427562000 \\ \mathrm{H} & -1.361790000 & 0.434706000 & 1.084214000 \\ \mathrm{C} & -2.557557000 & -0.282533000 & -0.562477000 \\ \mathrm{H} & -2.963698000 & -1.215383000 & -0.938907000 \\ \mathrm{H} & -2.014246000 & 0.219654000 & -1.356922000 \\ \mathrm{C} & 0.865411000 & -0.293081000 & -0.422937000 \\ \mathrm{H} & 1.575363000 & -0.825178000 & -1.051550000\end{array}$




$\begin{array}{llll}\mathrm{H} & 0.305470000 & 0.406611000 & -1.040590000 \\ \mathrm{C} & 1.598473000 & 0.417829000 & 0.692178000 \\ \mathrm{H} & 0.927417000 & 0.931924000 & 1.373022000 \\ \mathrm{H} & 2.237581000 & -0.267697000 & 1.237411000 \\ \mathrm{Cl} & 2.683133000 & 1.698160000 & 0.008905000 \\ \mathrm{Cl} & -3.957416000 & 0.774396000 & -0.151660000 \\ \mathrm{O} & 3.904887000 & -1.232636000 & -0.439981000 \\ \mathrm{H} & 4.722289000 & -1.703149000 & -0.609755000 \\ \mathrm{H} & 4.115080000 & -0.297817000 & -0.525005000\end{array}$

$\underline{\mathrm{G}}_{1}-$ Structure of A1-3

$\begin{array}{lrrr}\mathrm{S} & -0.047486000 & 0.935198000 & 1.365759000 \\ \mathrm{C} & -1.296092000 & 0.548754000 & 0.096870000 \\ \mathrm{H} & -1.859660000 & 1.464410000 & -0.069696000 \\ \mathrm{H} & -0.798852000 & 0.303139000 & -0.839080000 \\ \mathrm{C} & -2.208187000 & -0.565697000 & 0.561114000 \\ \mathrm{H} & -2.763217000 & -0.279887000 & 1.448403000 \\ \mathrm{H} & -1.664498000 & -1.484684000 & 0.757770000 \\ \mathrm{C} & 1.184175000 & -0.357714000 & 1.006626000 \\ \mathrm{H} & 1.784149000 & -0.463555000 & 1.908240000 \\ \mathrm{H} & 0.673025000 & -1.303186000 & 0.835644000 \\ \mathrm{C} & 2.059589000 & 0.008122000 & -0.172958000 \\ \mathrm{H} & 1.492161000 & 0.162506000 & -1.084803000 \\ \mathrm{H} & 2.645744000 & 0.898795000 & 0.029269000 \\ \mathrm{Cl} & 3.224698000 & -1.325591000 & -0.496578000 \\ \mathrm{Cl} & -3.416233000 & -0.949139000 & -0.715129000 \\ \mathrm{O} & 0.592498000 & 2.570129000 & -1.388480000\end{array}$




$\begin{array}{llll}\mathrm{H} & 0.742868000 & 3.478046000 & -1.656166000 \\ \mathrm{H} & 0.587254000 & 2.569843000 & -0.424779000\end{array}$

$\underline{\mathrm{G}}_{2}$ - Structure of A2-1

$\begin{array}{lrrr}\mathrm{O} & -0.007723000 & 1.661612000 & 0.183998000 \\ \mathrm{H} & -0.772651000 & 2.245464000 & 0.071259000 \\ \mathrm{H} & 0.773077000 & 2.214723000 & 0.097016000 \\ \mathrm{O} & -2.534919000 & 2.852358000 & -0.210268000 \\ \mathrm{H} & -2.825661000 & 3.162098000 & -1.069776000 \\ \mathrm{H} & -2.986168000 & 2.012562000 & -0.062831000 \\ \mathrm{~S} & 0.739456000 & -2.254116000 & -0.155295000 \\ \mathrm{C} & -0.731067000 & -1.456335000 & 0.561060000 \\ \mathrm{H} & -1.209748000 & -2.223901000 & 1.165994000 \\ \mathrm{H} & -0.438112000 & -0.631155000 & 1.205879000 \\ \mathrm{C} & 1.706246000 & -0.799345000 & -0.658801000 \\ \mathrm{H} & 1.061968000 & -0.083481000 & -1.160937000 \\ \mathrm{H} & 2.447032000 & -1.163727000 & -1.367677000 \\ \mathrm{C} & 2.375769000 & -0.157773000 & 0.534405000 \\ \mathrm{H} & 3.131511000 & -0.795619000 & 0.979754000 \\ \mathrm{H} & 1.655283000 & 0.155876000 & 1.281652000 \\ \mathrm{C} & -1.665855000 & -0.965762000 & -0.520240000 \\ \mathrm{H} & -1.237722000 & -0.136627000 & -1.073986000 \\ \mathrm{Cl} & 3.237870000 & 1.349842000 & 0.020004000 \\ \mathrm{H} & -1.971071000 & -1.762758000 & -1.189246000 \\ \mathrm{Cl} & -3.192485000 & -0.335964000 & 0.234069000\end{array}$




\section{$\underline{\mathrm{G}}_{2}-$ Structure of A2-2}

$\begin{array}{lrrr}\mathrm{S} & -0.775084000 & -2.257375000 & 0.036896000 \\ \mathrm{C} & -1.727317000 & -0.819434000 & 0.613894000 \\ \mathrm{H} & -2.483702000 & -1.211979000 & 1.290376000 \\ \mathrm{H} & -1.079163000 & -0.145758000 & 1.167562000 \\ \mathrm{C} & 0.726015000 & -1.441372000 & -0.589552000 \\ \mathrm{H} & 0.461184000 & -0.572691000 & -1.187240000 \\ \mathrm{H} & 1.213583000 & -2.175618000 & -1.227753000 \\ \mathrm{C} & 1.633558000 & -1.038964000 & 0.549635000 \\ \mathrm{H} & 1.916281000 & -1.886166000 & 1.164790000 \\ \mathrm{H} & 1.196494000 & -0.250297000 & 1.152360000 \\ \mathrm{C} & -2.370477000 & -0.100048000 & -0.550130000 \\ \mathrm{H} & -1.633234000 & 0.248867000 & -1.264635000 \\ \mathrm{Cl} & 3.187131000 & -0.366048000 & -0.107980000 \\ \mathrm{H} & -3.116085000 & -0.713881000 & -1.043932000 \\ \mathrm{Cl} & -3.227926000 & 1.381213000 & 0.036647000 \\ \mathrm{O} & 0.054067000 & 1.649398000 & -0.052696000 \\ \mathrm{H} & 0.812432000 & 2.250333000 & 0.004683000 \\ \mathrm{H} & -0.729240000 & 2.175985000 & 0.128723000 \\ \mathrm{O} & 2.576605000 & 2.876000000 & 0.148809000 \\ \mathrm{H} & 2.941797000 & 3.338223000 & -0.607852000 \\ \mathrm{H} & 2.978457000 & 1.998895000 & 0.133260000\end{array}$

$\underline{\mathrm{G}_{2}-\text { Structure of A2-3 }}$
S $\quad 0.066721000$
$0.133676000 \quad-1.715916000$
$\begin{array}{llll}\text { C } & -1.174416000 & -0.951519000 & -0.942273000\end{array}$
$\mathrm{H} \quad-1.770755000 \quad-1.351580000 \quad-1.759885000$ 


$\begin{array}{lrrr}\mathrm{H} & -0.669530000 & -1.786164000 & -0.459839000 \\ \mathrm{C} & 1.321901000 & 0.224300000 & -0.397371000 \\ \mathrm{H} & 0.832068000 & 0.330189000 & 0.569064000 \\ \mathrm{H} & 1.883935000 & 1.138464000 & -0.583125000 \\ \mathrm{C} & 2.238411000 & -0.978628000 & -0.442827000 \\ \mathrm{H} & 2.767704000 & -1.042707000 & -1.387759000 \\ \mathrm{H} & 1.704093000 & -1.906895000 & -0.264354000 \\ \mathrm{C} & -2.048884000 & -0.203352000 & 0.040632000 \\ \mathrm{H} & -1.488193000 & 0.206985000 & 0.875154000 \\ \mathrm{Cl} & 3.484460000 & -0.850312000 & 0.848967000 \\ \mathrm{H} & -2.603312000 & 0.590327000 & -0.451548000 \\ \mathrm{Cl} & -3.269063000 & -1.331896000 & 0.734962000 \\ \mathrm{O} & -0.364931000 & 3.141294000 & -0.472080000 \\ \mathrm{H} & -0.443128000 & 2.357064000 & -1.035269000 \\ \mathrm{H} & -1.029549000 & 3.760349000 & -0.779486000 \\ \mathrm{O} & -0.356219000 & 1.797266000 & 2.004940000 \\ \mathrm{H} & -0.341880000 & 2.442678000 & 1.282896000 \\ \mathrm{H} & 0.176393000 & 2.166715000 & 2.710148000\end{array}$

$\underline{\mathrm{G}}_{2}$ - Structure of A2-4

$\begin{array}{lrrr}\mathrm{O} & 0.424246000 & 1.839671000 & -1.970724000 \\ \mathrm{H} & 0.989437000 & 2.345580000 & -1.368751000 \\ \mathrm{H} & 0.658135000 & 2.117918000 & -2.856991000 \\ \mathrm{O} & 1.826079000 & 2.815296000 & 0.270355000 \\ \mathrm{H} & 1.828130000 & 3.696078000 & 0.649359000 \\ \mathrm{H} & 1.242036000 & 2.279983000 & 0.829428000 \\ \mathrm{~S} & -0.105473000 & 0.466022000 & 1.572824000\end{array}$




$\begin{array}{llll}\mathrm{C} & 0.968388000 & -0.901305000 & 1.027512000 \\ \mathrm{H} & 1.546076000 & -1.196012000 & 1.901234000 \\ \mathrm{H} & 0.347989000 & -1.747253000 & 0.738416000 \\ \mathrm{C} & -1.377016000 & 0.444902000 & 0.268002000 \\ \mathrm{H} & -0.905673000 & 0.311319000 & -0.702808000 \\ \mathrm{H} & -1.829826000 & 1.434401000 & 0.271000000 \\ \mathrm{C} & -2.415385000 & -0.616857000 & 0.558770000 \\ \mathrm{H} & -2.921326000 & -0.431437000 & 1.500513000 \\ \mathrm{H} & -1.987868000 & -1.614934000 & 0.571026000 \\ \mathrm{C} & 1.881989000 & -0.484214000 & -0.104766000 \\ \mathrm{H} & 1.332025000 & -0.147874000 & -0.978234000 \\ \mathrm{Cl} & -3.676441000 & -0.617736000 & -0.723251000 \\ \mathrm{H} & 2.572203000 & 0.295235000 & 0.204074000 \\ \mathrm{Cl} & 2.880310000 & -1.893691000 & -0.618961000\end{array}$

$\underline{\mathrm{G}_{2}-\text { Structure of A2-5 }}$
S $\quad-0.115123000$
$1.118500000 \quad 0.518975000$
$\begin{array}{llll}\text { C } & -1.627093000 & 0.612548000 & -0.360874000\end{array}$
$\begin{array}{llll}\mathrm{H} & -1.969577000 & 1.479915000 & -0.921431000\end{array}$
$\mathrm{H} \quad-1.394973000 \quad-0.176165000 \quad-1.073846000$
$\begin{array}{llll}\text { C } & 0.681359000 & -0.502616000 & 0.759671000\end{array}$
$\begin{array}{llll}\mathrm{H} & -0.071194000 & -1.228273000 & 1.062308000\end{array}$
$\begin{array}{llll}\mathrm{H} & 1.389670000 & -0.360097000 & 1.570254000\end{array}$
$\begin{array}{llll}\text { C } & 1.407678000 & -0.944214000 & -0.490992000\end{array}$
H $\quad 2.221834000 \quad-0.267821000 \quad-0.726639000$
H $\quad 0.743941000 \quad-1.039899000 \quad-1.345184000$
$\begin{array}{llll}\text { C } & -2.693243000 & 0.166064000 & 0.616057000\end{array}$ 


$\begin{array}{llll}\mathrm{H} & -2.376480000 & -0.689633000 & 1.204438000 \\ \mathrm{Cl} & 2.136316000 & -2.571242000 & -0.230669000 \\ \mathrm{H} & -2.981725000 & 0.973481000 & 1.280718000 \\ \mathrm{Cl} & -4.174733000 & -0.340276000 & -0.271616000 \\ \mathrm{O} & 3.115992000 & 1.345253000 & 1.099422000 \\ \mathrm{H} & 4.030175000 & 1.221368000 & 1.357391000 \\ \mathrm{H} & 3.133714000 & 1.788642000 & 0.240121000 \\ \mathrm{O} & 2.313447000 & 2.242646000 & -1.436758000 \\ \mathrm{H} & 2.283991000 & 3.129548000 & -1.799872000 \\ \mathrm{H} & 1.437960000 & 2.074848000 & -1.057486000\end{array}$

\section{$\underline{\mathrm{G}}_{2}$ - Structure of A2-6}

$\begin{array}{lrrr}\mathrm{S} & 0.512356000 & 1.377057000 & 0.310914000 \\ \mathrm{C} & -0.514861000 & 0.013492000 & -0.318949000 \\ \mathrm{H} & -1.232427000 & 0.456961000 & -1.003067000 \\ \mathrm{H} & 0.106649000 & -0.692974000 & -0.866108000 \\ \mathrm{C} & 2.050706000 & 0.462227000 & 0.639105000 \\ \mathrm{H} & 1.820289000 & -0.506915000 & 1.079228000 \\ \mathrm{H} & 2.604415000 & 1.037730000 & 1.378082000 \\ \mathrm{C} & 2.869572000 & 0.303736000 & -0.622949000 \\ \mathrm{H} & 3.181224000 & 1.266547000 & -1.013794000 \\ \mathrm{H} & 2.332078000 & -0.240029000 & -1.393637000 \\ \mathrm{C} & -1.225618000 & -0.653906000 & 0.834741000 \\ \mathrm{H} & -0.536500000 & -1.083337000 & 1.555028000 \\ \mathrm{Cl} & 4.371121000 & -0.635429000 & -0.286686000 \\ \mathrm{H} & -1.902341000 & 0.039727000 & 1.321223000 \\ \mathrm{Cl} & -2.234059000 & -2.044832000 & 0.238532000\end{array}$




$\begin{array}{lrrr}\mathrm{O} & -2.589929000 & 2.120413000 & 0.151153000 \\ \mathrm{H} & -2.952240000 & 2.921970000 & 0.529636000 \\ \mathrm{H} & -3.333519000 & 1.638286000 & -0.238959000 \\ \mathrm{O} & -4.327920000 & 0.189696000 & -0.926473000 \\ \mathrm{H} & -3.880410000 & -0.610965000 & -0.625801000 \\ \mathrm{H} & -4.470962000 & 0.070359000 & -1.866958000\end{array}$

$\underline{\mathrm{G}_{2}-}$ - Structure of A2-7

$\begin{array}{llll}\text { O } & -2.659073000 & 2.095305000 & 0.404285000\end{array}$

$\begin{array}{llll}\mathrm{H} & -3.202192000 & 1.677496000 & -0.278764000\end{array}$

$\mathrm{H} \quad-2.555635000 \quad 3.011946000 \quad 0.146613000$

$\begin{array}{llll}\text { O } & -3.858127000 & 0.281790000 & -1.408044000\end{array}$

H $\quad-3.589275000 \quad-0.549665000 \quad-0.997125000$

$\mathrm{H} \quad-4.791462000 \quad 0.186362000 \quad-1.606336000$

$\begin{array}{llll}\text { S } & 0.475908000 & 1.344793000 & 0.370167000\end{array}$

C $\quad-0.551219000 \quad-0.024015000 \quad-0.249753000$

$\mathrm{H} \quad-1.258676000 \quad 0.405196000 \quad-0.953484000$

H $\quad 0.074752000 \quad-0.741082000 \quad-0.777803000$

$\begin{array}{llll}\text { C } & 2.034709000 & 0.449341000 & 0.650593000\end{array}$

$\begin{array}{llll}\mathrm{H} & 1.826995000 & -0.521129000 & 1.098885000\end{array}$

$\begin{array}{llll}\mathrm{H} & 2.603077000 & 1.032835000 & 1.371964000\end{array}$

$\begin{array}{llll}\text { C } & 2.817698000 & 0.297797000 & -0.634899000\end{array}$

$\mathrm{H} \quad 3.106880000 \quad 1.263470000 \quad-1.035959000$

$\mathrm{H} \quad 2.263839000 \quad-0.253258000 \quad-1.388792000$

$\begin{array}{llll}\text { C } & -1.276235000 & -0.671178000 & 0.906886000\end{array}$

$\mathrm{H} \quad-0.594923000 \quad-1.113625000 \quad 1.626804000$

$\mathrm{Cl} \quad 4.337982000 \quad-0.624047000 \quad-0.340291000$ 


$\begin{array}{llll}\mathrm{H} & -1.938962000 & 0.035701000 & 1.393531000 \\ \mathrm{Cl} & -2.312750000 & -2.039444000 & 0.309167000\end{array}$

$\underline{\mathrm{G}}_{3}-$ Structure of A3-1

$\begin{array}{lrrr}\mathrm{S} & -0.017404000 & -2.521503000 & 0.032029000 \\ \mathrm{C} & -1.352731000 & -1.346455000 & 0.492063000 \\ \mathrm{H} & -2.027053000 & -1.923813000 & 1.121598000 \\ \mathrm{H} & -0.948068000 & -0.537058000 & 1.094577000 \\ \mathrm{C} & 1.289558000 & -1.393714000 & -0.594678000 \\ \mathrm{H} & 0.842804000 & -0.631525000 & -1.228086000 \\ \mathrm{H} & 1.925720000 & -2.013036000 & -1.223735000 \\ \mathrm{C} & 2.083350000 & -0.775727000 & 0.537142000 \\ \mathrm{H} & 2.641845000 & -1.516935000 & 1.097582000 \\ \mathrm{H} & 1.484211000 & -0.166090000 & 1.205139000 \\ \mathrm{C} & -2.073769000 & -0.842658000 & -0.740813000 \\ \mathrm{H} & -1.421981000 & -0.298693000 & -1.415480000 \\ \mathrm{Cl} & 3.357000000 & 0.371760000 & -0.148564000 \\ \mathrm{H} & -2.571776000 & -1.640854000 & -1.279529000 \\ \mathrm{Cl} & -3.395307000 & 0.348397000 & -0.285996000 \\ \mathrm{O} & -0.995167000 & 2.817441000 & 0.334931000 \\ \mathrm{H} & -0.362003000 & 2.683364000 & -0.392059000 \\ \mathrm{H} & -1.802775000 & 2.360397000 & 0.071079000 \\ \mathrm{O} & 0.974285000 & 2.189246000 & -1.622757000 \\ \mathrm{H} & 1.283920000 & 2.840935000 & -2.257801000 \\ \mathrm{H} & 1.770504000 & 1.821547000 & -1.209443000 \\ \mathrm{O} & 0.095575000 & 1.214639000 & 2.316215000 \\ \mathrm{H} & -0.299091000 & 1.881484000 & 1.717794000 \\ \mathrm{H} & 0.137435000 & 1.622371000 & 3.184028000\end{array}$




\section{$\underline{\mathrm{G}}_{3}-$ Structure of A3-2}

$\begin{array}{lrrr}\mathrm{S} & -0.477128000 & -2.472069000 & -0.098983000 \\ \mathrm{C} & -1.620560000 & -1.191889000 & 0.555885000 \\ \mathrm{H} & -2.307001000 & -1.733637000 & 1.203546000 \\ \mathrm{H} & -1.071914000 & -0.479089000 & 1.165762000 \\ \mathrm{C} & -2.370462000 & -0.498282000 & -0.561992000 \\ \mathrm{H} & -2.965135000 & -1.192734000 & -1.144666000 \\ \mathrm{H} & -1.712880000 & 0.071547000 & -1.208185000 \\ \mathrm{C} & 0.987681000 & -1.489845000 & -0.614471000 \\ \mathrm{H} & 1.513533000 & -2.125255000 & -1.324094000 \\ \mathrm{H} & 0.672264000 & -0.589795000 & -1.136478000 \\ \mathrm{C} & 1.873288000 & -1.169354000 & 0.571403000 \\ \mathrm{H} & 1.417734000 & -0.461248000 & 1.255020000 \\ \mathrm{H} & 2.178355000 & -2.062503000 & 1.104241000 \\ \mathrm{Cl} & 3.440134000 & -0.382588000 & 0.013162000 \\ \mathrm{Cl} & -3.552469000 & 0.720903000 & 0.129002000 \\ \mathrm{O} & -0.196430000 & 2.071326000 & -1.359467000 \\ \mathrm{H} & -0.612142000 & 2.730344000 & -1.921262000 \\ \mathrm{H} & 0.714047000 & 2.388978000 & -1.180545000 \\ \mathrm{O} & -0.075061000 & 1.951459000 & 1.445256000 \\ \mathrm{H} & -0.457062000 & 2.037013000 & 0.552859000 \\ \mathrm{H} & -0.720990000 & 2.324816000 & 2.050554000 \\ \mathrm{O} & 2.174724000 & 2.765645000 & -0.145777000 \\ \mathrm{H} & 2.779862000 & 2.012830000 & -0.151957000 \\ \mathrm{H} & 1.669519000 & 2.669264000 & 0.677093000\end{array}$


G-ㅡㄴ Structure of A3-3

\begin{tabular}{|c|c|c|c|}
\hline & -0.510092000 & 00 & 5000 \\
\hline & 000 & 0 & 000 \\
\hline & 2000 & 0 & 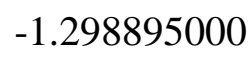 \\
\hline & 0.60486 & -0 . & 000 \\
\hline & 000 & -1 . & 0 \\
\hline & 5000 & -2. & 000 \\
\hline & 000 & -0 . & 000 \\
\hline & -1.6 & -1. & 000 \\
\hline $\mathrm{H}$ & -2.261 & -1 . & 000 \\
\hline $\mathrm{H}$ & -1.036618000 & 9000 & 000 \\
\hline $\mathrm{C}$ & $-2.441<$ & 3000 & 000 \\
\hline $\mathrm{H}$ & -1.824697000 & 739000 & 4000 \\
\hline $\mathrm{H}$ & -3.073907000 & 391000 & 35000 \\
\hline & -3.572632000 & 000 & 2000 \\
\hline $\mathrm{Cl}$ & 2000 & -0. & 5000 \\
\hline 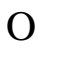 & 2.225449000 & & 7000 \\
\hline $\mathrm{H}$ & 2.804 & 000 & 7000 \\
\hline $\mathrm{H}$ & 2000 & 000 & 4000 \\
\hline $\mathrm{O}$ & 0.205 & 000 & 4000 \\
\hline $\mathrm{H}$ & 1.03 & 0 & 000 \\
\hline $\mathrm{H}$ & -0.063949000 & 000 & 56000 \\
\hline $\mathrm{O}$ & -0.3489 & 2.13334 & 1000 \\
\hline $\mathrm{H}$ & -1.077596000 & 2.633138000 & -1.585872000 \\
\hline & 0.514447000 & 2.100635000 & -0.249203000 \\
\hline
\end{tabular}




\section{$\underline{\mathrm{G}}_{3}-$ Structure of A3-4}

$\begin{array}{lrrr}\mathrm{S} & 1.687868000 & -2.201919000 & -0.124186000 \\ \mathrm{C} & 2.253790000 & -0.535445000 & -0.647807000 \\ \mathrm{H} & 3.042080000 & -0.724368000 & -1.373811000 \\ \mathrm{H} & 1.445268000 & -0.011043000 & -1.147112000 \\ \mathrm{C} & 2.780648000 & 0.265790000 & 0.523081000 \\ \mathrm{H} & 3.642373000 & -0.201113000 & 0.986755000 \\ \mathrm{H} & 2.013742000 & 0.463332000 & 1.262464000 \\ \mathrm{C} & 0.032543000 & -1.875055000 & 0.600346000 \\ \mathrm{H} & -0.196951000 & -2.778773000 & 1.161605000 \\ \mathrm{H} & 0.077289000 & -1.041076000 & 1.295002000 \\ \mathrm{C} & -1.006786000 & -1.630184000 & -0.472367000 \\ \mathrm{H} & -0.889952000 & -0.659308000 & -0.939521000 \\ \mathrm{H} & -1.030968000 & -2.423033000 & -1.210775000 \\ \mathrm{Cl} & -2.691295000 & -1.616423000 & 0.277449000 \\ \mathrm{Cl} & 3.343449000 & 1.916468000 & -0.042624000 \\ \mathrm{O} & -0.136386000 & 1.638788000 & -0.008627000 \\ \mathrm{H} & -0.922016000 & 2.209353000 & 0.095123000 \\ \mathrm{H} & 0.592969000 & 2.239468000 & -0.191035000 \\ \mathrm{O} & -2.477386000 & 3.179834000 & 0.207607000 \\ \mathrm{H} & -3.193732000 & 2.551424000 & -0.005331000 \\ \mathrm{H} & -2.701010000 & 3.552492000 & 1.064042000 \\ \mathrm{O} & -4.350101000 & 1.193467000 & -0.386075000 \\ \mathrm{H} & -4.740605000 & 1.150481000 & -1.263127000 \\ \mathrm{H} & -3.881194000 & 0.354762000 & -0.262076000 \\ & & \end{array}$




\section{$\underline{\mathrm{G}}_{3}-$ Structure of A3-5}

$\begin{array}{lrrr}\mathrm{S} & 0.002066000 & -2.534484000 & -0.042651000 \\ \mathrm{C} & 1.227156000 & -1.369320000 & -0.764045000 \\ \mathrm{H} & 1.740599000 & -1.923547000 & -1.546737000 \\ \mathrm{H} & 0.702676000 & -0.541421000 & -1.235012000 \\ \mathrm{C} & -1.263119000 & -1.371367000 & 0.608693000 \\ \mathrm{H} & -0.778721000 & -0.535755000 & 1.108624000 \\ \mathrm{H} & -1.818321000 & -1.932108000 & 1.357706000 \\ \mathrm{C} & -2.175347000 & -0.922689000 & -0.513473000 \\ \mathrm{H} & -2.776012000 & -1.733801000 & -0.908898000 \\ \mathrm{H} & -1.638733000 & -0.437759000 & -1.321260000 \\ \mathrm{C} & 2.203797000 & -0.876501000 & 0.283360000 \\ \mathrm{H} & 1.721264000 & -0.352921000 & 1.101535000 \\ \mathrm{Cl} & -3.380604000 & 0.336215000 & 0.084070000 \\ \mathrm{H} & 2.828758000 & -1.676350000 & 0.664682000 \\ \mathrm{Cl} & 3.362451000 & 0.338469000 & -0.469212000 \\ \mathrm{O} & 1.026334000 & 2.870405000 & 0.215430000 \\ \mathrm{H} & 0.276768000 & 2.782412000 & -0.398949000 \\ \mathrm{H} & 1.770109000 & 2.416862000 & -0.198626000 \\ \mathrm{O} & -1.293608000 & 2.457222000 & -1.381593000 \\ \mathrm{H} & -1.687632000 & 3.179613000 & -1.878204000 \\ \mathrm{H} & -2.013791000 & 2.065565000 & -0.865550000 \\ \mathrm{O} & 0.391957000 & 1.127434000 & 2.274160000 \\ \mathrm{H} & 0.386400000 & 1.520764000 & 3.149571000 \\ \mathrm{H} & 0.609790000 & 1.849328000 & 1.649765000\end{array}$




\section{$\underline{\mathrm{G}}_{3}-$ Structure of A3-6}

$\begin{array}{lrrr}\mathrm{S} & 0.442329000 & -1.069383000 & 0.449171000 \\ \mathrm{C} & 2.019406000 & -0.721202000 & -0.425070000 \\ \mathrm{H} & 2.253533000 & -1.631342000 & -0.973131000 \\ \mathrm{H} & 1.875832000 & 0.073807000 & -1.152339000 \\ \mathrm{C} & 3.130658000 & -0.385706000 & 0.549424000 \\ \mathrm{H} & 3.333425000 & -1.207990000 & 1.226652000 \\ \mathrm{H} & 2.923362000 & 0.512449000 & 1.121208000 \\ \mathrm{C} & -0.214659000 & 0.611364000 & 0.797619000 \\ \mathrm{H} & -1.003795000 & 0.445110000 & 1.528002000 \\ \mathrm{H} & 0.562190000 & 1.210787000 & 1.266070000 \\ \mathrm{C} & -0.772514000 & 1.293166000 & -0.436316000 \\ \mathrm{H} & -0.030932000 & 1.415224000 & -1.218554000 \\ \mathrm{H} & -1.646510000 & 0.785648000 & -0.829437000 \\ \mathrm{Cl} & -1.315294000 & 2.984660000 & -0.013004000 \\ \mathrm{Cl} & 4.685325000 & -0.059963000 & -0.347100000 \\ \mathrm{O} & -3.487680000 & -0.867981000 & 1.408152000 \\ \mathrm{H} & -4.191187000 & -0.817147000 & 2.059632000 \\ \mathrm{H} & -3.834261000 & -0.466997000 & 0.591427000 \\ \mathrm{O} & -2.456792000 & -2.676927000 & -0.574313000 \\ \mathrm{H} & -2.772950000 & -2.411600000 & 0.304706000 \\ \mathrm{H} & -1.516829000 & -2.451578000 & -0.563447000 \\ \mathrm{O} & -3.744620000 & -0.360970000 & -1.333389000 \\ \mathrm{H} & -4.441447000 & -0.350988000 & -1.993613000 \\ \mathrm{H} & -3.342825000 & -1.253798000 & -1.349681000\end{array}$




\section{$\underline{\mathrm{G}}_{3}-$ Structure of A3-7}

$\begin{array}{lrrr}\mathrm{O} & 0.126540000 & -1.614092000 & 2.139242000 \\ \mathrm{H} & 0.706901000 & -2.159364000 & 1.586465000 \\ \mathrm{H} & 0.081255000 & -2.051206000 & 2.992822000 \\ \mathrm{O} & 1.832043000 & -2.661022000 & 0.076627000 \\ \mathrm{H} & 2.366171000 & -3.444595000 & -0.080832000 \\ \mathrm{H} & 2.424923000 & -1.898097000 & -0.029607000 \\ \mathrm{~S} & -0.532229000 & 2.464820000 & 0.245217000 \\ \mathrm{C} & 0.972500000 & 1.478176000 & 0.614542000 \\ \mathrm{H} & 1.566550000 & 2.120677000 & 1.261781000 \\ \mathrm{H} & 0.710467000 & 0.585140000 & 1.176194000 \\ \mathrm{C} & 1.729544000 & 1.146282000 & -0.653139000 \\ \mathrm{H} & 2.051716000 & 2.032009000 & -1.188190000 \\ \mathrm{H} & 1.182737000 & 0.479102000 & -1.310177000 \\ \mathrm{C} & -1.655823000 & 1.219591000 & -0.503432000 \\ \mathrm{H} & -2.424015000 & 1.806283000 & -1.003564000 \\ \mathrm{H} & -1.121914000 & 0.649189000 & -1.257349000 \\ \mathrm{C} & -2.266506000 & 0.306182000 & 0.538764000 \\ \mathrm{H} & -1.529704000 & -0.270413000 & 1.086757000 \\ \mathrm{H} & -2.911002000 & 0.842067000 & 1.226884000 \\ \mathrm{Cl} & -3.339081000 & -0.941269000 & -0.280846000 \\ \mathrm{Cl} & 3.292758000 & 0.242522000 & -0.249228000 \\ \mathrm{O} & -0.179832000 & -1.622362000 & -1.792501000 \\ \mathrm{H} & -1.087355000 & -1.816972000 & -1.533469000 \\ \mathrm{H} & 0.378132000 & -2.153827000 & -1.207293000\end{array}$


$\underline{G}_{3}-$ Structure of A3-8

$\begin{array}{lrrr}\mathrm{S} & -1.307452000 & -0.734670000 & 1.366202000 \\ \mathrm{C} & -0.204636000 & 0.127369000 & 0.178881000 \\ \mathrm{H} & 0.721375000 & -0.443309000 & 0.165770000 \\ \mathrm{H} & -0.636773000 & 0.094605000 & -0.818456000 \\ \mathrm{C} & 0.055427000 & 1.551284000 & 0.625332000 \\ \mathrm{H} & 0.573952000 & 1.587796000 & 1.576579000 \\ \mathrm{H} & -0.846996000 & 2.150529000 & 0.676768000 \\ \mathrm{C} & -2.968918000 & -0.183106000 & 0.810500000 \\ \mathrm{H} & -3.620927000 & -0.301755000 & 1.673177000 \\ \mathrm{H} & -2.944508000 & 0.874772000 & 0.559353000 \\ \mathrm{C} & -3.467776000 & -1.015651000 & -0.352995000 \\ \mathrm{H} & -2.830371000 & -0.931512000 & -1.226599000 \\ \mathrm{H} & -3.575142000 & -2.060289000 & -0.082921000 \\ \mathrm{Cl} & -5.120150000 & -0.448177000 & -0.882959000 \\ \mathrm{Cl} & 1.139431000 & 2.423197000 & -0.574155000 \\ \mathrm{O} & 5.068056000 & -1.831364000 & -1.484843000 \\ \mathrm{H} & 5.366262000 & -1.576722000 & -2.361373000 \\ \mathrm{H} & 4.268678000 & -1.303877000 & -1.302474000 \\ \mathrm{O} & 5.808972000 & -0.590396000 & 0.919408000 \\ \mathrm{H} & 5.851796000 & -1.136750000 & 0.113033000 \\ \mathrm{H} & 6.032356000 & -1.172639000 & 1.649737000 \\ \mathrm{O} & 3.221059000 & -0.207189000 & -0.112716000 \\ \mathrm{H} & 2.962854000 & 0.696961000 & -0.326672000 \\ \mathrm{H} & 3.999617000 & -0.146215000 & 0.470690000\end{array}$




\section{$\underline{\mathrm{G}}_{3}-$ Structure of A3-9}

$\begin{array}{lrrr}\mathrm{S} & 0.586561000 & -1.156901000 & 1.546399000 \\ \mathrm{C} & -0.967821000 & -0.598777000 & 0.738858000 \\ \mathrm{H} & -1.586604000 & -0.224349000 & 1.552056000 \\ \mathrm{H} & -0.744268000 & 0.235498000 & 0.078350000 \\ \mathrm{C} & -1.661746000 & -1.736563000 & 0.022595000 \\ \mathrm{H} & -1.942593000 & -2.539367000 & 0.694945000 \\ \mathrm{H} & -1.080880000 & -2.133956000 & -0.802381000 \\ \mathrm{C} & 1.765805000 & -1.238165000 & 0.135060000 \\ \mathrm{H} & 2.542500000 & -1.927808000 & 0.459316000 \\ \mathrm{H} & 1.266243000 & -1.687169000 & -0.719856000 \\ \mathrm{C} & 2.356596000 & 0.111869000 & -0.220696000 \\ \mathrm{H} & 1.614379000 & 0.828916000 & -0.555865000 \\ \mathrm{H} & 2.924171000 & 0.529692000 & 0.604266000 \\ \mathrm{Cl} & 3.542338000 & -0.088849000 & -1.594896000 \\ \mathrm{Cl} & -3.232842000 & -1.154347000 & -0.729072000 \\ \mathrm{O} & -2.852238000 & 2.280314000 & -1.239508000 \\ \mathrm{H} & -3.024964000 & 2.520893000 & -2.153939000 \\ \mathrm{H} & -3.042787000 & 1.335267000 & -1.178351000 \\ \mathrm{O} & -0.073283000 & 2.441104000 & -0.373768000 \\ \mathrm{H} & -0.034469000 & 2.595405000 & 0.580884000 \\ \mathrm{H} & -1.000113000 & 2.557369000 & -0.632192000 \\ \mathrm{O} & 0.299009000 & 2.101023000 & 2.515144000 \\ \mathrm{H} & 0.631534000 & 1.193462000 & 2.440178000 \\ \mathrm{H} & 0.886529000 & 2.551183000 & 3.127815000\end{array}$




\subsubsection{Coordinates of the complexes of $S M$ using B3LYP/6-311++G(2d,2p)}

$\begin{array}{lrrr}\mathrm{G}_{0}-\text { structure of A } & & \\ \mathrm{S} & 0.000000000 & 0.000000000 & 1.687869000 \\ \mathrm{C} & 0.569226000 & 1.313227000 & 0.536308000 \\ \mathrm{H} & 1.276294000 & 1.910612000 & 1.106935000 \\ \mathrm{H} & 1.103131000 & 0.863526000 & -0.296504000 \\ \mathrm{C} & -0.592677000 & 2.160954000 & 0.063631000 \\ \mathrm{H} & -1.079071000 & 2.678665000 & 0.881971000 \\ \mathrm{H} & -1.322477000 & 1.595448000 & -0.503910000 \\ \mathrm{C} & -0.569226000 & -1.313227000 & 0.536308000 \\ \mathrm{H} & -1.276294000 & -1.910612000 & 1.106935000 \\ \mathrm{H} & -1.103131000 & -0.863526000 & -0.296504000 \\ \mathrm{C} & 0.592677000 & -2.160954000 & 0.063631000 \\ \mathrm{H} & 1.322477000 & -1.595448000 & -0.503910000 \\ \mathrm{H} & 1.079071000 & -2.678665000 & 0.881971000 \\ \mathrm{Cl} & 0.000000000 & -3.474783000 & -1.075946000 \\ \mathrm{Cl} & 0.000000000 & 3.474783000 & -1.075946000\end{array}$

\section{$\underline{\mathrm{G}_{1}-\text { Structure of A1-1 }}$}

$\begin{array}{llll}\text { S } & -0.070661000 & 2.034634000 & 0.025185000 \\ \text { C } & -1.336218000 & 0.849080000 & -0.583451000 \\ \text { H } & -1.980851000 & 1.435074000 & -1.235345000 \\ \text { H } & -0.862481000 & 0.074423000 & -1.179623000 \\ \text { C } & -2.129719000 & 0.242745000 & 0.554852000 \\ \text { H } & -2.656258000 & 0.995040000 & 1.131512000 \\ \text { H } & -1.511059000 & -0.364842000 & 1.204657000\end{array}$




$\begin{array}{llll}\mathrm{C} & 1.258960000 & 0.907929000 & 0.603109000 \\ \mathrm{H} & 1.860380000 & 1.495951000 & 1.293090000 \\ \mathrm{H} & 0.822747000 & 0.078693000 & 1.151481000 \\ \mathrm{C} & 2.095984000 & 0.411384000 & -0.556841000 \\ \mathrm{H} & 1.504979000 & -0.124176000 & -1.289870000 \\ \mathrm{H} & 2.652964000 & 1.208916000 & -1.035408000 \\ \mathrm{Cl} & 3.349867000 & -0.797070000 & 0.027475000 \\ \mathrm{Cl} & -3.411248000 & -0.887294000 & -0.098138000 \\ \mathrm{O} & 0.255332000 & -2.212450000 & 0.069493000 \\ \mathrm{H} & 1.186703000 & -2.447378000 & 0.147730000 \\ \mathrm{H} & -0.219775000 & -3.038879000 & -0.051866000\end{array}$

$\underline{\mathrm{G}}_{1}-$ Structure of A1-2
$\begin{array}{llll}\text { S } & -0.296646000 & -1.518838000 & 0.358445000\end{array}$
$\begin{array}{llll}\text { C } & -1.766207000 & -0.463583000 & 0.672120000\end{array}$
$\begin{array}{llll}\mathrm{H} & -2.328652000 & -0.967724000 & 1.455030000\end{array}$
$\mathrm{H} \quad-1.453573000 \quad 0.502218000 \quad 1.062253000$
$\begin{array}{llll}\text { C } & -2.612331000 & -0.306821000 & -0.575158000\end{array}$
$\mathrm{H} \quad-2.997470000 \quad-1.259408000 \quad-0.921687000$
$\begin{array}{llll}\mathrm{H} & -2.075864000 & 0.181606000 & -1.381583000\end{array}$
$\begin{array}{llll}\text { C } & 0.891265000 & -0.314092000 & -0.354592000\end{array}$
H $\quad 1.612512000 \quad-0.910582000 \quad-0.907991000$
$\begin{array}{llll}\mathrm{H} & 0.379604000 & 0.342649000 & -1.054265000\end{array}$
$\begin{array}{llll}\text { C } & 1.584999000 & 0.467467000 & 0.740752000\end{array}$
$\begin{array}{llll}\mathrm{H} & 0.899205000 & 1.052285000 & 1.343712000\end{array}$
$\begin{array}{llll}\mathrm{H} & 2.182668000 & -0.176679000 & 1.374743000\end{array}$
$\begin{array}{llll}\mathrm{Cl} & 2.752452000 & 1.691877000 & 0.022824000\end{array}$ 


$\begin{array}{lrrr}\mathrm{Cl} & -4.067239000 & 0.742689000 & -0.233819000 \\ \mathrm{O} & 4.133980000 & -1.277645000 & -0.580432000 \\ \mathrm{H} & 5.008384000 & -1.610777000 & -0.797193000 \\ \mathrm{H} & 4.212709000 & -0.316451000 & -0.576506000\end{array}$

\section{$\underline{\mathrm{G}_{1} \text { - Structure of A1-3 }}$}

$\begin{array}{lrrr}\mathrm{S} & -0.041555000 & 1.146177000 & 0.923429000 \\ \mathrm{C} & -1.399381000 & 0.616650000 & -0.196064000 \\ \mathrm{H} & -1.840338000 & 1.535452000 & -0.576393000 \\ \mathrm{H} & -0.987762000 & 0.073159000 & -1.042611000 \\ \mathrm{C} & -2.434690000 & -0.206521000 & 0.543826000 \\ \mathrm{H} & -2.903466000 & 0.359804000 & 1.340993000 \\ \mathrm{H} & -2.022415000 & -1.125367000 & 0.947024000 \\ \mathrm{C} & 1.054855000 & -0.331613000 & 0.936530000 \\ \mathrm{H} & 1.682267000 & -0.205288000 & 1.816358000 \\ \mathrm{H} & 0.452213000 & -1.224181000 & 1.087662000 \\ \mathrm{C} & 1.904750000 & -0.439527000 & -0.314504000 \\ \mathrm{H} & 1.309097000 & -0.556905000 & -1.213580000 \\ \mathrm{H} & 2.570770000 & 0.407511000 & -0.432258000 \\ \mathrm{Cl} & 2.955283000 & -1.927005000 & -0.217186000 \\ \mathrm{Cl} & -3.771591000 & -0.709268000 & -0.588235000 \\ \mathrm{O} & 2.211664000 & 2.868077000 & -0.954779000 \\ \mathrm{H} & 2.384749000 & 3.797919000 & -0.786804000 \\ \mathrm{H} & 1.450484000 & 2.637159000 & -0.403592000\end{array}$




\section{$\underline{\mathrm{G}}_{2}$ - Structure of A2-1}

$\begin{array}{lrrr}\mathrm{S} & 0.681407000 & -2.217061000 & 0.064922000 \\ \mathrm{C} & 1.735794000 & -0.852441000 & -0.570505000 \\ \mathrm{H} & 2.433810000 & -1.326757000 & -1.257205000 \\ \mathrm{H} & 1.126477000 & -0.147056000 & -1.126805000 \\ \mathrm{C} & -0.836424000 & -1.333176000 & 0.604510000 \\ \mathrm{H} & -0.568646000 & -0.405558000 & 1.101575000 \\ \mathrm{H} & -1.309493000 & -1.995080000 & 1.326771000 \\ \mathrm{C} & -1.753271000 & -1.077889000 & -0.571505000 \\ \mathrm{H} & -2.021362000 & -1.990061000 & -1.091683000 \\ \mathrm{H} & -1.345218000 & -0.351694000 & -1.264612000 \\ \mathrm{C} & 2.470566000 & -0.156732000 & 0.555096000 \\ \mathrm{H} & 1.791339000 & 0.328712000 & 1.245322000 \\ \mathrm{Cl} & -3.356990000 & -0.366096000 & -0.002433000 \\ \mathrm{H} & 3.137163000 & -0.827019000 & 1.086168000 \\ \mathrm{Cl} & 3.527503000 & 1.183419000 & -0.111813000 \\ \mathrm{O} & 0.029969000 & 1.910051000 & 0.033797000 \\ \mathrm{H} & -0.795522000 & 2.424514000 & 0.020786000 \\ \mathrm{H} & 0.734893000 & 2.516235000 & -0.209559000 \\ \mathrm{O} & -2.617372000 & 2.905340000 & -0.006965000 \\ \mathrm{H} & -2.981699000 & 3.332981000 & 0.772938000 \\ \mathrm{H} & -3.003745000 & 2.017567000 & -0.020484000\end{array}$

$\underline{\mathrm{G}_{2}-\text { Structure of A2-2 }}$

$\begin{array}{lrrr}\mathrm{O} & -0.118113000 & 2.167837000 & 1.326799000 \\ \mathrm{H} & -0.859336000 & 2.532505000 & 0.811598000 \\ \mathrm{H} & -0.056764000 & 2.709010000 & 2.117495000\end{array}$




$\begin{array}{lrrr}\mathrm{O} & -2.378161000 & 2.844477000 & -0.226080000 \\ \mathrm{H} & -2.298606000 & 3.237409000 & -1.099495000 \\ \mathrm{H} & -2.761642000 & 1.966140000 & -0.363232000 \\ \mathrm{~S} & 0.641412000 & -2.131058000 & 0.541794000 \\ \mathrm{C} & -0.900530000 & -1.142570000 & 0.678393000 \\ \mathrm{H} & -1.432958000 & -1.567842000 & 1.526807000 \\ \mathrm{H} & -0.663833000 & -0.107980000 & 0.914629000 \\ \mathrm{C} & 1.734924000 & -1.047522000 & -0.462513000 \\ \mathrm{H} & 1.180273000 & -0.632101000 & -1.300737000 \\ \mathrm{H} & 2.488894000 & -1.718412000 & -0.869668000 \\ \mathrm{C} & 2.379454000 & 0.044902000 & 0.366322000 \\ \mathrm{H} & 2.970018000 & -0.365379000 & 1.178085000 \\ \mathrm{H} & 1.660848000 & 0.763005000 & 0.745903000 \\ \mathrm{C} & -1.722981000 & -1.274823000 & -0.584873000 \\ \mathrm{H} & -1.232055000 & -0.851388000 & -1.453933000 \\ \mathrm{Cl} & 3.541509000 & 1.003472000 & -0.672386000 \\ \mathrm{H} & -2.003941000 & -2.301992000 & -0.786850000 \\ \mathrm{Cl} & -3.319656000 & -0.364912000 & -0.438146000\end{array}$

\section{$\underline{\mathrm{G}}_{2}$ - Structure of A2-3}

$\begin{array}{lrrr}\mathrm{S} & 0.093389000 & 0.223040000 & -1.641956000 \\ \mathrm{C} & -1.135198000 & -0.959393000 & -0.946025000 \\ \mathrm{H} & -1.680103000 & -1.341775000 & -1.806570000 \\ \mathrm{H} & -0.604864000 & -1.794841000 & -0.495773000 \\ \mathrm{C} & 1.398461000 & 0.260003000 & -0.344906000 \\ \mathrm{H} & 0.937774000 & 0.356444000 & 0.635118000 \\ \mathrm{H} & 1.956169000 & 1.174793000 & -0.536107000\end{array}$




$\begin{array}{lrrr}\mathrm{C} & 2.310143000 & -0.945956000 & -0.445248000 \\ \mathrm{H} & 2.808921000 & -0.997277000 & -1.406956000 \\ \mathrm{H} & 1.789579000 & -1.878953000 & -0.256950000 \\ \mathrm{C} & -2.079027000 & -0.308236000 & 0.044524000 \\ \mathrm{H} & -1.573855000 & 0.093654000 & 0.915505000 \\ \mathrm{Cl} & 3.627097000 & -0.849082000 & 0.811502000 \\ \mathrm{H} & -2.673752000 & 0.470569000 & -0.421150000 \\ \mathrm{Cl} & -3.269064000 & -1.547131000 & 0.662494000 \\ \mathrm{O} & -0.595076000 & 3.293314000 & -0.461152000 \\ \mathrm{H} & -0.549348000 & 2.477913000 & -0.988757000 \\ \mathrm{H} & -1.319572000 & 3.808002000 & -0.826648000 \\ \mathrm{O} & -0.517338000 & 1.905589000 & 2.018257000 \\ \mathrm{H} & -0.565863000 & 2.565414000 & 1.306288000 \\ \mathrm{H} & -0.172830000 & 2.363313000 & 2.788458000\end{array}$

$\underline{\mathrm{G}_{2}-\text { Structure of A2-4 }}$
$\mathrm{S}$
$-0.717256000-1.464382000$
0.242568000
$\begin{array}{llll}\text { C } & 0.410525000 & -0.163587000 & -0.395810000\end{array}$
H $\quad 1.129409000 \quad-0.688802000 \quad-1.018301000$
$\begin{array}{llll}\mathrm{H} & -0.146345000 & 0.538058000 & -1.012247000\end{array}$
$\begin{array}{llll}\text { C } & -2.226556000 & -0.492747000 & 0.629628000\end{array}$
$\begin{array}{llll}\mathrm{H} & -1.951838000 & 0.452602000 & 1.092107000\end{array}$
$\begin{array}{llll}\mathrm{H} & -2.770116000 & -1.075581000 & 1.370139000\end{array}$
$\begin{array}{llll}\text { C } & -3.076571000 & -0.275557000 & -0.605719000\end{array}$
$\mathrm{H} \quad-3.426192000 \quad-1.213357000 \quad-1.022945000$
$\begin{array}{llll}\mathrm{H} & -2.557483000 & 0.290191000 & -1.371945000\end{array}$
$\begin{array}{llll}\text { C } & 1.116208000 & 0.527332000 & 0.749880000\end{array}$ 


$\begin{array}{llll}\mathrm{H} & 0.435559000 & 1.002306000 & 1.447498000 \\ \mathrm{Cl} & -4.570311000 & 0.692974000 & -0.195400000 \\ \mathrm{H} & 1.784429000 & -0.150585000 & 1.266408000 \\ \mathrm{Cl} & 2.173041000 & 1.904470000 & 0.124617000 \\ \mathrm{O} & 3.159888000 & -1.976099000 & -0.138007000 \\ \mathrm{H} & 3.506511000 & -2.804268000 & 0.201436000 \\ \mathrm{H} & 3.925244000 & -1.384560000 & -0.238656000 \\ \mathrm{O} & 5.000036000 & 0.168287000 & -0.383493000 \\ \mathrm{H} & 4.304150000 & 0.826572000 & -0.242300000 \\ \mathrm{H} & 5.375313000 & 0.370841000 & -1.244849000\end{array}$

$\underline{\mathrm{G}}_{2}-$ Structure of A2-5

$\begin{array}{lrrr}\mathrm{O} & 3.174906000 & -1.970947000 & -0.055360000 \\ \mathrm{H} & 3.924282000 & -1.387388000 & -0.264195000 \\ \mathrm{H} & 3.460075000 & -2.860613000 & -0.275107000 \\ \mathrm{O} & 4.959208000 & 0.174539000 & -0.564463000 \\ \mathrm{H} & 4.252037000 & 0.822972000 & -0.433834000 \\ \mathrm{H} & 5.637040000 & 0.401635000 & 0.078024000 \\ \mathrm{~S} & -0.717760000 & -1.465397000 & 0.248593000 \\ \mathrm{C} & 0.411220000 & -0.165669000 & -0.389570000 \\ \mathrm{H} & 1.130027000 & -0.689687000 & -1.013255000 \\ \mathrm{H} & -0.145190000 & 0.537600000 & -1.004503000 \\ \mathrm{C} & -2.227724000 & -0.492986000 & 0.630610000 \\ \mathrm{H} & -1.953549000 & 0.451979000 & 1.094248000 \\ \mathrm{H} & -2.773765000 & -1.075690000 & 1.369403000 \\ \mathrm{C} & -3.074247000 & -0.274472000 & -0.606877000 \\ \mathrm{H} & -3.423272000 & -1.211763000 & -1.025739000\end{array}$




$\begin{array}{lrrr}\mathrm{H} & -2.552956000 & 0.291730000 & -1.371262000 \\ \mathrm{C} & 1.120054000 & 0.523167000 & 0.755487000 \\ \mathrm{H} & 0.440997000 & 0.996362000 & 1.455891000 \\ \mathrm{Cl} & -4.568585000 & 0.694397000 & -0.199513000 \\ \mathrm{H} & 1.790003000 & -0.154755000 & 1.270103000 \\ \mathrm{Cl} & 2.171509000 & 1.902955000 & 0.126772000\end{array}$

$\underline{\mathrm{G}_{2}-\text { Structure of A2-6 }}$

$\begin{array}{lrrr}\mathrm{O} & -2.837866000 & 2.391420000 & -0.569205000 \\ \mathrm{H} & -1.947078000 & 2.781417000 & -0.567648000 \\ \mathrm{H} & -3.206069000 & 2.572095000 & -1.437180000 \\ \mathrm{O} & -0.114135000 & 3.129898000 & -0.205715000 \\ \mathrm{H} & 0.016353000 & 3.855999000 & 0.410831000 \\ \mathrm{H} & 0.072402000 & 2.317738000 & 0.297932000 \\ \mathrm{~S} & 0.221648000 & 0.147809000 & 1.272246000 \\ \mathrm{C} & 1.908334000 & -0.470408000 & 0.898453000 \\ \mathrm{H} & 2.529349000 & -0.106190000 & 1.714435000 \\ \mathrm{H} & 1.898945000 & -1.557457000 & 0.945313000 \\ \mathrm{C} & -0.795525000 & -0.887329000 & 0.129317000 \\ \mathrm{H} & -0.473334000 & -1.919608000 & 0.250182000 \\ \mathrm{H} & -0.633103000 & -0.576085000 & -0.899084000 \\ \mathrm{C} & -2.259229000 & -0.736250000 & 0.496112000 \\ \mathrm{H} & -2.624557000 & 0.270355000 & 0.316250000 \\ \mathrm{H} & -2.454465000 & -1.040729000 & 1.519094000 \\ \mathrm{C} & 2.438453000 & 0.020642000 & -0.434298000 \\ \mathrm{H} & 1.851007000 & -0.337741000 & -1.272558000 \\ \mathrm{Cl} & -3.258178000 & -1.832009000 & -0.559918000\end{array}$




$\begin{array}{lrrr}\mathrm{H} & 2.502603000 & 1.102634000 & -0.473126000 \\ \mathrm{Cl} & 4.127906000 & -0.606687000 & -0.704701000\end{array}$

$\underline{\mathrm{G}}_{2}-$ Structure of A2-7

$\begin{array}{lrrr}\mathrm{O} & -3.941995000 & -1.246153000 & -0.409622000 \\ \mathrm{H} & -3.355422000 & -2.009838000 & -0.544349000 \\ \mathrm{H} & -4.599796000 & -1.288940000 & -1.107512000 \\ \mathrm{O} & -1.831998000 & -3.140680000 & -0.518826000 \\ \mathrm{H} & -1.208940000 & -2.530511000 & -0.089573000 \\ \mathrm{H} & -1.890738000 & -3.903321000 & 0.063446000 \\ \mathrm{~S} & 0.199655000 & -0.882749000 & 0.985800000 \\ \mathrm{C} & 1.602669000 & -0.650384000 & -0.177925000 \\ \mathrm{H} & 1.862867000 & -1.648151000 & -0.524500000 \\ \mathrm{H} & 1.274778000 & -0.076100000 & -1.040612000 \\ \mathrm{C} & -0.599980000 & 0.774411000 & 0.979792000 \\ \mathrm{H} & 0.169734000 & 1.533032000 & 1.101050000 \\ \mathrm{H} & -1.220370000 & 0.787361000 & 1.873327000 \\ \mathrm{C} & -1.439687000 & 1.023332000 & -0.257675000 \\ \mathrm{H} & -2.302838000 & 0.366968000 & -0.312522000 \\ \mathrm{H} & -0.859971000 & 0.953548000 & -1.172277000 \\ \mathrm{C} & 2.787707000 & 0.002518000 & 0.505724000 \\ \mathrm{H} & 2.560262000 & 0.999574000 & 0.867610000 \\ \mathrm{Cl} & -2.089126000 & 2.728205000 & -0.216939000 \\ \mathrm{H} & 3.163480000 & -0.602526000 & 1.323491000 \\ \mathrm{Cl} & 4.165606000 & 0.197576000 & -0.670255000\end{array}$




\section{$\underline{\mathrm{G}}_{3-}$-Structure of A3-1}

$\begin{array}{lrrr}\mathrm{S} & 0.445010000 & -2.395799000 & 0.482035000 \\ \mathrm{C} & 1.597061000 & -1.342324000 & -0.450161000 \\ \mathrm{H} & 2.315523000 & -2.013684000 & -0.915761000 \\ \mathrm{H} & 1.058019000 & -0.814289000 & -1.231821000 \\ \mathrm{C} & 2.290341000 & -0.369036000 & 0.476060000 \\ \mathrm{H} & 2.955020000 & -0.877607000 & 1.166666000 \\ \mathrm{H} & 1.586417000 & 0.245738000 & 1.027817000 \\ \mathrm{C} & -0.970196000 & -1.266728000 & 0.672629000 \\ \mathrm{H} & -1.569821000 & -1.691671000 & 1.475279000 \\ \mathrm{H} & -0.630086000 & -0.280408000 & 0.987422000 \\ \mathrm{C} & -1.776607000 & -1.191320000 & -0.604754000 \\ \mathrm{H} & -1.252680000 & -0.648750000 & -1.384699000 \\ \mathrm{H} & -2.069163000 & -2.176119000 & -0.951879000 \\ \mathrm{Cl} & -3.325218000 & -0.293694000 & -0.323349000 \\ \mathrm{Cl} & 3.320950000 & 0.775865000 & -0.475688000 \\ \mathrm{O} & 0.224424000 & 2.057609000 & 1.547670000 \\ \mathrm{H} & 0.676218000 & 2.744746000 & 2.040212000 \\ \mathrm{H} & -0.653742000 & 2.410835000 & 1.307270000 \\ \mathrm{O} & 0.133537000 & 1.504418000 & -1.183153000 \\ \mathrm{H} & 0.467200000 & 1.723699000 & -0.297472000 \\ \mathrm{H} & 0.871320000 & 1.669269000 & -1.775381000 \\ \mathrm{O} & -2.032759000 & 2.728294000 & 0.167629000 \\ \mathrm{H} & -2.711691000 & 2.049553000 & 0.230668000 \\ \mathrm{H} & -1.535343000 & 2.488437000 & -0.627072000 \\ & & \end{array}$




\section{$\underline{\mathrm{G}}_{3}-$ Structure of A3-2}

$\begin{array}{lrrr}\mathrm{S} & -0.627434000 & -2.380608000 & 0.509160000 \\ \mathrm{C} & 0.776713000 & -1.726715000 & -0.444559000 \\ \mathrm{H} & 1.273096000 & -2.598420000 & -0.866280000 \\ \mathrm{H} & 0.424987000 & -1.099005000 & -1.260224000 \\ \mathrm{C} & 1.728929000 & -0.952701000 & 0.438873000 \\ \mathrm{H} & 2.097338000 & -1.557402000 & 1.260666000 \\ \mathrm{H} & 1.287400000 & -0.035736000 & 0.819553000 \\ \mathrm{C} & -1.625189000 & -0.873957000 & 0.708269000 \\ \mathrm{H} & -2.315546000 & -1.074580000 & 1.525264000 \\ \mathrm{H} & -0.987866000 & -0.044927000 & 1.005863000 \\ \mathrm{C} & -2.382265000 & -0.564953000 & -0.563638000 \\ \mathrm{H} & -1.715193000 & -0.464040000 & -1.412312000 \\ \mathrm{H} & -3.142601000 & -1.310051000 & -0.772108000 \\ \mathrm{Cl} & -3.239106000 & 1.021306000 & -0.412647000 \\ \mathrm{Cl} & 3.183631000 & -0.461269000 & -0.522360000 \\ \mathrm{O} & 2.274687000 & 2.618131000 & 0.149146000 \\ \mathrm{H} & 3.035328000 & 2.031240000 & 0.174311000 \\ \mathrm{H} & 1.738922000 & 2.288649000 & -0.588048000 \\ \mathrm{O} & 0.062670000 & 1.989396000 & 1.623504000 \\ \mathrm{H} & 0.927788000 & 2.364244000 & 1.376663000 \\ \mathrm{H} & -0.372868000 & 2.624021000 & 2.194172000 \\ \mathrm{O} & 0.000174000 & 1.467387000 & -1.099126000 \\ \mathrm{H} & -0.631270000 & 1.847810000 & -1.714489000 \\ \mathrm{H} & -0.326863000 & 1.707923000 & -0.216343000 \\ & & & \end{array}$


G-ㅡㄴ Structure of A3-3

\begin{tabular}{|c|c|c|c|}
\hline & 0.131169000 & 5000 & 0.1 \\
\hline & -1.2002 & 8000 & 0000 \\
\hline & -1.9015 & 60000 & 2000 \\
\hline & -0.82 & 2000 & 4000 \\
\hline & 1.380358000 & 21000 & 0000 \\
\hline & 0.915072000 & -0.675349000 & 5000 \\
\hline & 2.077396000 & -1.98 & 4000 \\
\hline & 2.091866000 & 1000 & 3000 \\
\hline & 2.683926000 & -1.214097000 & 7000 \\
\hline & 1.424805000 & 6000 & 000 \\
\hline & -1.880276000 & -0.782827000 & 000 \\
\hline & -1.241760000 & -0.127881000 & 760000 \\
\hline & 3.269 & 0.6 & 1000 \\
\hline & -2.235909000 & -1.602995000 & 7416000 \\
\hline & -3.345945000 & 0.196013000 & -0.281477000 \\
\hline & -1.167386000 & 2.614666000 & 0.277699000 \\
\hline & -0.6 & 000 & 4000 \\
\hline & -1.99 & 000 & 9000 \\
\hline & 0.60 & 9000 & 1000 \\
\hline & 0.728616000 & 2000 & 56014000 \\
\hline & 1.489869000 & 2000 & -1.370974000 \\
\hline & 0.112260000 & 1.313883000 & 2.338423000 \\
\hline & -0.356137000 & 1.893961000 & 1.711552000 \\
\hline & 0.266154000 & 1.834170000 & 3.127367000 \\
\hline
\end{tabular}




\section{$\underline{\mathrm{G}}_{3}$ - Structure of A3-4}

$\begin{array}{crrr}\mathrm{O} & 0.048944000 & 1.908587000 & 1.842197000 \\ \mathrm{H} & -0.715964000 & 2.315914000 & 1.415902000 \\ \mathrm{H} & 0.083096000 & 2.269297000 & 2.729200000 \\ \mathrm{O} & -2.071488000 & 2.618379000 & 0.098032000 \\ \mathrm{H} & -2.664642000 & 3.323631000 & -0.166528000 \\ \mathrm{H} & -2.547411000 & 1.785977000 & -0.037522000 \\ \mathrm{~S} & 0.585406000 & -2.350736000 & 0.532519000 \\ \mathrm{C} & -0.884317000 & -1.288650000 & 0.681601000 \\ \mathrm{H} & -1.470837000 & -1.716129000 & 1.492701000 \\ \mathrm{H} & -0.590131000 & -0.278126000 & 0.961384000 \\ \mathrm{C} & -1.673465000 & -1.294654000 & -0.606327000 \\ \mathrm{H} & -1.978699000 & -2.294525000 & -0.894593000 \\ \mathrm{H} & -1.144299000 & -0.797213000 & -1.411368000 \\ \mathrm{C} & 1.686625000 & -1.268024000 & -0.428330000 \\ \mathrm{H} & 2.472690000 & -1.912189000 & -0.817119000 \\ \mathrm{H} & 1.141649000 & -0.841558000 & -1.266110000 \\ \mathrm{C} & 2.262716000 & -0.180694000 & 0.449754000 \\ \mathrm{H} & 1.495474000 & 0.435680000 & 0.907563000 \\ \mathrm{H} & 2.924577000 & -0.585718000 & 1.208032000 \\ \mathrm{Cl} & 3.266353000 & 0.950425000 & -0.551222000 \\ \mathrm{Cl} & -3.224300000 & -0.363605000 & -0.393260000 \\ \mathrm{O} & 0.039163000 & 1.290428000 & -1.315602000 \\ \mathrm{H} & 0.946371000 & 1.596438000 & -1.215447000 \\ \mathrm{H} & -0.515586000 & 1.987342000 & -0.947398000\end{array}$




\section{$\underline{\mathrm{G}}_{3-}$-Structure of A3-5}

$\begin{array}{lrrr}\mathrm{S} & -1.748621000 & -2.138875000 & 0.078895000 \\ \mathrm{C} & -2.213150000 & -0.488215000 & 0.686593000 \\ \mathrm{H} & -2.993030000 & -0.648058000 & 1.428299000 \\ \mathrm{H} & -1.360798000 & -0.022093000 & 1.173380000 \\ \mathrm{C} & -2.718151000 & 0.380390000 & -0.442904000 \\ \mathrm{H} & -3.646616000 & 0.004488000 & -0.859332000 \\ \mathrm{H} & -1.973685000 & 0.499375000 & -1.222068000 \\ \mathrm{C} & -0.123912000 & -1.776648000 & -0.654220000 \\ \mathrm{H} & 0.106404000 & -2.623916000 & -1.297098000 \\ \mathrm{H} & -0.174731000 & -0.874400000 & -1.258345000 \\ \mathrm{C} & 0.924475000 & -1.621735000 & 0.422850000 \\ \mathrm{H} & 0.731864000 & -0.751908000 & 1.040660000 \\ \mathrm{H} & 1.028045000 & -2.518860000 & 1.023387000 \\ \mathrm{Cl} & 2.545025000 & -1.333964000 & -0.342918000 \\ \mathrm{Cl} & -3.061924000 & 2.046537000 & 0.170176000 \\ \mathrm{O} & 0.191219000 & 1.339620000 & -0.134835000 \\ \mathrm{H} & 1.017051000 & 1.762389000 & -0.429987000 \\ \mathrm{H} & -0.421443000 & 2.060516000 & 0.035882000 \\ \mathrm{O} & 2.615982000 & 2.526117000 & -0.843651000 \\ \mathrm{H} & 3.226592000 & 2.153881000 & -0.186362000 \\ \mathrm{H} & 2.982209000 & 2.298456000 & -1.699435000 \\ \mathrm{O} & 4.111898000 & 1.150506000 & 1.088106000 \\ \mathrm{H} & 3.999650000 & 1.376163000 & 2.013117000 \\ \mathrm{H} & 3.675345000 & 0.299533000 & 0.961320000\end{array}$




\section{$\underline{\mathrm{G}}_{3}-$ Structure of A3-6}

$\begin{array}{lccc}\mathrm{S} & -0.071294000 & -2.404875000 & -0.098202000 \\ \mathrm{C} & 1.132833000 & -1.205524000 & -0.752400000 \\ \mathrm{H} & 1.618921000 & -1.677502000 & -1.603575000 \\ \mathrm{H} & 0.605611000 & -0.320425000 & -1.110472000 \\ \mathrm{C} & -1.286672000 & -1.253972000 & 0.615808000 \\ \mathrm{H} & -0.769828000 & -0.420058000 & 1.088890000 \\ \mathrm{H} & -1.821128000 & -1.798367000 & 1.391361000 \\ \mathrm{C} & -2.234050000 & -0.798435000 & -0.470883000 \\ \mathrm{H} & -2.866019000 & -1.605691000 & -0.825086000 \\ \mathrm{H} & -1.710097000 & -0.347195000 & -1.307794000 \\ \mathrm{C} & 2.145987000 & -0.847173000 & 0.311878000 \\ \mathrm{H} & 1.695962000 & -0.338311000 & 1.159870000 \\ \mathrm{Cl} & -3.355557000 & 0.483736000 & 0.158215000 \\ \mathrm{H} & 2.695932000 & -1.720509000 & 0.645915000 \\ \mathrm{Cl} & 3.387271000 & 0.284384000 & -0.376152000 \\ \mathrm{O} & 1.082911000 & 2.589026000 & 0.114374000 \\ \mathrm{H} & 0.386912000 & 2.445606000 & -0.543841000 \\ \mathrm{H} & 1.870299000 & 2.147727000 & -0.220325000 \\ \mathrm{O} & -1.124387000 & 2.042048000 & -1.569622000 \\ \mathrm{H} & -1.376248000 & 2.669718000 & -2.249945000 \\ \mathrm{H} & -1.899222000 & 1.937708000 & -1.002261000 \\ \mathrm{O} & 0.380695000 & 1.089249000 & 2.312159000 \\ \mathrm{H} & 0.291279000 & 1.567095000 & 3.137108000 \\ \mathrm{H} & 0.616839000 & 1.748220000 & 1.634636000\end{array}$




\section{$\underline{\mathrm{G}}_{3}-$ Structure of A3-7}

$\begin{array}{lrll}\mathrm{S} & 0.614117000 & -1.363817000 & 1.008023000 \\ \mathrm{C} & 1.948789000 & -0.984782000 & -0.168135000 \\ \mathrm{H} & 2.458426000 & -1.933588000 & -0.325313000 \\ \mathrm{H} & 1.538323000 & -0.666064000 & -1.123482000 \\ \mathrm{C} & 2.911165000 & 0.042289000 & 0.385675000 \\ \mathrm{H} & 3.373398000 & -0.303625000 & 1.304718000 \\ \mathrm{H} & 2.430767000 & 1.001127000 & 0.560460000 \\ \mathrm{C} & -0.213813000 & 0.251293000 & 1.189355000 \\ \mathrm{H} & -1.119568000 & 0.027417000 & 1.751722000 \\ \mathrm{H} & 0.401970000 & 0.919279000 & 1.787917000 \\ \mathrm{C} & -0.578136000 & 0.886792000 & -0.133410000 \\ \mathrm{H} & 0.295771000 & 1.145199000 & -0.724054000 \\ \mathrm{H} & -1.252746000 & 0.270398000 & -0.720339000 \\ \mathrm{Cl} & -1.426809000 & 2.449115000 & 0.170290000 \\ \mathrm{Cl} & 4.231785000 & 0.350065000 & -0.794257000 \\ \mathrm{O} & -3.616515000 & 0.027667000 & 0.673014000 \\ \mathrm{H} & -3.444403000 & 0.907550000 & 1.015790000 \\ \mathrm{H} & -3.636739000 & 0.129507000 & -0.291517000 \\ \mathrm{O} & -2.366630000 & -2.365563000 & -0.191725000 \\ \mathrm{H} & -2.766735000 & -1.747708000 & 0.437517000 \\ \mathrm{H} & -1.425683000 & -2.364905000 & 0.019832000 \\ \mathrm{O} & -3.059286000 & -0.458153000 & -2.033418000 \\ \mathrm{H} & -3.611766000 & -0.637057000 & -2.795274000 \\ \mathrm{H} & -2.820065000 & -1.317678000 & -1.642770000\end{array}$




\section{$\underline{\mathrm{G}}_{3}-$ Structure of A3-8}
S $\quad 0.399140000 \quad-0.444397000$
1.771267000
$\begin{array}{llll}\text { C } & -1.020834000 & -0.240349000 & 0.648358000\end{array}$
$\mathrm{H} \quad-1.644486000 \quad 0.543860000 \quad 1.075367000$
H $\quad-0.662618000 \quad 0.114743000 \quad-0.316048000$
$\begin{array}{llll}\text { C } & -1.783493000 & -1.541410000 & 0.539893000\end{array}$
$\mathrm{H} \quad-2.202374000 \quad-1.847293000 \quad 1.492503000$
$\mathrm{H} \quad-1.173386000 \quad-2.342689000 \quad 0.135254000$
$\begin{array}{llll}\text { C } & 1.632952000 & -1.139972000 & 0.624824000\end{array}$
$\begin{array}{llll}\mathrm{H} & 2.384296000 & -1.617803000 & 1.250362000\end{array}$
$\mathrm{H} \quad 1.163177000 \quad-1.914327000 \quad 0.020921000$
$\begin{array}{llll}\text { C } & 2.260513000 & -0.074703000 & -0.248715000\end{array}$
H $\quad 1.537211000 \quad 0.431937000 \quad-0.881278000$
$\mathrm{H} \quad 2.792826000 \quad 0.660175000 \quad 0.347249000$
$\mathrm{Cl} \quad 3.476444000 \quad-0.840024000 \quad-1.334821000$
$\mathrm{Cl} \quad-3.181174000 \quad-1.366950000 \quad-0.596380000$
$\begin{array}{llll}\text { O } & -2.825095000 & 1.932817000 & -0.983622000\end{array}$
$\mathrm{H} \quad-3.463892000 \quad 2.415016000 \quad-1.511793000$
$\mathrm{H} \quad-3.080421000 \quad 1.005181000 \quad-1.039826000$
$\begin{array}{llll}\text { O } & 0.051710000 & 2.124444000 & -1.294100000\end{array}$
$\mathrm{H} \quad 0.355890000 \quad 2.588248000 \quad-0.503931000$
$\mathrm{H} \quad-0.909484000 \quad 2.213497000 \quad-1.283968000$
$\begin{array}{llll}\text { O } & 0.848900000 & 2.832350000 & 1.370076000\end{array}$
$\mathrm{H} \quad 0.858154000 \quad 1.923922000 \quad 1.700625000$
$\begin{array}{llll}\mathrm{H} & 1.500347000 & 3.316163000 & 1.879721000\end{array}$ 


\section{$\underline{\mathrm{G}}_{3}-$ Structure of A3-9}

$\begin{array}{lrrr}\mathrm{S} & -0.990614000 & -1.208979000 & 0.699718000 \\ \mathrm{C} & -0.162870000 & 0.107016000 & -0.243009000 \\ \mathrm{H} & 0.726292000 & -0.342016000 & -0.678937000 \\ \mathrm{H} & -0.810827000 & 0.453150000 & -1.045820000 \\ \mathrm{C} & 0.234483000 & 1.241976000 & 0.673451000 \\ \mathrm{H} & 0.975644000 & 0.916169000 & 1.394795000 \\ \mathrm{H} & -0.615080000 & 1.687716000 & 1.180943000 \\ \mathrm{C} & -2.679218000 & -0.537421000 & 0.778765000 \\ \mathrm{H} & -3.161810000 & -1.015867000 & 1.628335000 \\ \mathrm{H} & -2.646447000 & 0.532733000 & 0.974994000 \\ \mathrm{C} & -3.443230000 & -0.832738000 & -0.493612000 \\ \mathrm{H} & -2.977564000 & -0.381813000 & -1.364428000 \\ \mathrm{H} & -3.546880000 & -1.900772000 & -0.652912000 \\ \mathrm{Cl} & -5.107909000 & -0.152180000 & -0.393459000 \\ \mathrm{Cl} & 0.996415000 & 2.579780000 & -0.280636000 \\ \mathrm{O} & 4.757497000 & -1.686071000 & -1.448699000 \\ \mathrm{H} & 5.099400000 & -1.460192000 & -2.314466000 \\ \mathrm{H} & 4.021561000 & -1.077483000 & -1.276428000 \\ \mathrm{O} & 5.532525000 & -0.643893000 & 1.013666000 \\ \mathrm{H} & 5.560427000 & -1.170843000 & 0.198840000 \\ \mathrm{H} & 5.679515000 & -1.254876000 & 1.736747000 \\ \mathrm{O} & 3.079033000 & 0.098916000 & -0.074833000 \\ \mathrm{H} & 2.953927000 & 1.034710000 & -0.258437000 \\ \mathrm{H} & 3.839633000 & 0.029234000 & 0.526276000\end{array}$




\subsubsection{Coordinates of the complexes of SM using B3LYP-D3/6-311++G(2d,2p)}

$\underline{\mathrm{G}_{0}-\text { structure of } \mathbf{A}}$

S $\quad 0.000000000 \quad 0.000000000 \quad 1.750414000$

$\begin{array}{llll}\text { C } & 0.565724000 & 1.298456000 & 0.583099000\end{array}$

$\mathrm{H} \quad 1.244259000 \quad 1.933063000 \quad 1.149099000$

H $\quad 1.134914000 \quad 0.848186000 \quad-0.226992000$

C $\quad-0.598612000 \quad 2.108300000 \quad 0.048191000$

$\begin{array}{llll}\mathrm{H} & -1.119160000 & 2.635268000 & 0.840838000\end{array}$

$\mathrm{H} \quad-1.305599000 \quad 1.498974000 \quad-0.505440000$

C $\quad-0.565724000 \quad-1.298456000 \quad 0.583099000$

H $\quad-1.244259000 \quad-1.933063000 \quad 1.149099000$

$\mathrm{H} \quad-1.134914000 \quad-0.848186000 \quad-0.226992000$

C $\quad 0.598612000 \quad-2.108300000 \quad 0.048191000$

$\mathrm{H} \quad 1.305599000 \quad-1.498974000 \quad-0.505440000$

H $\quad 1.119160000 \quad-2.635268000 \quad 0.840838000$

Cl $\quad 0.000000000 \quad-3.376850000 \quad-1.120503000$

$\mathrm{Cl} \quad 0.000000000 \quad 3.376850000 \quad-1.120503000$

\section{$\underline{\mathrm{G}_{1}-\text { Structure of A1-1 }}$}

$\begin{array}{llll}\mathrm{S} & 0.000106000 & 2.086869000 & 0.000146000 \\ \mathrm{C} & -1.284461000 & 0.917054000 & -0.595435000 \\ \mathrm{H} & -1.938511000 & 1.500009000 & -1.240410000 \\ \mathrm{H} & -0.821107000 & 0.136690000 & -1.190663000 \\ \mathrm{C} & -2.058136000 & 0.314426000 & 0.558446000 \\ \mathrm{H} & -2.654681000 & 1.051454000 & 1.085188000 \\ \mathrm{H} & -1.412121000 & -0.213024000 & 1.249170000\end{array}$




$\begin{array}{llll}\mathrm{C} & 1.284610000 & 0.916849000 & 0.595477000 \\ \mathrm{H} & 1.938759000 & 1.499664000 & 1.240478000 \\ \mathrm{H} & 0.821223000 & 0.136431000 & 1.190589000 \\ \mathrm{C} & 2.058115000 & 0.314317000 & -0.558551000 \\ \mathrm{H} & 1.411939000 & -0.213065000 & -1.249184000 \\ \mathrm{H} & 2.654543000 & 1.051403000 & -1.085347000 \\ \mathrm{Cl} & 3.232511000 & -0.951125000 & 0.063406000 \\ \mathrm{Cl} & -3.232426000 & -0.951013000 & -0.063857000 \\ \mathrm{O} & -0.000242000 & -1.963467000 & 0.000912000 \\ \mathrm{H} & 0.766320000 & -2.536909000 & 0.094628000 \\ \mathrm{H} & -0.768347000 & -2.534332000 & -0.096038000\end{array}$

$\underline{\mathrm{G}}_{1}-$ Structure of A1-2

$\begin{array}{lrrr}\mathrm{S} & -0.259445000 & -1.567887000 & 0.258023000 \\ \mathrm{C} & -1.722911000 & -0.534462000 & 0.658252000 \\ \mathrm{H} & -2.290892000 & -1.090323000 & 1.401123000 \\ \mathrm{H} & -1.404860000 & 0.398894000 & 1.117519000 \\ \mathrm{C} & -2.566644000 & -0.276887000 & -0.573895000 \\ \mathrm{H} & -2.964703000 & -1.197463000 & -0.987382000 \\ \mathrm{H} & -2.020238000 & 0.258581000 & -1.343497000 \\ \mathrm{C} & 0.885616000 & -0.305046000 & -0.421162000 \\ \mathrm{H} & 1.612921000 & -0.853024000 & -1.014338000 \\ \mathrm{H} & 0.344260000 & 0.373914000 & -1.076023000 \\ \mathrm{C} & 1.581434000 & 0.440055000 & 0.697860000 \\ \mathrm{H} & 0.892179000 & 0.970126000 & 1.346416000 \\ \mathrm{H} & 2.215490000 & -0.219667000 & 1.278672000 \\ \mathrm{Cl} & 2.690257000 & 1.734801000 & 0.007236000\end{array}$




$\begin{array}{lrrr}\mathrm{Cl} & -4.003121000 & 0.770605000 & -0.151441000 \\ \mathrm{O} & 4.002125000 & -1.262058000 & -0.430938000 \\ \mathrm{H} & 4.846552000 & -1.679226000 & -0.618569000 \\ \mathrm{H} & 4.157119000 & -0.313013000 & -0.499623000\end{array}$

\section{$\underline{\mathrm{G}_{1}-\text { Structure of A1-3 }}$}

$\begin{array}{lrrr}\mathrm{S} & -0.043855000 & 1.164977000 & 1.077367000 \\ \mathrm{C} & -1.372227000 & 0.667274000 & -0.088344000 \\ \mathrm{H} & -1.907908000 & 1.584477000 & -0.323971000 \\ \mathrm{H} & -0.937378000 & 0.294256000 & -1.011998000 \\ \mathrm{C} & -2.308146000 & -0.349766000 & 0.533481000 \\ \mathrm{H} & -2.800082000 & 0.044499000 & 1.416496000 \\ \mathrm{H} & -1.803610000 & -1.277355000 & 0.783675000 \\ \mathrm{C} & 1.088156000 & -0.282681000 & 1.004064000 \\ \mathrm{H} & 1.757037000 & -0.158479000 & 1.853206000 \\ \mathrm{H} & 0.518513000 & -1.194755000 & 1.165777000 \\ \mathrm{C} & 1.877674000 & -0.351809000 & -0.289247000 \\ \mathrm{H} & 1.240017000 & -0.458340000 & -1.160430000 \\ \mathrm{H} & 2.523451000 & 0.507996000 & -0.425672000 \\ \mathrm{Cl} & 2.947489000 & -1.829213000 & -0.265954000 \\ \mathrm{Cl} & -3.623274000 & -0.794288000 & -0.647987000 \\ \mathrm{O} & 1.835057000 & 2.777366000 & -1.172075000 \\ \mathrm{H} & 1.983789000 & 3.725519000 & -1.132099000 \\ \mathrm{H} & 1.222987000 & 2.575038000 & -0.448977000\end{array}$

$\underline{\mathrm{G}_{2}-\text { Structure of A2-1 }}$

$\begin{array}{llll}\mathrm{S} & 0.714554000 & -2.254911000 & 0.009096000\end{array}$ 


$\begin{array}{lrrr}\mathrm{C} & 1.742725000 & -0.856500000 & -0.590194000 \\ \mathrm{H} & 2.482725000 & -1.301112000 & -1.252342000 \\ \mathrm{H} & 1.129589000 & -0.169643000 & -1.164274000 \\ \mathrm{C} & -0.797167000 & -1.392002000 & 0.594738000 \\ \mathrm{H} & -0.527541000 & -0.515076000 & 1.175645000 \\ \mathrm{H} & -1.297736000 & -2.106253000 & 1.245172000 \\ \mathrm{C} & -1.686156000 & -1.006718000 & -0.567192000 \\ \mathrm{H} & -1.951728000 & -1.856334000 & -1.186158000 \\ \mathrm{H} & -1.252800000 & -0.212136000 & -1.162798000 \\ \mathrm{C} & 2.409535000 & -0.138262000 & 0.563475000 \\ \mathrm{H} & 1.685705000 & 0.282987000 & 1.250456000 \\ \mathrm{Cl} & -3.290588000 & -0.340567000 & 0.055731000 \\ \mathrm{H} & 3.113073000 & -0.772569000 & 1.091835000 \\ \mathrm{Cl} & 3.375764000 & 1.291696000 & -0.061684000 \\ \mathrm{O} & 0.003000000 & 1.725709000 & -0.005721000 \\ \mathrm{H} & -0.775433000 & 2.308286000 & -0.021308000 \\ \mathrm{H} & 0.766380000 & 2.275548000 & -0.204466000 \\ \mathrm{O} & -2.555336000 & 2.896292000 & -0.057231000 \\ \mathrm{H} & -2.881741000 & 3.340992000 & 0.729785000 \\ \mathrm{H} & -2.966300000 & 2.019583000 & -0.047223000\end{array}$

\section{$\underline{\mathrm{G}_{2}-\text { Structure of A2-3 }}$}

$\begin{array}{lrrr}\mathrm{O} & 0.008181000 & 1.959020000 & 1.524516000 \\ \mathrm{H} & -0.573011000 & 2.390879000 & 0.874038000 \\ \mathrm{H} & 0.047876000 & 2.550937000 & 2.278953000 \\ \mathrm{O} & -1.837027000 & 2.842793000 & -0.397783000\end{array}$




$\begin{array}{lrrr}\mathrm{H} & -1.588254000 & 3.080004000 & -1.294949000 \\ \mathrm{H} & -2.408746000 & 2.064921000 & -0.472514000 \\ \mathrm{~S} & 0.501924000 & -2.214134000 & 0.384443000 \\ \mathrm{C} & -0.978891000 & -1.165781000 & 0.662198000 \\ \mathrm{H} & -1.534853000 & -1.653666000 & 1.460251000 \\ \mathrm{H} & -0.683482000 & -0.179616000 & 1.011175000 \\ \mathrm{C} & 1.604066000 & -1.070474000 & -0.538525000 \\ \mathrm{H} & 1.030001000 & -0.524649000 & -1.283846000 \\ \mathrm{H} & 2.305552000 & -1.710694000 & -1.069696000 \\ \mathrm{C} & 2.336241000 & -0.118284000 & 0.385420000 \\ \mathrm{H} & 2.966905000 & -0.650074000 & 1.090204000 \\ \mathrm{H} & 1.664661000 & 0.553979000 & 0.907639000 \\ \mathrm{C} & -1.812527000 & -1.098884000 & -0.599558000 \\ \mathrm{H} & -1.291825000 & -0.615991000 & -1.419608000 \\ \mathrm{Cl} & 3.450189000 & 0.950292000 & -0.599818000 \\ \mathrm{H} & -2.162742000 & -2.075626000 & -0.914603000 \\ \mathrm{Cl} & -3.336391000 & -0.097246000 & -0.328959000\end{array}$

\section{$\underline{\mathrm{G}}_{2}$ - Structure of A2-4}

$\begin{array}{lrrr}\mathrm{S} & 0.075247000 & 0.224688000 & -1.710529000 \\ \mathrm{C} & -1.159709000 & -0.925698000 & -0.978903000 \\ \mathrm{H} & -1.760320000 & -1.273557000 & -1.816856000 \\ \mathrm{H} & -0.642167000 & -1.787712000 & -0.564712000 \\ \mathrm{C} & 1.368766000 & 0.274952000 & -0.404037000 \\ \mathrm{H} & 0.901737000 & 0.399543000 & 0.569789000 \\ \mathrm{H} & 1.946304000 & 1.174500000 & -0.608407000 \\ \mathrm{C} & 2.256600000 & -0.951203000 & -0.462076000\end{array}$




$\begin{array}{lrrr}\mathrm{H} & 2.771446000 & -1.035964000 & -1.413534000 \\ \mathrm{H} & 1.709800000 & -1.867447000 & -0.263402000 \\ \mathrm{C} & -2.028918000 & -0.256836000 & 0.066772000 \\ \mathrm{H} & -1.466878000 & 0.097915000 & 0.922911000 \\ \mathrm{Cl} & 3.547301000 & -0.845621000 & 0.822165000 \\ \mathrm{H} & -2.602703000 & 0.562658000 & -0.354326000 \\ \mathrm{Cl} & -3.244290000 & -1.460168000 & 0.705566000 \\ \mathrm{O} & -0.576670000 & 3.182850000 & -0.398584000 \\ \mathrm{H} & -0.545197000 & 2.392876000 & -0.966378000 \\ \mathrm{H} & -1.331299000 & 3.697246000 & -0.696863000 \\ \mathrm{O} & -0.379433000 & 1.786716000 & 2.047859000 \\ \mathrm{H} & -0.457561000 & 2.438960000 & 1.330994000 \\ \mathrm{H} & 0.150074000 & 2.200564000 & 2.733085000\end{array}$

$\underline{\mathrm{G}_{2}-\text { Structure of A2-5 }}$

$\begin{array}{lrrr}\mathrm{S} & -0.645216000 & -1.479694000 & 0.193483000 \\ \mathrm{C} & 0.436665000 & -0.130012000 & -0.419196000 \\ \mathrm{H} & 1.162657000 & -0.607766000 & -1.069659000 \\ \mathrm{H} & -0.149394000 & 0.579273000 & -0.999156000 \\ \mathrm{C} & -2.158749000 & -0.531410000 & 0.617782000 \\ \mathrm{H} & -1.886948000 & 0.398239000 & 1.113360000 \\ \mathrm{H} & -2.707102000 & -1.139046000 & 1.334417000 \\ \mathrm{C} & -3.003801000 & -0.267045000 & -0.611925000 \\ \mathrm{H} & -3.357173000 & -1.188779000 & -1.061842000 \\ \mathrm{H} & -2.476024000 & 0.319357000 & -1.357024000 \\ \mathrm{C} & 1.134093000 & 0.538836000 & 0.744001000 \\ \mathrm{H} & 0.445822000 & 0.977558000 & 1.458396000\end{array}$




$\begin{array}{llll}\mathrm{Cl} & -4.489964000 & 0.700374000 & -0.167321000 \\ \mathrm{H} & 1.817221000 & -0.144812000 & 1.233454000 \\ \mathrm{Cl} & 2.157965000 & 1.955765000 & 0.147064000 \\ \mathrm{O} & 2.958980000 & -1.981161000 & -0.026309000 \\ \mathrm{H} & 3.298658000 & -2.803251000 & 0.333519000 \\ \mathrm{H} & 3.732905000 & -1.414305000 & -0.186696000 \\ \mathrm{O} & 4.829544000 & 0.088073000 & -0.459408000 \\ \mathrm{H} & 4.183188000 & 0.790014000 & -0.293833000 \\ \mathrm{H} & 5.146197000 & 0.236762000 & -1.354512000\end{array}$

\section{$\underline{\mathrm{G}}_{2}-$ Structure of A2-6}

$\begin{array}{lrrr}\mathrm{O} & 2.973930000 & -1.973476000 & 0.049847000 \\ \mathrm{H} & 3.724285000 & -1.420041000 & -0.226850000 \\ \mathrm{H} & 3.233682000 & -2.881930000 & -0.116948000 \\ \mathrm{O} & 4.773331000 & 0.087189000 & -0.652058000 \\ \mathrm{H} & 4.110051000 & 0.778307000 & -0.508919000 \\ \mathrm{H} & 5.495401000 & 0.299785000 & -0.054487000 \\ \mathrm{~S} & -0.644931000 & -1.479750000 & 0.206692000 \\ \mathrm{C} & 0.440519000 & -0.133315000 & -0.406388000 \\ \mathrm{H} & 1.169820000 & -0.613494000 & -1.051444000 \\ \mathrm{H} & -0.142238000 & 0.574832000 & -0.990969000 \\ \mathrm{C} & -2.160451000 & -0.529903000 & 0.619680000 \\ \mathrm{H} & -1.890651000 & 0.400509000 & 1.114956000 \\ \mathrm{H} & -2.713001000 & -1.135797000 & 1.334568000 \\ \mathrm{C} & -2.998871000 & -0.267082000 & -0.614878000 \\ \mathrm{H} & -3.350187000 & -1.189327000 & -1.065353000 \\ \mathrm{H} & -2.467161000 & 0.318275000 & -1.357980000\end{array}$




$\begin{array}{lrrr}\mathrm{C} & 1.135246000 & 0.538957000 & 0.756534000 \\ \mathrm{H} & 0.445019000 & 0.980450000 & 1.467378000 \\ \mathrm{Cl} & -4.487056000 & 0.701204000 & -0.178966000 \\ \mathrm{H} & 1.816652000 & -0.141580000 & 1.252878000 \\ \mathrm{Cl} & 2.158261000 & 1.954347000 & 0.154501000\end{array}$

$\underline{\mathrm{G}}_{2}$ - Structure of A2-7

$\begin{array}{lrrr}\mathrm{S} & -0.232291000 & -1.302907000 & -0.710172000 \\ \mathrm{C} & -1.735934000 & -0.830220000 & 0.232080000 \\ \mathrm{H} & -2.218590000 & -1.773317000 & 0.480864000 \\ \mathrm{H} & -1.462157000 & -0.343936000 & 1.164645000 \\ \mathrm{C} & 0.682895000 & 0.284186000 & -0.863089000 \\ \mathrm{H} & 0.114402000 & 0.985709000 & -1.468907000 \\ \mathrm{H} & 1.587857000 & 0.006167000 & -1.399528000 \\ \mathrm{C} & 1.051527000 & 0.885608000 & 0.478474000 \\ \mathrm{H} & 1.610956000 & 0.192140000 & 1.095612000 \\ \mathrm{H} & 0.190742000 & 1.253823000 & 1.027268000 \\ \mathrm{C} & -2.665761000 & 0.039774000 & -0.588968000 \\ \mathrm{H} & -2.209939000 & 0.985490000 & -0.863606000 \\ \mathrm{Cl} & 2.128849000 & 2.337831000 & 0.227622000 \\ \mathrm{H} & -3.008636000 & -0.470452000 & -1.483079000 \\ \mathrm{Cl} & -4.149564000 & 0.460645000 & 0.382445000 \\ \mathrm{O} & 3.763749000 & -0.724035000 & -0.683626000 \\ \mathrm{H} & 4.010196000 & 0.128281000 & -0.313185000 \\ \mathrm{H} & 3.430364000 & -1.246724000 & 0.061949000 \\ \mathrm{O} & 2.252779000 & -2.199695000 & 1.292920000 \\ \mathrm{H} & 2.473182000 & -3.117134000 & 1.475523000\end{array}$




$\begin{array}{lrrr}\mathrm{H} & 1.421838000 & -2.213864000 & 0.788719000 \\ \mathrm{G} 2-\text { Structure of A2-8 } & & \\ \mathrm{O} & -2.023915000 & 2.997978000 & -0.779098000 \\ \mathrm{H} & -1.072736000 & 3.194731000 & -0.759922000 \\ \mathrm{H} & -2.316793000 & 3.193983000 & -1.671898000 \\ \mathrm{O} & 0.773466000 & 3.151320000 & -0.250704000 \\ \mathrm{H} & 1.026474000 & 3.911441000 & 0.280758000 \\ \mathrm{H} & 0.650986000 & 2.417175000 & 0.378223000 \\ \mathrm{~S} & 0.065778000 & 0.406260000 & 1.416988000 \\ \mathrm{C} & 1.472286000 & -0.666114000 & 0.915381000 \\ \mathrm{H} & 2.137046000 & -0.709845000 & 1.775338000 \\ \mathrm{H} & 1.105395000 & -1.672737000 & 0.727933000 \\ \mathrm{C} & 2.197249000 & -0.112261000 & -0.294954000 \\ \mathrm{H} & 2.639564000 & 0.857998000 & -0.096162000 \\ \mathrm{H} & 1.553247000 & -0.036681000 & -1.164761000 \\ \mathrm{C} & -1.204919000 & -0.067541000 & 0.173485000 \\ \mathrm{H} & -1.859427000 & 0.794156000 & 0.063244000 \\ \mathrm{H} & -0.721753000 & -0.232305000 & -0.786704000 \\ \mathrm{C} & -1.961214000 & -1.300074000 & 0.625414000 \\ \mathrm{H} & -1.311903000 & -2.158380000 & 0.768550000 \\ \mathrm{H} & -2.522588000 & -1.115981000 & 1.535605000 \\ \mathrm{Cl} & -3.172733000 & -1.789262000 & -0.646562000 \\ \mathrm{Cl} & 3.562334000 & -1.226160000 & -0.765177000\end{array}$

$\underline{\mathrm{G}_{3}-\text { Structure of A3-1 }}$
S $\quad-0.510861000 \quad-2.477359000 \quad-0.164144000$
$\begin{array}{llll}\text { C } & -1.640848000 & -1.217783000 & 0.545523000\end{array}$ 


$\begin{array}{lrrr}\mathrm{H} & -2.371776000 & -1.777311000 & 1.125700000 \\ \mathrm{H} & -1.093464000 & -0.568021000 & 1.221459000 \\ \mathrm{C} & 0.958127000 & -1.470876000 & -0.617104000 \\ \mathrm{H} & 0.661432000 & -0.594451000 & -1.188252000 \\ \mathrm{H} & 1.542930000 & -2.118523000 & -1.267318000 \\ \mathrm{C} & 1.759418000 & -1.084883000 & 0.609185000 \\ \mathrm{H} & 2.021666000 & -1.946385000 & 1.213116000 \\ \mathrm{H} & 1.257995000 & -0.335283000 & 1.212312000 \\ \mathrm{C} & -2.316409000 & -0.414621000 & -0.545119000 \\ \mathrm{H} & -1.604789000 & 0.122448000 & -1.160831000 \\ \mathrm{Cl} & 3.364131000 & -0.333977000 & 0.108700000 \\ \mathrm{H} & -2.962951000 & -1.027355000 & -1.163867000 \\ \mathrm{Cl} & -3.393888000 & 0.876042000 & 0.197728000 \\ \mathrm{O} & 0.011433000 & 1.826898000 & -1.511779000 \\ \mathrm{H} & 0.857881000 & 2.223945000 & -1.211787000 \\ \mathrm{H} & -0.397814000 & 2.470471000 & -2.095633000 \\ \mathrm{O} & 2.122685000 & 2.760027000 & -0.032576000 \\ \mathrm{H} & 1.547837000 & 2.655104000 & 0.740966000 \\ \mathrm{H} & 2.755205000 & 2.032948000 & 0.032510000 \\ \mathrm{O} & -0.212841000 & 1.744168000 & 1.282811000 \\ \mathrm{H} & -0.451773000 & 1.783953000 & 0.339299000 \\ \mathrm{H} & -1.014680000 & 1.981342000 & 1.756815000\end{array}$

$\underline{\mathrm{G}_{3}-\text { Structure of A3-2 }}$
S $\quad-0.504680000 \quad-2.437466000$
0.110561000
$\begin{array}{llll}\text { C } & 0.911427000 & -1.503231000 & -0.595119000\end{array}$
H $\quad 1.388402000 \quad-2.178126000 \quad-1.302453000$
$\mathrm{H} \quad 0.549822000 \quad-0.634225000 \quad-1.140726000$ 


$\begin{array}{lrrr}\mathrm{C} & 1.880837000 & -1.100083000 & 0.496795000 \\ \mathrm{H} & 2.241712000 & -1.955113000 & 1.057654000 \\ \mathrm{H} & 1.464707000 & -0.357257000 & 1.168778000 \\ \mathrm{C} & -1.609559000 & -1.068195000 & 0.635275000 \\ \mathrm{H} & -2.250406000 & -1.476116000 & 1.414023000 \\ \mathrm{H} & -1.016150000 & -0.268670000 & 1.069375000 \\ \mathrm{C} & -2.430378000 & -0.577609000 & -0.539071000 \\ \mathrm{H} & -1.804415000 & -0.223034000 & -1.349102000 \\ \mathrm{H} & -3.123549000 & -1.330450000 & -0.898339000 \\ \mathrm{Cl} & -3.451773000 & 0.866438000 & -0.044421000 \\ \mathrm{Cl} & 3.383577000 & -0.335191000 & -0.244457000 \\ \mathrm{O} & 2.119195000 & 2.765179000 & -0.108840000 \\ \mathrm{H} & 2.744833000 & 2.042744000 & -0.247566000 \\ \mathrm{H} & 1.448240000 & 2.648122000 & -0.800352000 \\ \mathrm{O} & 0.237846000 & 1.721468000 & 1.573265000 \\ \mathrm{H} & 1.012334000 & 2.195003000 & 1.198281000 \\ \mathrm{H} & -0.106643000 & 2.267072000 & 2.284401000 \\ \mathrm{O} & -0.376080000 & 1.847044000 & -1.163950000 \\ \mathrm{H} & -1.176922000 & 2.255561000 & -1.504367000 \\ \mathrm{H} & -0.499407000 & 1.807923000 & -0.198740000\end{array}$

$\underline{\mathrm{G}}_{3}-$ Structure of A3-3

$\begin{array}{lrrr}\mathrm{S} & -0.104132000 & -2.456942000 & -0.059011000 \\ \mathrm{C} & 1.138312000 & -1.286213000 & -0.741861000 \\ \mathrm{H} & 1.617627000 & -1.794352000 & -1.575646000 \\ \mathrm{H} & 0.630161000 & -0.406828000 & -1.132397000 \\ \mathrm{C} & -1.337007000 & -1.275147000 & 0.617276000 \\ \mathrm{H} & -0.825605000 & -0.448666000 & 1.104808000\end{array}$




$\begin{array}{rrrr}\mathrm{H} & -1.897408000 & -1.816607000 & 1.376337000 \\ \mathrm{C} & -2.247724000 & -0.808280000 & -0.498112000 \\ \mathrm{H} & -2.888970000 & -1.599670000 & -0.870448000 \\ \mathrm{H} & -1.700653000 & -0.359672000 & -1.319898000 \\ \mathrm{C} & 2.152874000 & -0.907056000 & 0.316370000 \\ \mathrm{H} & 1.708672000 & -0.385491000 & 1.157316000 \\ \mathrm{Cl} & -3.382539000 & 0.515405000 & 0.104832000 \\ \mathrm{H} & 2.719889000 & -1.764986000 & 0.661139000 \\ \mathrm{Cl} & 3.394440000 & 0.248795000 & -0.402420000 \\ \mathrm{O} & 1.156713000 & 2.719292000 & 0.176148000 \\ \mathrm{H} & 0.450496000 & 2.575912000 & -0.477155000 \\ \mathrm{H} & 1.924539000 & 2.236259000 & -0.153313000 \\ \mathrm{O} & -1.046497000 & 2.138174000 & -1.503477000 \\ \mathrm{H} & -1.323347000 & 2.772372000 & -2.170538000 \\ \mathrm{H} & -1.824288000 & 1.981745000 & -0.947589000 \\ \mathrm{O} & 0.355762000 & 1.100398000 & 2.259853000 \\ \mathrm{H} & 0.275086000 & 1.539251000 & 3.109228000 \\ \mathrm{H} & 0.631042000 & 1.787671000 & 1.619104000\end{array}$

G3-Structure of A3-4

$\begin{array}{lrrr}\mathrm{O} & -0.128564000 & 1.594324000 & 2.078469000 \\ \mathrm{H} & -0.731779000 & 2.129829000 & 1.540779000 \\ \mathrm{H} & -0.104253000 & 2.004804000 & 2.945688000 \\ \mathrm{O} & -1.863938000 & 2.625205000 & 0.082596000 \\ \mathrm{H} & -2.391425000 & 3.405914000 & -0.105209000 \\ \mathrm{H} & -2.447933000 & 1.857373000 & -0.040316000 \\ \mathrm{~S} & 0.544950000 & -2.448576000 & 0.298266000\end{array}$




$\begin{array}{lrrr}\mathrm{C} & -0.954556000 & -1.439435000 & 0.621482000 \\ \mathrm{H} & -1.569694000 & -2.062013000 & 1.268607000 \\ \mathrm{H} & -0.696010000 & -0.536143000 & 1.167786000 \\ \mathrm{C} & -1.683514000 & -1.122177000 & -0.665923000 \\ \mathrm{H} & -1.976224000 & -2.013780000 & -1.208975000 \\ \mathrm{H} & -1.129273000 & -0.442639000 & -1.303715000 \\ \mathrm{C} & 1.670464000 & -1.231441000 & -0.490563000 \\ \mathrm{H} & 2.464979000 & -1.828634000 & -0.933856000 \\ \mathrm{H} & 1.150584000 & -0.710487000 & -1.288093000 \\ \mathrm{C} & 2.229612000 & -0.253586000 & 0.521010000 \\ \mathrm{H} & 1.463539000 & 0.334864000 & 1.012322000 \\ \mathrm{H} & 2.856924000 & -0.742493000 & 1.258873000 \\ \mathrm{Cl} & 3.308608000 & 0.970462000 & -0.329157000 \\ \mathrm{Cl} & -3.272844000 & -0.246950000 & -0.292736000 \\ \mathrm{O} & 0.180869000 & 1.522894000 & -1.651626000 \\ \mathrm{H} & 1.087128000 & 1.735426000 & -1.402304000 \\ \mathrm{H} & -0.382718000 & 2.085942000 & -1.103216000\end{array}$

$\underline{\mathrm{G}}_{3}-$ Structure of A3-5
S $\quad 1.665230000 \quad-2.197815000 \quad-0.119731000$
$\begin{array}{llll}\text { C } & 2.230288000 & -0.544258000 & -0.680649000\end{array}$
H $\quad 3.012536000 \quad-0.737657000 \quad-1.411836000$
H $\quad 1.415502000 \quad-0.025942000 \quad-1.175716000$
$\begin{array}{llll}\text { C } & 2.763605000 & 0.272428000 & 0.476567000\end{array}$
$\mathrm{H} \quad 3.643973000 \quad-0.175201000 \quad 0.924904000$
$\mathrm{H} \quad 2.004847000 \quad 0.455023000 \quad 1.228047000$
$\begin{array}{llll}\text { C } & 0.032345000 & -1.814287000 & 0.626398000\end{array}$
$\begin{array}{llll}\mathrm{H} & -0.209723000 & -2.685766000 & 1.231425000\end{array}$ 


$\begin{array}{lrrr}\mathrm{H} & 0.104401000 & -0.945752000 & 1.274421000 \\ \mathrm{C} & -1.017927000 & -1.590059000 & -0.439690000 \\ \mathrm{H} & -0.871711000 & -0.648000000 & -0.954885000 \\ \mathrm{H} & -1.085325000 & -2.418480000 & -1.135894000 \\ \mathrm{Cl} & -2.681384000 & -1.470005000 & 0.348197000 \\ \mathrm{Cl} & 3.274433000 & 1.933727000 & -0.113037000 \\ \mathrm{O} & -0.116487000 & 1.465115000 & 0.138022000 \\ \mathrm{H} & -0.916378000 & 2.003622000 & 0.292923000 \\ \mathrm{H} & 0.577597000 & 2.100037000 & -0.065172000 \\ \mathrm{O} & -2.445231000 & 2.979491000 & 0.448171000 \\ \mathrm{H} & -3.138591000 & 2.441287000 & 0.020531000 \\ \mathrm{H} & -2.762745000 & 3.159901000 & 1.336310000 \\ \mathrm{O} & -4.233235000 & 1.208533000 & -0.746749000 \\ \mathrm{H} & -4.376155000 & 1.235865000 & -1.696499000 \\ \mathrm{H} & -3.813977000 & 0.354749000 & -0.561886000\end{array}$

\section{$\underline{G}_{3-}$ Structure of A3-6}

$\begin{array}{lrrr}\text { S } & 0.551766000 & -1.061492000 & -0.543803000 \\ \mathrm{C} & 2.239746000 & -0.371601000 & -0.755429000 \\ \mathrm{H} & 2.710392000 & -0.963107000 & -1.537660000 \\ \mathrm{H} & 2.168204000 & 0.653743000 & -1.112041000 \\ \mathrm{C} & 3.039022000 & -0.452234000 & 0.529229000 \\ \mathrm{H} & 3.182942000 & -1.479168000 & 0.848216000 \\ \mathrm{H} & 2.584325000 & 0.116039000 & 1.334273000 \\ \mathrm{C} & -0.302436000 & 0.348609000 & 0.266966000 \\ \mathrm{H} & -1.071539000 & -0.079227000 & 0.902730000 \\ \mathrm{H} & 0.400868000 & 0.884616000 & 0.900034000 \\ \mathrm{C} & -0.933869000 & 1.262174000 & -0.761928000\end{array}$




$\begin{array}{lrrr}\mathrm{H} & -0.200506000 & 1.741794000 & -1.402151000 \\ \mathrm{H} & -1.674353000 & 0.739208000 & -1.356711000 \\ \mathrm{Cl} & -1.822533000 & 2.622612000 & 0.091378000 \\ \mathrm{Cl} & 4.702338000 & 0.258628000 & 0.283334000 \\ \mathrm{O} & -3.549050000 & -0.171598000 & 1.363028000 \\ \mathrm{H} & -3.320235000 & 0.751569000 & 1.509142000 \\ \mathrm{H} & -3.699026000 & -0.247343000 & 0.404973000 \\ \mathrm{O} & -2.240165000 & -2.631476000 & 0.758067000 \\ \mathrm{H} & -2.554863000 & -1.909023000 & 1.326394000 \\ \mathrm{H} & -1.291394000 & -2.700661000 & 0.902238000 \\ \mathrm{O} & -3.272323000 & -1.067204000 & -1.294145000 \\ \mathrm{H} & -3.925130000 & -1.463930000 & -1.875766000 \\ \mathrm{H} & -2.857106000 & -1.801195000 & -0.801561000\end{array}$

$\underline{\mathrm{G}}_{3}-$ Structure of A3-7

$\begin{array}{lrrr}\mathrm{S} & 0.561198000 & -1.245465000 & 1.498655000 \\ \mathrm{C} & -0.965968000 & -0.602658000 & 0.703732000 \\ \mathrm{H} & -1.583559000 & -0.228064000 & 1.517517000 \\ \mathrm{H} & -0.706587000 & 0.239718000 & 0.067438000 \\ \mathrm{C} & -1.681713000 & -1.698824000 & -0.055124000 \\ \mathrm{H} & -1.985962000 & -2.516720000 & 0.589003000 \\ \mathrm{H} & -1.098237000 & -2.082622000 & -0.885312000 \\ \mathrm{C} & 1.736836000 & -1.259120000 & 0.084121000 \\ \mathrm{H} & 2.515033000 & -1.966587000 & 0.363145000 \\ \mathrm{H} & 1.231799000 & -1.655841000 & -0.793500000 \\ \mathrm{C} & 2.324578000 & 0.109627000 & -0.197800000 \\ \mathrm{H} & 1.577134000 & 0.842608000 & -0.483588000 \\ \mathrm{H} & 2.896391000 & 0.480303000 & 0.647332000\end{array}$




$\begin{array}{llll}\mathrm{Cl} & 3.496405000 & -0.017217000 & -1.592986000 \\ \mathrm{Cl} & -3.230784000 & -1.052401000 & -0.800863000 \\ \mathrm{O} & -2.763245000 & 2.326247000 & -1.084465000 \\ \mathrm{H} & -2.925557000 & 2.598418000 & -1.991836000 \\ \mathrm{H} & -2.955959000 & 1.379905000 & -1.059056000 \\ \mathrm{O} & 0.003222000 & 2.439440000 & -0.266504000 \\ \mathrm{H} & 0.036826000 & 2.506425000 & 0.698690000 \\ \mathrm{H} & -0.926058000 & 2.561623000 & -0.513125000 \\ \mathrm{O} & 0.309374000 & 1.901205000 & 2.566655000 \\ \mathrm{H} & 0.635243000 & 0.994691000 & 2.449440000 \\ \mathrm{H} & 0.917563000 & 2.327794000 & 3.175741000\end{array}$

$\underline{\mathrm{G}}_{3}$ - Structure of A3-8

$\begin{array}{lrrr}\mathrm{S} & 1.065803000 & -1.098300000 & -0.988202000 \\ \mathrm{C} & 0.132445000 & 0.082147000 & 0.061183000 \\ \mathrm{H} & -0.789470000 & -0.424196000 & 0.333682000 \\ \mathrm{H} & 0.692884000 & 0.295105000 & 0.968377000 \\ \mathrm{C} & -0.176930000 & 1.349262000 & -0.707676000 \\ \mathrm{H} & -0.848265000 & 1.157281000 & -1.536873000 \\ \mathrm{H} & 0.714005000 & 1.859379000 & -1.058027000 \\ \mathrm{C} & 2.770382000 & -0.435804000 & -0.825833000 \\ \mathrm{H} & 3.323366000 & -0.813111000 & -1.683362000 \\ \mathrm{H} & 2.755580000 & 0.649755000 & -0.894726000 \\ \mathrm{C} & 3.417206000 & -0.892129000 & 0.466356000 \\ \mathrm{H} & 2.879910000 & -0.544506000 & 1.342752000 \\ \mathrm{H} & 3.514759000 & -1.971774000 & 0.507002000 \\ \mathrm{Cl} & 5.105583000 & -0.208118000 & 0.605321000 \\ \mathrm{Cl} & -1.035869000 & 2.562090000 & 0.374237000\end{array}$




$\begin{array}{llll}\mathrm{O} & -4.562556000 & -1.788217000 & 1.505588000 \\ \mathrm{H} & -4.777631000 & -1.556749000 & 2.412277000 \\ \mathrm{H} & -3.876968000 & -1.158205000 & 1.216950000 \\ \mathrm{O} & -5.782127000 & -0.651437000 & -0.746465000 \\ \mathrm{H} & -5.647391000 & -1.204753000 & 0.044672000 \\ \mathrm{H} & -6.011904000 & -1.253736000 & -1.458213000 \\ \mathrm{O} & -3.156461000 & 0.053368000 & -0.083082000 \\ \mathrm{H} & -3.004488000 & 0.978059000 & 0.140916000 \\ \mathrm{H} & -4.011853000 & 0.012159000 & -0.549193000\end{array}$

$\underline{\mathrm{G}}_{3-}$ - Structure of A3-9

$\begin{array}{lrrr}\mathrm{S} & -0.990614000 & -1.208979000 & 0.699718000 \\ \mathrm{C} & -0.162870000 & 0.107016000 & -0.243009000 \\ \mathrm{H} & 0.726292000 & -0.342016000 & -0.678937000 \\ \mathrm{H} & -0.810827000 & 0.453150000 & -1.045820000 \\ \mathrm{C} & 0.234483000 & 1.241976000 & 0.673451000 \\ \mathrm{H} & 0.975644000 & 0.916169000 & 1.394795000 \\ \mathrm{H} & -0.615080000 & 1.687716000 & 1.180943000 \\ \mathrm{C} & -2.679218000 & -0.537421000 & 0.778765000 \\ \mathrm{H} & -3.161810000 & -1.015867000 & 1.628335000 \\ \mathrm{H} & -2.646447000 & 0.532733000 & 0.974994000 \\ \mathrm{C} & -3.443230000 & -0.832738000 & -0.493612000 \\ \mathrm{H} & -2.977564000 & -0.381813000 & -1.364428000 \\ \mathrm{H} & -3.546880000 & -1.900772000 & -0.652912000 \\ \mathrm{Cl} & -5.107909000 & -0.152180000 & -0.393459000 \\ \mathrm{Cl} & 0.996415000 & 2.579780000 & -0.280636000 \\ \mathrm{O} & 4.757497000 & -1.686071000 & -1.448699000 \\ \mathrm{H} & 5.099400000 & -1.460192000 & -2.314466000\end{array}$




$\begin{array}{lrrr}\mathrm{H} & 4.021561000 & -1.077483000 & -1.276428000 \\ \mathrm{O} & 5.532525000 & -0.643893000 & 1.013666000 \\ \mathrm{H} & 5.560427000 & -1.170843000 & 0.198840000 \\ \mathrm{H} & 5.679515000 & -1.254876000 & 1.736747000 \\ \mathrm{O} & 3.079033000 & 0.098916000 & -0.074833000 \\ \mathrm{H} & 2.953927000 & 1.034710000 & -0.258437000 \\ \mathrm{H} & 3.839633000 & 0.029234000 & 0.526276000\end{array}$

\subsubsection{Coordinates of the complexes of SM using MP2/6-311++G(2d,2p)}

$\underline{\mathrm{G}}_{0}-$ structure of $\mathbf{A}$

$\begin{array}{lrrr}\mathrm{S} & 0.000000000 & 0.000000000 & 1.745865000 \\ \mathrm{C} & 0.578959000 & 1.267968000 & 0.572357000 \\ \mathrm{H} & 1.284598000 & 1.889878000 & 1.114689000 \\ \mathrm{H} & 1.110784000 & 0.795137000 & -0.247745000 \\ \mathrm{C} & -0.582418000 & 2.094668000 & 0.072011000 \\ \mathrm{H} & -1.053891000 & 2.641573000 & 0.878135000 \\ \mathrm{H} & -1.321898000 & 1.493720000 & -0.441576000 \\ \mathrm{C} & -0.578959000 & -1.267968000 & 0.572357000 \\ \mathrm{H} & -1.284598000 & -1.889878000 & 1.114689000 \\ \mathrm{H} & -1.110784000 & -0.795137000 & -0.247745000 \\ \mathrm{C} & 0.582418000 & -2.094668000 & 0.072011000 \\ \mathrm{H} & 1.321898000 & -1.493720000 & -0.441576000 \\ \mathrm{H} & 1.053891000 & -2.641573000 & 0.878135000 \\ \mathrm{Cl} & 0.000000000 & -3.322737000 & -1.125684000 \\ \mathrm{Cl} & 0.000000000 & 3.322737000 & -1.125684000\end{array}$




\section{$\underline{\mathrm{G}}_{1}$ - Structure of A1-1}

$\begin{array}{lrrr}\mathrm{S} & 0.000000000 & 0.000000000 & 2.074229000 \\ \mathrm{C} & 0.601079000 & 1.257045000 & 0.899550000 \\ \mathrm{H} & 1.258324000 & 1.910359000 & 1.466811000 \\ \mathrm{H} & 1.182626000 & 0.786884000 & 0.114351000 \\ \mathrm{C} & -0.550024000 & 2.039387000 & 0.312294000 \\ \mathrm{H} & -1.065517000 & 2.618428000 & 1.068359000 \\ \mathrm{H} & -1.249176000 & 1.394936000 & -0.203046000 \\ \mathrm{C} & -0.601079000 & -1.257045000 & 0.899550000 \\ \mathrm{H} & -1.258324000 & -1.910359000 & 1.466811000 \\ \mathrm{H} & -1.182626000 & -0.786884000 & 0.114351000 \\ \mathrm{C} & 0.550024000 & -2.039387000 & 0.312294000 \\ \mathrm{H} & 1.249176000 & -1.394936000 & -0.203046000 \\ \mathrm{H} & 1.065517000 & -2.618428000 & 1.068359000 \\ \mathrm{Cl} & -0.060959000 & -3.211534000 & -0.924965000 \\ \mathrm{Cl} & 0.060959000 & 3.211534000 & -0.924965000 \\ \mathrm{O} & 0.000000000 & 0.000000000 & -2.001291000 \\ \mathrm{H} & -0.087795000 & -0.759969000 & -2.581809000 \\ \mathrm{H} & 0.087795000 & 0.759969000 & -2.581809000\end{array}$

$\underline{\mathrm{G}_{1}-\text { Structure of A1-2 }}$

$\begin{array}{lrrr}\mathrm{S} & -0.255351000 & -1.582405000 & 0.273370000 \\ \mathrm{C} & -1.694127000 & -0.535953000 & 0.664887000 \\ \mathrm{H} & -2.272786000 & -1.067422000 & 1.414809000 \\ \mathrm{H} & -1.365738000 & 0.399960000 & 1.108139000 \\ \mathrm{C} & -2.532855000 & -0.289150000 & -0.568349000 \\ \mathrm{H} & -2.937682000 & -1.215230000 & -0.956575000\end{array}$




$\begin{array}{lrrr}\mathrm{H} & -1.968925000 & 0.207479000 & -1.348488000 \\ \mathrm{C} & 0.858097000 & -0.314747000 & -0.411866000 \\ \mathrm{H} & 1.585235000 & -0.839035000 & -1.023164000 \\ \mathrm{H} & 0.299576000 & 0.365594000 & -1.048620000 \\ \mathrm{C} & 1.558058000 & 0.424246000 & 0.704453000 \\ \mathrm{H} & 0.863466000 & 0.922186000 & 1.369886000 \\ \mathrm{H} & 2.197414000 & -0.242414000 & 1.267865000 \\ \mathrm{Cl} & 2.622591000 & 1.725571000 & 0.024344000 \\ \mathrm{Cl} & -3.928960000 & 0.782909000 & -0.171099000 \\ \mathrm{O} & 3.975063000 & -1.210847000 & -0.476666000 \\ \mathrm{H} & 4.825694000 & -1.612803000 & -0.659099000 \\ \mathrm{H} & 4.132103000 & -0.263583000 & -0.525266000\end{array}$

\section{$\underline{\mathrm{G}_{1}-\text { Structure of A1-3 }}$}

$\begin{array}{lrrr}\mathrm{S} & -0.045438000 & 0.995141000 & 1.322756000 \\ \mathrm{C} & -1.302196000 & 0.570337000 & 0.070927000 \\ \mathrm{H} & -1.886125000 & 1.471046000 & -0.094192000 \\ \mathrm{H} & -0.818793000 & 0.325806000 & -0.869945000 \\ \mathrm{C} & -2.188458000 & -0.553465000 & 0.556778000 \\ \mathrm{H} & -2.730753000 & -0.266279000 & 1.448969000 \\ \mathrm{H} & -1.626363000 & -1.457498000 & 0.757213000 \\ \mathrm{C} & 1.161283000 & -0.338247000 & 1.015700000 \\ \mathrm{H} & 1.773322000 & -0.405237000 & 1.910643000 \\ \mathrm{H} & 0.636487000 & -1.283188000 & 0.908937000 \\ \mathrm{C} & 2.023699000 & -0.055004000 & -0.193826000 \\ \mathrm{H} & 1.443377000 & 0.064371000 & -1.099649000 \\ \mathrm{H} & 2.627744000 & 0.831594000 & -0.050209000\end{array}$




$\begin{array}{lrrr}\mathrm{Cl} & 3.151925000 & -1.435289000 & -0.463304000 \\ \mathrm{Cl} & -3.407584000 & -0.967457000 & -0.703968000 \\ \mathrm{O} & 0.750396000 & 2.707314000 & -1.375324000 \\ \mathrm{H} & 0.848155000 & 3.652312000 & -1.502736000 \\ \mathrm{H} & 0.637022000 & 2.591255000 & -0.424379000\end{array}$

$\underline{\mathrm{G}}_{2}-$ Structure of A2-1

$\begin{array}{lrrr}\mathrm{S} & 0.767165000 & -2.252862000 & -0.027650000 \\ \mathrm{C} & 1.735925000 & -0.820853000 & -0.603257000 \\ \mathrm{H} & 2.498375000 & -1.225574000 & -1.263374000 \\ \mathrm{H} & 1.108079000 & -0.146196000 & -1.173676000 \\ \mathrm{C} & -0.734610000 & -1.424157000 & 0.587913000 \\ \mathrm{H} & -0.475755000 & -0.551771000 & 1.178662000 \\ \mathrm{H} & -1.221917000 & -2.148073000 & 1.235462000 \\ \mathrm{C} & -1.643928000 & -1.041671000 & -0.555057000 \\ \mathrm{H} & -1.907471000 & -1.898909000 & -1.161399000 \\ \mathrm{H} & -1.212702000 & -0.259369000 & -1.165654000 \\ \mathrm{C} & 2.370047000 & -0.102814000 & 0.564391000 \\ \mathrm{H} & 1.625581000 & 0.267774000 & 1.256281000 \\ \mathrm{Cl} & -3.210332000 & -0.384552000 & 0.093825000 \\ \mathrm{H} & 3.079965000 & -0.734651000 & 1.083491000 \\ \mathrm{Cl} & 3.285266000 & 1.347446000 & -0.018329000 \\ \mathrm{O} & -0.045315000 & 1.730571000 & -0.114570000 \\ \mathrm{H} & -0.811190000 & 2.321748000 & -0.059568000 \\ \mathrm{H} & 0.725403000 & 2.302209000 & -0.144212000 \\ \mathrm{O} & -2.611964000 & 2.868357000 & 0.101361000 \\ \mathrm{H} & -3.084931000 & 3.319209000 & -0.600152000\end{array}$


$\begin{array}{llll}\mathrm{H} & -2.978330000 & 1.975745000 & 0.114850000\end{array}$

\section{$\underline{\mathrm{G}}_{2}$ - Structure of A2-2}

$\begin{array}{lrrr}\mathrm{S} & 0.076356000 & 0.306739000 & -1.679469000 \\ \mathrm{C} & -1.118218000 & -0.898053000 & -1.007562000 \\ \mathrm{H} & -1.699658000 & -1.250934000 & -1.854748000 \\ \mathrm{H} & -0.578091000 & -1.749763000 & -0.604333000 \\ \mathrm{C} & 1.336086000 & 0.291180000 & -0.358243000 \\ \mathrm{H} & 0.855399000 & 0.336508000 & 0.614309000 \\ \mathrm{H} & 1.907531000 & 1.207757000 & -0.479623000 \\ \mathrm{C} & 2.237883000 & -0.913444000 & -0.497852000 \\ \mathrm{H} & 2.767287000 & -0.902653000 & -1.442593000 \\ \mathrm{H} & 1.690344000 & -1.843815000 & -0.406086000 \\ \mathrm{C} & -2.019304000 & -0.280690000 & 0.037627000 \\ \mathrm{H} & -1.472978000 & 0.100911000 & 0.890671000 \\ \mathrm{Cl} & 3.476658000 & -0.910020000 & 0.811335000 \\ \mathrm{H} & -2.619691000 & 0.515092000 & -0.387372000 \\ \mathrm{Cl} & -3.164405000 & -1.530903000 & 0.654551000 \\ \mathrm{O} & -0.524637000 & 3.242438000 & -0.334276000 \\ \mathrm{H} & -0.518408000 & 2.456942000 & -0.902899000 \\ \mathrm{H} & -1.188603000 & 3.823413000 & -0.709750000 \\ \mathrm{H} & -0.430260000 & 1.762339000 & 2.072893000 \\ \mathrm{H} & -0.491534000 & 2.458062000 & 1.402130000 \\ \mathrm{H} & -0.161089000 & 2.204173000 & 2.878971000\end{array}$

$\underline{\mathrm{G}_{2}-\text { Structure of } \mathbf{A 2 - 3}}$

O $\quad 0.126108000 \quad 1.897286000 \quad 1.694097000$ 


$\begin{array}{lrrr}\mathrm{H} & -0.308910000 & 2.344959000 & 0.953009000 \\ \mathrm{H} & 0.213036000 & 2.564408000 & 2.376102000 \\ \mathrm{O} & -1.358705000 & 2.777099000 & -0.531700000 \\ \mathrm{H} & -1.063124000 & 2.937660000 & -1.429277000 \\ \mathrm{H} & -2.048093000 & 2.105439000 & -0.599278000 \\ \mathrm{~S} & 0.364074000 & -2.251050000 & 0.336089000 \\ \mathrm{C} & -1.033669000 & -1.128672000 & 0.664021000 \\ \mathrm{H} & -1.604655000 & -1.581094000 & 1.470015000 \\ \mathrm{H} & -0.672806000 & -0.166260000 & 1.013839000 \\ \mathrm{C} & 1.467732000 & -1.119573000 & -0.571766000 \\ \mathrm{H} & 0.889609000 & -0.514247000 & -1.264588000 \\ \mathrm{H} & 2.128688000 & -1.747756000 & -1.162559000 \\ \mathrm{C} & 2.263522000 & -0.246222000 & 0.371174000 \\ \mathrm{H} & 2.912241000 & -0.843168000 & 1.000280000 \\ \mathrm{H} & 1.630417000 & 0.387020000 & 0.979484000 \\ \mathrm{C} & -1.885301000 & -1.005863000 & -0.577560000 \\ \mathrm{H} & -1.331245000 & -0.600340000 & -1.415363000 \\ \mathrm{Cl} & 3.325053000 & 0.860375000 & -0.587374000 \\ \mathrm{H} & -2.315252000 & -1.957760000 & -0.862135000 \\ \mathrm{Cl} & -3.281995000 & 0.121674000 & -0.297999000\end{array}$

$\underline{\mathrm{G}_{2}-\text { Structure of A2-4 }}$
$\begin{array}{llll}\mathrm{S} & -0.083390000 & -1.164180000 & -0.568979000\end{array}$
$\begin{array}{llll}\text { C } & -1.607516000 & -0.714838000 & 0.324646000\end{array}$
$\begin{array}{llll}\mathrm{H} & -1.964808000 & -1.622840000 & 0.802302000\end{array}$
$\begin{array}{llll}\mathrm{H} & -1.386305000 & 0.004948000 & 1.107044000\end{array}$
$\begin{array}{llll}\text { C } & 0.670479000 & 0.484178000 & -0.775470000\end{array}$ 


$\begin{array}{lrrr}\mathrm{H} & -0.077499000 & 1.176576000 & -1.150857000 \\ \mathrm{H} & 1.439702000 & 0.356127000 & -1.529555000 \\ \mathrm{C} & 1.288721000 & 0.983379000 & 0.510091000 \\ \mathrm{H} & 2.125045000 & 0.362971000 & 0.802442000 \\ \mathrm{H} & 0.569957000 & 1.043040000 & 1.318761000 \\ \mathrm{C} & -2.652495000 & -0.179744000 & -0.628292000 \\ \mathrm{H} & -2.313643000 & 0.711862000 & -1.141486000 \\ \mathrm{Cl} & 1.924133000 & 2.652801000 & 0.265302000 \\ \mathrm{H} & -2.934295000 & -0.927423000 & -1.359156000 \\ \mathrm{Cl} & -4.141672000 & 0.275603000 & 0.279551000 \\ \mathrm{O} & 3.420843000 & -1.080828000 & -0.937445000 \\ \mathrm{H} & 4.359337000 & -1.078788000 & -1.128684000 \\ \mathrm{H} & 3.317103000 & -1.647204000 & -0.160110000 \\ \mathrm{O} & 2.323676000 & -2.359907000 & 1.335718000 \\ \mathrm{H} & 2.277675000 & -3.284608000 & 1.585520000 \\ \mathrm{H} & 1.468856000 & -2.162628000 & 0.922910000\end{array}$

$\underline{\mathrm{G}}_{2}-$ Structure of A2-5
$\begin{array}{llll}\text { S } & 0.622966000 & 1.487395000 & 0.219877000\end{array}$
$\begin{array}{llll}\text { C } & -0.423889000 & 0.130112000 & -0.395198000\end{array}$
$\mathrm{H} \quad-1.158332000 \quad 0.584172000 \quad-1.050646000$
H $\quad 0.176892000 \quad-0.569588000 \quad-0.969731000$
$\begin{array}{llll}\text { C } & 2.121261000 & 0.532678000 & 0.624711000\end{array}$
$\mathrm{H} \quad 1.848489000 \quad-0.402895000 \quad 1.105595000$
$\begin{array}{llll}\mathrm{H} & 2.682509000 & 1.119794000 & 1.345933000\end{array}$
$\begin{array}{llll}\text { C } & 2.953400000 & 0.284514000 & -0.612462000\end{array}$
$\begin{array}{llll}\mathrm{H} & 3.306923000 & 1.215275000 & -1.037972000\end{array}$ 


$\begin{array}{lrrr}\mathrm{H} & 2.403157000 & -0.265211000 & -1.366391000 \\ \mathrm{C} & -1.107876000 & -0.548604000 & 0.766146000 \\ \mathrm{H} & -0.403693000 & -0.965729000 & 1.475659000 \\ \mathrm{Cl} & 4.407065000 & -0.705065000 & -0.204009000 \\ \mathrm{H} & -1.784628000 & 0.133946000 & 1.262083000 \\ \mathrm{Cl} & -2.101458000 & -1.955412000 & 0.178222000 \\ \mathrm{O} & -2.916390000 & 1.984336000 & -0.016278000 \\ \mathrm{H} & -3.278800000 & 2.831920000 & 0.243675000 \\ \mathrm{H} & -3.683957000 & 1.429637000 & -0.220576000 \\ \mathrm{O} & -4.750779000 & -0.106282000 & -0.533224000 \\ \mathrm{H} & -4.100518000 & -0.798718000 & -0.361901000 \\ \mathrm{H} & -5.090836000 & -0.299451000 & -1.408554000\end{array}$

\section{$\underline{\mathrm{G}_{2}-\text { Structure of A2-6 }}$}

$\begin{array}{lrrr}\mathrm{O} & -2.937676000 & 1.968231000 & -0.001070000 \\ \mathrm{H} & -3.704754000 & 1.423800000 & -0.232652000 \\ \mathrm{H} & -3.234068000 & 2.874686000 & -0.088701000 \\ \mathrm{O} & -4.748190000 & -0.119118000 & -0.616601000 \\ \mathrm{H} & -4.085367000 & -0.800568000 & -0.449145000 \\ \mathrm{H} & -5.497683000 & -0.367615000 & -0.072807000 \\ \mathrm{~S} & 0.634246000 & 1.490444000 & 0.217849000 \\ \mathrm{C} & -0.413542000 & 0.137690000 & -0.405437000 \\ \mathrm{H} & -1.142918000 & 0.593467000 & -1.065250000 \\ \mathrm{H} & 0.188476000 & -0.564322000 & -0.975700000 \\ \mathrm{C} & 2.127748000 & 0.531026000 & 0.628861000 \\ \mathrm{H} & 1.849495000 & -0.404060000 & 1.107567000 \\ \mathrm{H} & 2.687239000 & 1.115811000 & 1.353331000\end{array}$




$\begin{array}{lrrr}\mathrm{C} & 2.965188000 & 0.281122000 & -0.604365000 \\ \mathrm{H} & 3.323364000 & 1.211083000 & -1.027721000 \\ \mathrm{H} & 2.417281000 & -0.266881000 & -1.361223000 \\ \mathrm{C} & -1.109313000 & -0.538470000 & 0.750400000 \\ \mathrm{H} & -0.412519000 & -0.958655000 & 1.465472000 \\ \mathrm{Cl} & 4.414072000 & -0.712418000 & -0.188660000 \\ \mathrm{H} & -1.787478000 & 0.145951000 & 1.242209000 \\ \mathrm{Cl} & -2.101281000 & -1.941166000 & 0.150052000\end{array}$

$\underline{\mathrm{G}}_{2}$ - Structure of A2-7

$\begin{array}{lrrr}\mathrm{O} & -2.311151000 & 2.839622000 & -0.715775000 \\ \mathrm{H} & -1.402247000 & 3.172380000 & -0.733464000 \\ \mathrm{H} & -2.720801000 & 3.181460000 & -1.511048000 \\ \mathrm{O} & 0.473281000 & 3.311004000 & -0.338886000 \\ \mathrm{H} & 0.740774000 & 4.054821000 & 0.204620000 \\ \mathrm{H} & 0.473397000 & 2.548538000 & 0.261149000 \\ \mathrm{~S} & 0.053162000 & 0.505239000 & 1.333509000 \\ \mathrm{C} & 1.490258000 & -0.539724000 & 0.922995000 \\ \mathrm{H} & 2.147731000 & -0.518646000 & 1.787367000 \\ \mathrm{H} & 1.161257000 & -1.565839000 & 0.784420000 \\ \mathrm{C} & 2.210887000 & -0.027699000 & -0.303876000 \\ \mathrm{H} & 2.622023000 & 0.960312000 & -0.137119000 \\ \mathrm{H} & 1.564663000 & 0.001041000 & -1.172853000 \\ \mathrm{C} & -1.148327000 & -0.128964000 & 0.114460000 \\ \mathrm{H} & -1.862635000 & 0.669627000 & -0.064271000 \\ \mathrm{H} & -0.638343000 & -0.328792000 & -0.823765000 \\ \mathrm{C} & -1.822251000 & -1.373161000 & 0.645605000\end{array}$




$\begin{array}{lrrr}\mathrm{H} & -1.110824000 & -2.157097000 & 0.877752000 \\ \mathrm{H} & -2.410044000 & -1.152232000 & 1.527941000 \\ \mathrm{Cl} & -2.945150000 & -2.041899000 & -0.595401000 \\ \mathrm{Cl} & 3.586562000 & -1.119108000 & -0.708992000\end{array}$

$\underline{\mathrm{G}}_{3-}$ - Structure of A3-1

$\begin{array}{lrrr}\mathrm{S} & -0.497912000 & -2.471789000 & -0.207346000 \\ \mathrm{C} & -1.603534000 & -1.231871000 & 0.539639000 \\ \mathrm{H} & -2.332126000 & -1.792421000 & 1.119021000 \\ \mathrm{H} & -1.048625000 & -0.594537000 & 1.219797000 \\ \mathrm{C} & -2.289252000 & -0.415624000 & -0.530781000 \\ \mathrm{H} & -2.915161000 & -1.036411000 & -1.159630000 \\ \mathrm{H} & -1.580753000 & 0.132249000 & -1.138294000 \\ \mathrm{C} & 0.943835000 & -1.439917000 & -0.629956000 \\ \mathrm{H} & 1.527056000 & -2.039316000 & -1.324087000 \\ \mathrm{H} & 0.634781000 & -0.535148000 & -1.146023000 \\ \mathrm{C} & 1.762689000 & -1.124361000 & 0.601075000 \\ \mathrm{H} & 1.260305000 & -0.425434000 & 1.258994000 \\ \mathrm{H} & 2.024688000 & -2.024898000 & 1.141809000 \\ \mathrm{Cl} & 3.334792000 & -0.348875000 & 0.127552000 \\ \mathrm{Cl} & -3.373693000 & 0.822666000 & 0.227545000 \\ \mathrm{O} & -0.094884000 & 1.930370000 & -1.485717000 \\ \mathrm{H} & -0.460437000 & 2.553073000 & -2.116560000 \\ \mathrm{H} & 0.775915000 & 2.292799000 & -1.229631000 \\ \mathrm{O} & -0.164573000 & 1.792171000 & 1.315411000 \\ \mathrm{H} & -0.484827000 & 1.877286000 & 0.403185000 \\ \mathrm{H} & -0.912959000 & 2.039967000 & 1.862101000 \\ \mathrm{O} & 2.128156000 & 2.738788000 & -0.096184000\end{array}$




$\begin{array}{llll}\mathrm{H} & 2.732912000 & 1.990667000 & -0.048310000 \\ \mathrm{H} & 1.575117000 & 2.636302000 & 0.690591000\end{array}$

$\underline{\mathrm{G}}_{3}-$ Structure of A3-2

$\begin{array}{lrrr}\mathrm{S} & -0.557853000 & -2.419050000 & 0.219288000 \\ \mathrm{C} & 0.847492000 & -1.569282000 & -0.572508000 \\ \mathrm{H} & 1.308387000 & -2.296266000 & -1.235277000 \\ \mathrm{H} & 0.493617000 & -0.736632000 & -1.174370000 \\ \mathrm{C} & 1.836931000 & -1.106953000 & 0.472111000 \\ \mathrm{H} & 2.183303000 & -1.933179000 & 1.079997000 \\ \mathrm{H} & 1.430127000 & -0.327900000 & 1.105762000 \\ \mathrm{C} & -1.596007000 & -0.984976000 & 0.651026000 \\ \mathrm{H} & -2.241341000 & -1.305264000 & 1.464329000 \\ \mathrm{H} & -0.974796000 & -0.173584000 & 1.017041000 \\ \mathrm{C} & -2.418552000 & -0.564342000 & -0.544165000 \\ \mathrm{H} & -1.791571000 & -0.277166000 & -1.378204000 \\ \mathrm{H} & -3.102758000 & -1.346315000 & -0.848945000 \\ \mathrm{Cl} & -3.426327000 & 0.884390000 & -0.135887000 \\ \mathrm{Cl} & 3.313236000 & -0.419538000 & -0.332330000 \\ \mathrm{O} & 2.225107000 & 2.710224000 & -0.069116000 \\ \mathrm{H} & 2.815697000 & 1.963407000 & -0.213382000 \\ \mathrm{H} & 1.550287000 & 2.607931000 & -0.755413000 \\ \mathrm{O} & 0.269484000 & 1.811043000 & 1.620452000 \\ \mathrm{H} & 1.070927000 & 2.232288000 & 1.252211000 \\ \mathrm{H} & 0.017157000 & 2.337428000 & 2.380864000 \\ \mathrm{O} & -0.293982000 & 1.882591000 & -1.126077000 \\ \mathrm{H} & -1.078881000 & 2.274819000 & -1.514615000 \\ \mathrm{H} & -0.456021000 & 1.905194000 & -0.169782000\end{array}$




\section{$\underline{\mathrm{G}_{3}-\text { Structure of A3-3 }}$}

\begin{tabular}{|c|c|c|c|}
\hline & 113605000 & -2.497947000 & 0.09 \\
\hline $\mathrm{C}$ & .222951000 & -1.336882000 & .529893000 \\
\hline $\mathrm{H}$ & -1.925645000 & -1.922845000 & .1167330 \\
\hline $\mathrm{H}$ & -0.850050000 & -0.537503000 & 9000 \\
\hline & 1.355665000 & -1.34 & -0.570502000 \\
\hline$H$ & 0.888 & -0.6 & 00 \\
\hline $\mathrm{H}$ & 000 & -1.9 & 00 \\
\hline $\mathrm{C}$ & 2.068 & -0.610702000 & 5000 \\
\hline 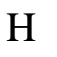 & 2.65 & -1.288396000 & 000 \\
\hline $\mathrm{H}$ & 1.403075000 & -0.028911000 & \\
\hline $\mathrm{C}$ & -1.89 & -0.8 & 00 \\
\hline $\mathrm{H}$ & -1.2 & 000 & 00 \\
\hline $\mathrm{Cl}$ & 506000 & $0.5^{\prime}$ & 000 \\
\hline $\mathrm{H}$ & -2.290853000 & 1000 & 100 \\
\hline $\mathrm{Cl}$ & 866000 & 087000 & 8000 \\
\hline $\mathrm{O}$ & -1.123495000 & 2.694650000 & 1000 \\
\hline 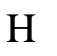 & -0.537413000 & 1000 & -0.4 \\
\hline$H$ & -1.930755000 & 5608000 & 2000 \\
\hline O & 0.682969000 & 1.853554000 & 8000 \\
\hline $\mathrm{H}$ & 0.879127000 & 2.418795000 & 589000 \\
\hline $\mathrm{H}$ & 1.541920000 & 1.658653000 & -1.340242000 \\
\hline $\mathrm{O}$ & 0.068086000 & 1.268762000 & 2.331034000 \\
\hline $\mathrm{H}$ & -0.363741000 & 1.882402000 & 1.70876800 \\
\hline & 000 & 7000 & 3.14 \\
\hline
\end{tabular}




\section{$\underline{\mathrm{G}}_{3}$ - Structure of A3-4}

$\begin{array}{lrrr}\mathrm{S} & -0.047257000 & -2.470903000 & -0.085406000 \\ \mathrm{C} & 1.149453000 & -1.273707000 & -0.763231000 \\ \mathrm{H} & 1.656394000 & -1.767103000 & -1.587625000 \\ \mathrm{H} & 0.620107000 & -0.414317000 & -1.167491000 \\ \mathrm{C} & -1.264149000 & -1.306647000 & 0.611921000 \\ \mathrm{H} & -0.757390000 & -0.488343000 & 1.115336000 \\ \mathrm{H} & -1.828055000 & -1.856315000 & 1.360351000 \\ \mathrm{C} & -2.174840000 & -0.822877000 & -0.491511000 \\ \mathrm{H} & -2.790533000 & -1.621915000 & -0.884804000 \\ \mathrm{H} & -1.624535000 & -0.359473000 & -1.301073000 \\ \mathrm{C} & 2.140010000 & -0.856470000 & 0.298933000 \\ \mathrm{H} & 1.666486000 & -0.356883000 & 1.135311000 \\ \mathrm{Cl} & -3.314705000 & 0.448412000 & 0.133253000 \\ \mathrm{H} & 2.717239000 & -1.702089000 & 0.651344000 \\ \mathrm{Cl} & 3.334010000 & 0.317625000 & -0.405433000 \\ \mathrm{O} & 1.050909000 & 2.693614000 & 0.184448000 \\ \mathrm{H} & 0.348766000 & 2.575256000 & -0.472970000 \\ \mathrm{H} & 1.813705000 & 2.224741000 & -0.169323000 \\ \mathrm{O} & -1.147336000 & 2.186431000 & -1.537050000 \\ \mathrm{H} & -1.453796000 & 2.859650000 & -2.147871000 \\ \mathrm{H} & -1.918441000 & 1.961441000 & -1.000405000 \\ \mathrm{O} & 0.349949000 & 1.083024000 & 2.316113000 \\ \mathrm{H} & 0.265013000 & 1.540742000 & 3.153200000 \\ \mathrm{H} & 0.581942000 & 1.770088000 & 1.664821000\end{array}$




\section{$\underline{\mathrm{G}}_{3-}$-Structure of A3-5}
S $\quad-1.735603000 \quad-2.155131000$
0.186713000
$\begin{array}{llll}\text { C } & -2.202412000 & -0.474722000 & 0.712575000\end{array}$
$\mathrm{H} \quad-2.971821000 \quad-0.602332000 \quad 1.469215000$
$\begin{array}{llll}\mathrm{H} & -1.354341000 & 0.025281000 & 1.166657000\end{array}$
$\begin{array}{llll}\text { C } & -2.733103000 & 0.322548000 & -0.455353000\end{array}$
H $\quad-3.642812000 \quad-0.109680000 \quad-0.853046000$
$\begin{array}{llll}\mathrm{H} & -1.994289000 & 0.423384000 & -1.239455000\end{array}$
$\begin{array}{llll}\text { C } & -0.135513000 & -1.822941000 & -0.619031000\end{array}$
$\mathrm{H} \quad 0.072415000 \quad-2.701606000 \quad-1.223793000$
$\mathrm{H} \quad-0.205142000 \quad-0.960330000 \quad-1.273401000$
$\begin{array}{llll}\text { C } & 0.951581000 & -1.617873000 & 0.408325000\end{array}$
H $\quad 0.811402000 \quad-0.693975000 \quad 0.953658000$
$\mathrm{H} \quad 1.031379000 \quad-2.461461000 \quad 1.082125000$
$\mathrm{Cl} \quad 2.557905000 \quad-1.476990000 \quad-0.429969000$
$\begin{array}{llll}\mathrm{Cl} & -3.138433000 & 2.005555000 & 0.077506000\end{array}$
$\begin{array}{llll}\text { O } & 0.172225000 & 1.446923000 & -0.196764000\end{array}$
H $\quad 0.979171000 \quad 1.979075000 \quad-0.307890000$
$\mathrm{H} \quad-0.517551000 \quad 2.094625000 \quad-0.028408000$
$\begin{array}{llll}\text { O } & 2.546419000 & 2.930341000 & -0.365511000\end{array}$
$\mathrm{H} \quad 3.209887000 \quad 2.358840000 \quad 0.055020000$
$\mathrm{H} \quad 2.919125000 \quad 3.162178000 \quad-1.217045000$
$\begin{array}{llll}\text { O } & 4.253554000 & 1.038747000 & 0.790772000\end{array}$
$\mathrm{H} \quad 4.447597000 \quad 0.996724000 \quad 1.728491000$
$\begin{array}{llll}\mathrm{H} & 3.792702000 & 0.215617000 & 0.585253000\end{array}$ 


\section{$\underline{\mathrm{G}}_{3}$ - Structure of A3-6}

$\begin{array}{lrrr}\mathrm{O} & -0.131483000 & 1.673587000 & 2.040623000 \\ \mathrm{H} & -0.778424000 & 2.185377000 & 1.537345000 \\ \mathrm{H} & -0.050912000 & 2.123918000 & 2.882573000 \\ \mathrm{O} & -1.963468000 & 2.626682000 & 0.079191000 \\ \mathrm{H} & -2.556505000 & 3.357259000 & -0.106405000 \\ \mathrm{H} & -2.487702000 & 1.822411000 & -0.050250000 \\ \mathrm{~S} & 0.600673000 & -2.428848000 & 0.364766000 \\ \mathrm{C} & -0.891281000 & -1.420109000 & 0.642661000 \\ \mathrm{H} & -1.498899000 & -1.998001000 & 1.334447000 \\ \mathrm{H} & -0.637132000 & -0.481603000 & 1.125675000 \\ \mathrm{C} & -1.635882000 & -1.197517000 & -0.651855000 \\ \mathrm{H} & -1.912762000 & -2.132166000 & -1.122788000 \\ \mathrm{H} & -1.086154000 & -0.567530000 & -1.339210000 \\ \mathrm{C} & 1.675633000 & -1.223459000 & -0.479038000 \\ \mathrm{H} & 2.485477000 & -1.806865000 & -0.909018000 \\ \mathrm{H} & 1.137599000 & -0.742398000 & -1.287976000 \\ \mathrm{C} & 2.212363000 & -0.201825000 & 0.495597000 \\ \mathrm{H} & 1.428522000 & 0.366196000 & 0.979673000 \\ \mathrm{H} & 2.849535000 & -0.661910000 & 1.240935000 \\ \mathrm{Cl} & 3.236788000 & 1.010471000 & -0.385963000 \\ \mathrm{Cl} & -3.204123000 & -0.326214000 & -0.324114000 \\ \mathrm{O} & 0.119941000 & 1.476578000 & -1.578068000 \\ \mathrm{H} & 1.019919000 & 1.724110000 & -1.344849000 \\ \mathrm{H} & -0.443563000 & 2.083096000 & -1.083268000 \\ & & \end{array}$




\section{$\underline{\mathrm{G}}_{3}-$ Structure of A3-7}

$\begin{array}{lrrr}\mathrm{S} & 0.418841000 & -1.256958000 & 0.917390000 \\ \mathrm{C} & 1.851149000 & -1.005092000 & -0.180248000 \\ \mathrm{H} & 2.265186000 & -1.998068000 & -0.334166000 \\ \mathrm{H} & 1.536429000 & -0.626941000 & -1.147705000 \\ \mathrm{C} & 2.884901000 & -0.101827000 & 0.450042000 \\ \mathrm{H} & 3.253761000 & -0.517651000 & 1.379771000 \\ \mathrm{H} & 2.497876000 & 0.893950000 & 0.631403000 \\ \mathrm{C} & -0.232432000 & 0.440819000 & 1.083722000 \\ \mathrm{H} & -1.165268000 & 0.318310000 & 1.626962000 \\ \mathrm{H} & 0.440776000 & 1.040972000 & 1.688360000 \\ \mathrm{C} & -0.505789000 & 1.101647000 & -0.246540000 \\ \mathrm{H} & 0.399749000 & 1.260265000 & -0.820813000 \\ \mathrm{H} & -1.221920000 & 0.546525000 & -0.839331000 \\ \mathrm{Cl} & -1.208365000 & 2.739289000 & 0.033174000 \\ \mathrm{Cl} & 4.293780000 & 0.090249000 & -0.656735000 \\ \mathrm{O} & -3.518956000 & -0.269545000 & 0.933230000 \\ \mathrm{H} & -4.231724000 & 0.166796000 & 1.401913000 \\ \mathrm{H} & -3.617054000 & -0.009471000 & 0.004236000 \\ \mathrm{O} & -2.412109000 & -2.568397000 & -0.380093000 \\ \mathrm{H} & -2.902316000 & -2.130175000 & 0.328748000 \\ \mathrm{H} & -1.490840000 & -2.402632000 & -0.140070000 \\ \mathrm{O} & -3.118439000 & -0.346453000 & -1.836665000 \\ \mathrm{H} & -3.628436000 & -0.412269000 & -2.644880000 \\ \mathrm{H} & -2.880666000 & -1.258563000 & -1.585760000\end{array}$




\section{$\underline{\mathrm{G}}_{3}-$ Structure of A3-8}

$\begin{array}{lrrr}\mathrm{S} & -1.025463000 & -1.198035000 & 0.836826000 \\ \mathrm{C} & -0.174851000 & 0.065817000 & -0.160815000 \\ \mathrm{H} & 0.734344000 & -0.395336000 & -0.532556000 \\ \mathrm{H} & -0.791528000 & 0.348600000 & -1.009338000 \\ \mathrm{C} & 0.167676000 & 1.262588000 & 0.694697000 \\ \mathrm{H} & 0.871553000 & 0.992981000 & 1.471090000 \\ \mathrm{H} & -0.709435000 & 1.720636000 & 1.135270000 \\ \mathrm{C} & -2.713281000 & -0.512839000 & 0.810393000 \\ \mathrm{H} & -3.237192000 & -0.940105000 & 1.660517000 \\ \mathrm{H} & -2.681859000 & 0.563819000 & 0.952389000 \\ \mathrm{C} & -3.420105000 & -0.870303000 & -0.477206000 \\ \mathrm{H} & -2.896000000 & -0.489964000 & -1.345541000 \\ \mathrm{H} & -3.537391000 & -1.942367000 & -0.573429000 \\ \mathrm{Cl} & -5.071499000 & -0.143751000 & -0.505874000 \\ \mathrm{Cl} & 0.956275000 & 2.550491000 & -0.309242000 \\ \mathrm{O} & 4.719447000 & -1.706464000 & -1.480495000 \\ \mathrm{H} & 4.964279000 & -1.527447000 & -2.388979000 \\ \mathrm{H} & 4.001036000 & -1.087262000 & -1.275399000 \\ \mathrm{O} & 5.674811000 & -0.568727000 & 0.898071000 \\ \mathrm{H} & 5.644290000 & -1.116183000 & 0.097589000 \\ \mathrm{H} & 5.901227000 & -1.168347000 & 1.609961000 \\ \mathrm{O} & 3.116474000 & 0.092630000 & -0.026221000 \\ \mathrm{H} & 2.939445000 & 1.024259000 & -0.184545000 \\ \mathrm{H} & 3.920959000 & 0.059617000 & 0.517440000\end{array}$




\section{$\underline{\mathrm{G}}_{3}-$ Structure of A3-9}

$\begin{array}{lrrr}\mathrm{S} & 0.573079000 & -1.087439000 & 1.605676000 \\ \mathrm{C} & -0.926619000 & -0.535631000 & 0.725525000 \\ \mathrm{H} & -1.564022000 & -0.072518000 & 1.474422000 \\ \mathrm{H} & -0.658371000 & 0.229649000 & 0.003130000 \\ \mathrm{C} & -1.623143000 & -1.713790000 & 0.088211000 \\ \mathrm{H} & -1.939701000 & -2.439091000 & 0.827115000 \\ \mathrm{H} & -1.007029000 & -2.201673000 & -0.657017000 \\ \mathrm{C} & 1.732518000 & -1.234379000 & 0.203483000 \\ \mathrm{H} & 2.525051000 & -1.897959000 & 0.538280000 \\ \mathrm{H} & 1.230001000 & -1.720835000 & -0.627732000 \\ \mathrm{C} & 2.295734000 & 0.106484000 & -0.211232000 \\ \mathrm{H} & 1.530062000 & 0.804913000 & -0.527774000 \\ \mathrm{H} & 2.876726000 & 0.547410000 & 0.590403000 \\ \mathrm{Cl} & 3.416927000 & -0.128614000 & -1.605395000 \\ \mathrm{Cl} & -3.123097000 & -1.171167000 & -0.772703000 \\ \mathrm{O} & -2.782129000 & 2.190560000 & -1.191837000 \\ \mathrm{H} & -3.061882000 & 2.466453000 & -2.066529000 \\ \mathrm{H} & -2.952473000 & 1.242952000 & -1.163288000 \\ \mathrm{O} & 0.003558000 & 2.454648000 & -0.452081000 \\ \mathrm{H} & 0.020245000 & 2.584962000 & 0.504161000 \\ \mathrm{H} & -0.928667000 & 2.520334000 & -0.697419000 \\ \mathrm{O} & 0.226204000 & 2.097401000 & 2.420407000 \\ \mathrm{H} & 0.593961000 & 1.203513000 & 2.384734000 \\ \mathrm{H} & 0.719708000 & 2.550220000 & 3.106509000\end{array}$


2.2. Structures of complexes obtained using B3LYP/6-311++G(2d,2p)

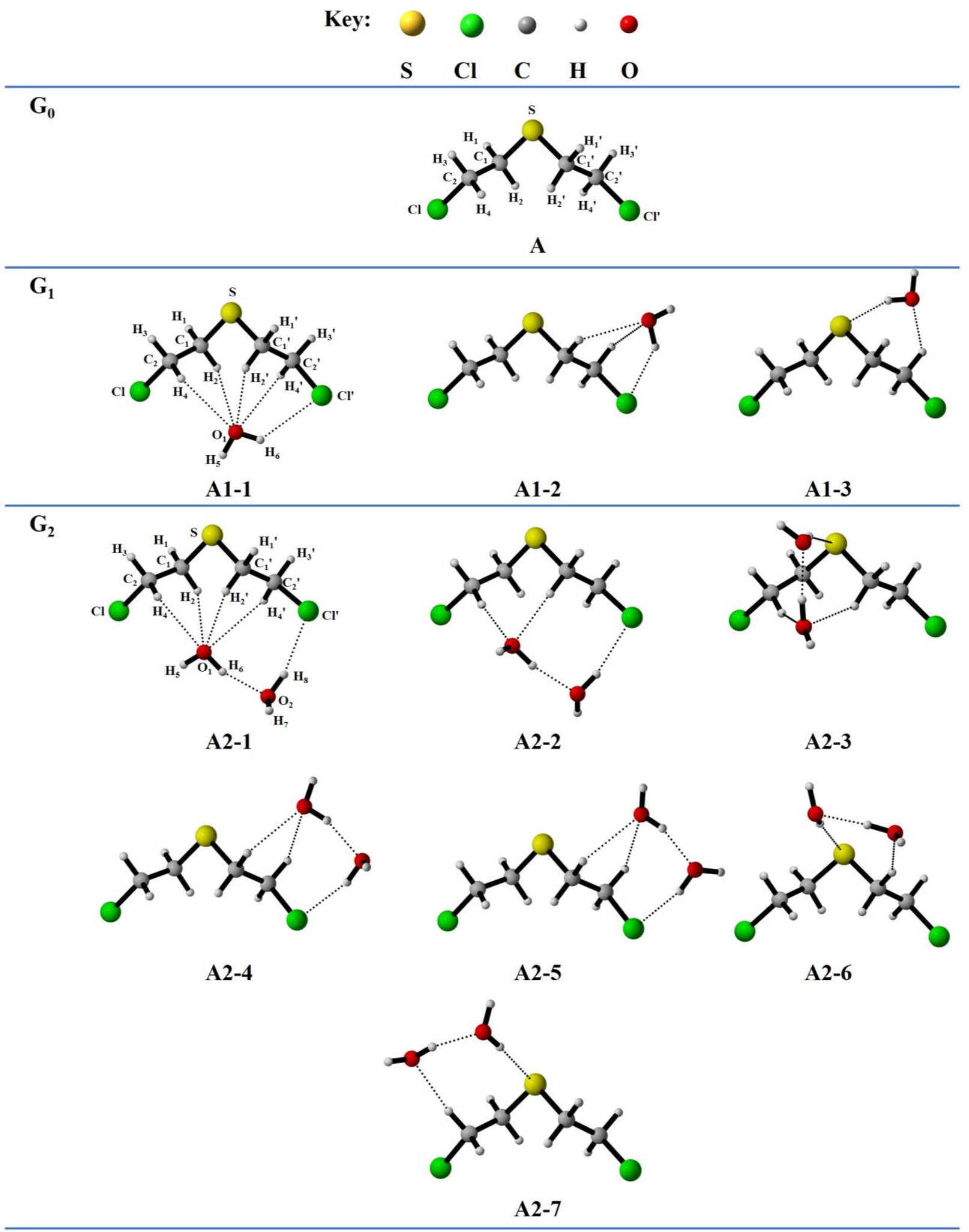

Figure S2: Complexes of SM. $n \mathrm{H}_{2} \mathrm{O}$ (for $n=1-3$ ) obtained using B3LYP/6-311++G(2d,2p), arranged in order of decreasing relative stability within each generation 


$$
\mathbf{G}_{3}
$$
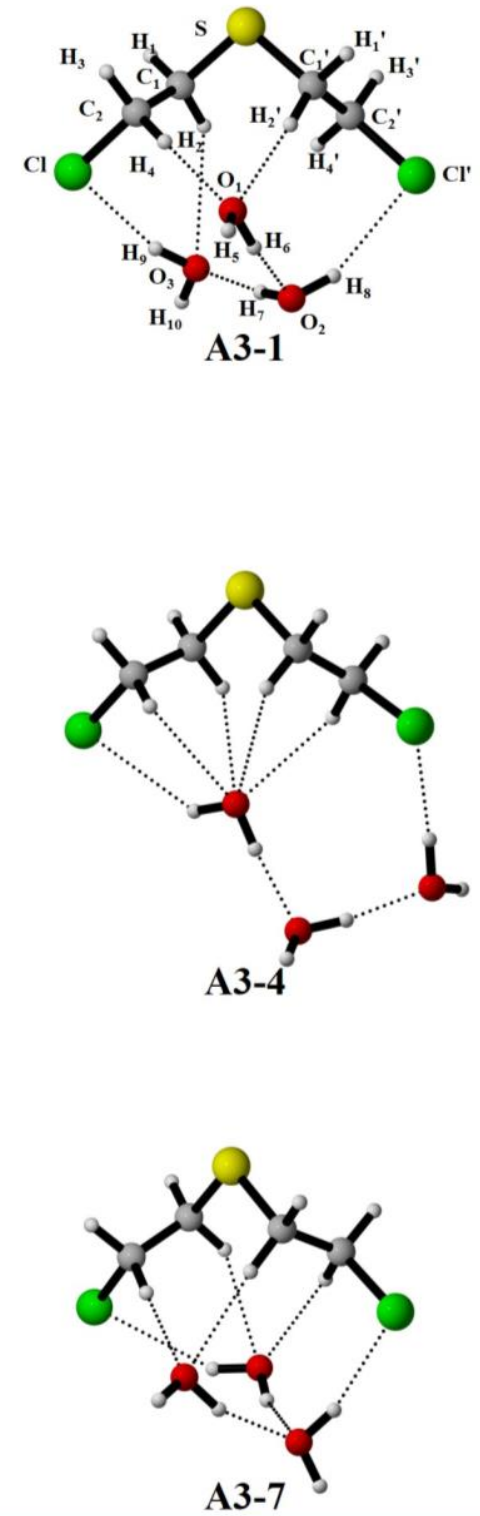

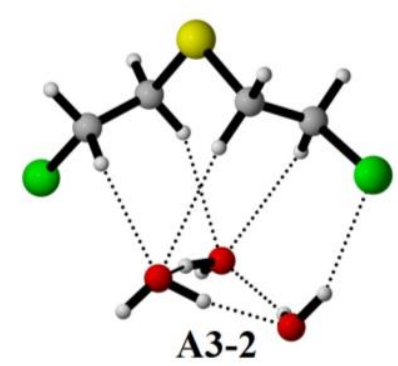

A3-2

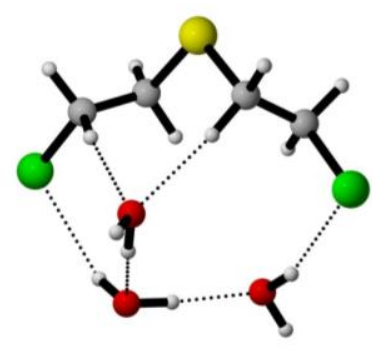

A3-5
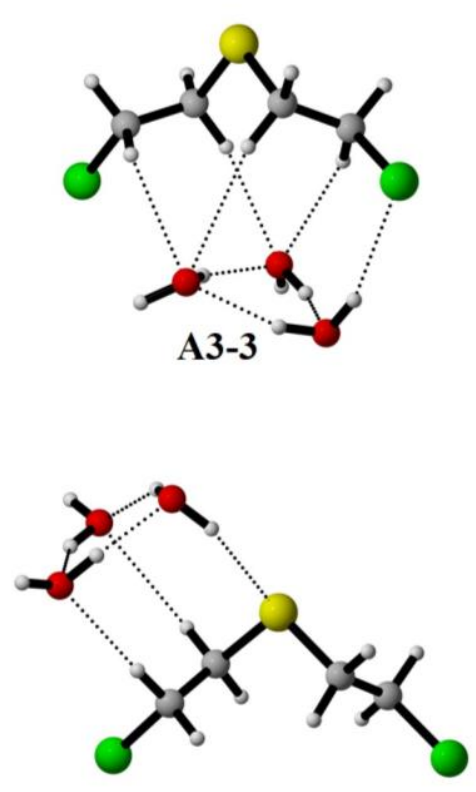

A3-6

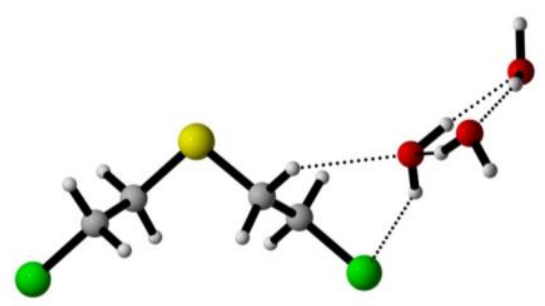

A3-8

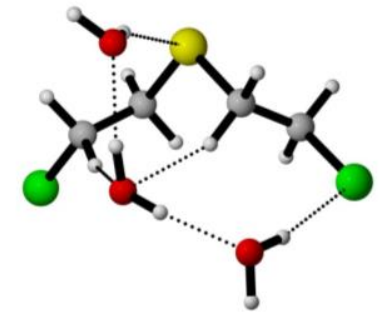

A3-9

Figure S2 (continued): Complexes of SM. $n \mathrm{H}_{2} \mathrm{O}$ (for $n=1-3$ ) obtained using B3LYP/6$311++\mathrm{G}(2 \mathrm{~d}, 2 \mathrm{p})$, arranged in order of decreasing relative stability within each generation 


\subsection{Structures of complexes obtained using B3LYP-D3/6-311++G(2d,2p)}

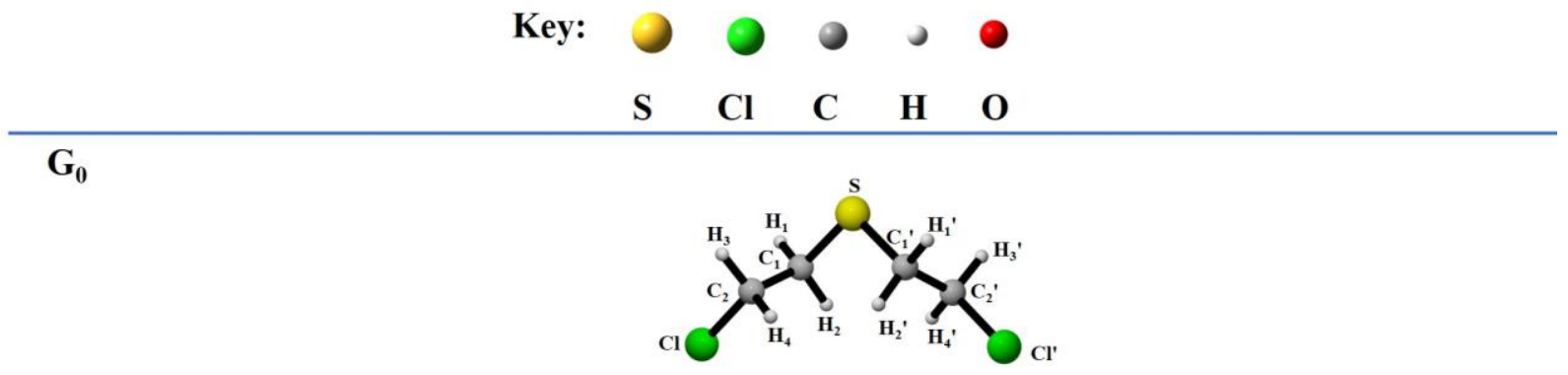

A

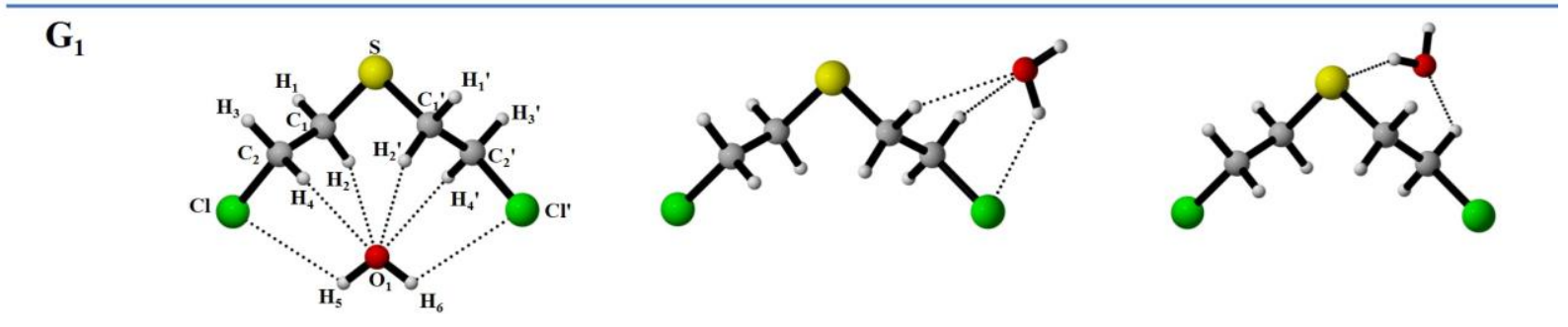

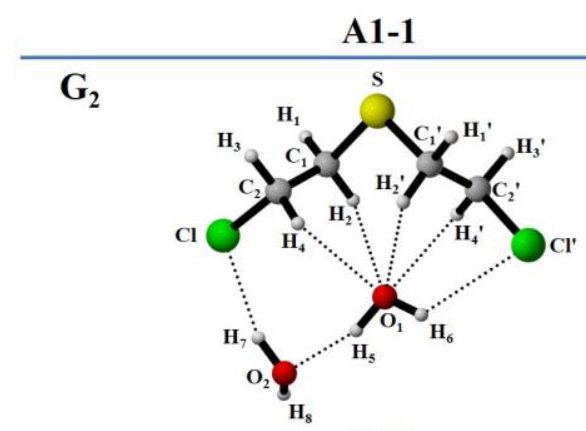

A2-1

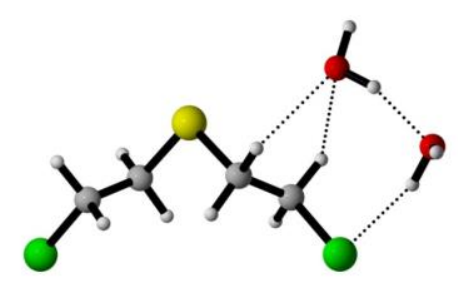

A2-4
A1-2

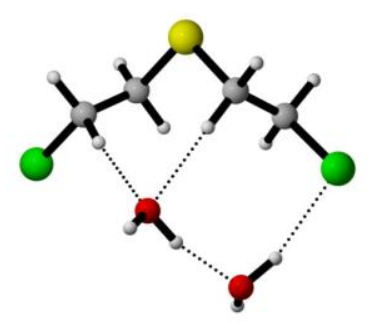

A2-2

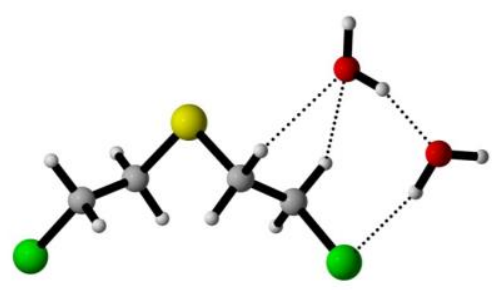

A2-5

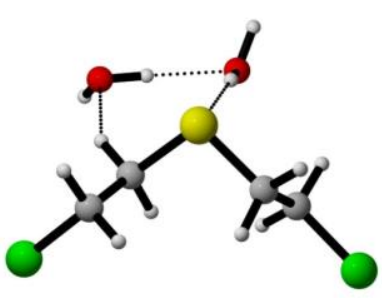

A2-7
A1-3

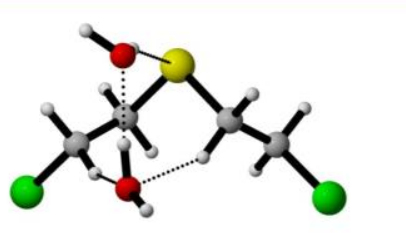

A2-3

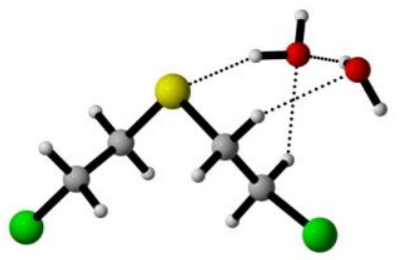

A2-6

Figure S3: Complexes of SM. $n \mathrm{H}_{2} \mathrm{O}$ (for $n=1-3$ ) obtained using B3LYP-D3/6-311++G(2d,2p), arranged in order of decreasing relative stability within each generation. 
$\mathbf{G}_{3}$

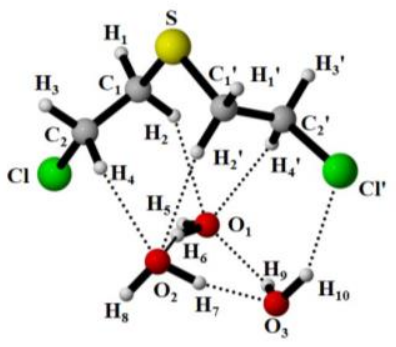

A3-1

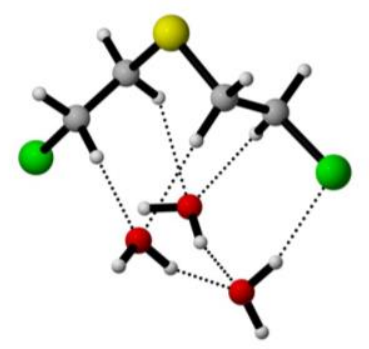

A3-4

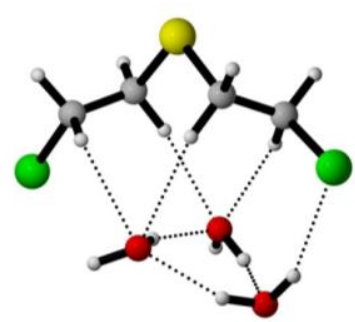

A3-2

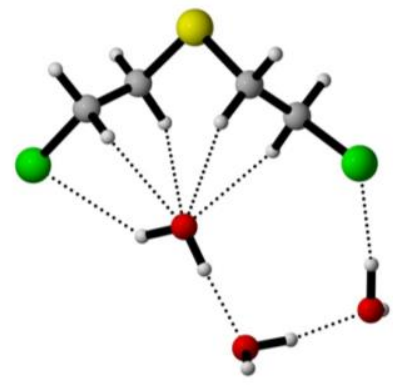

A3-5

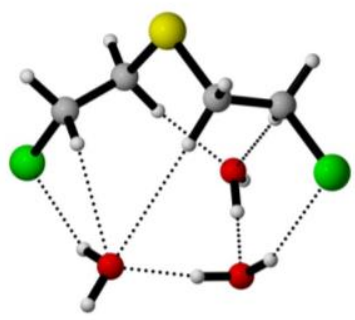

A3-3

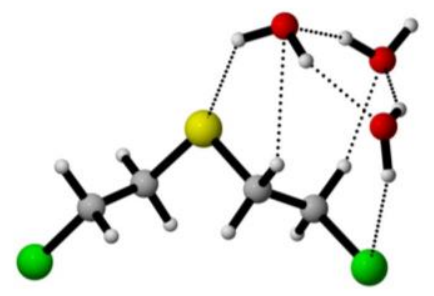

A3-6

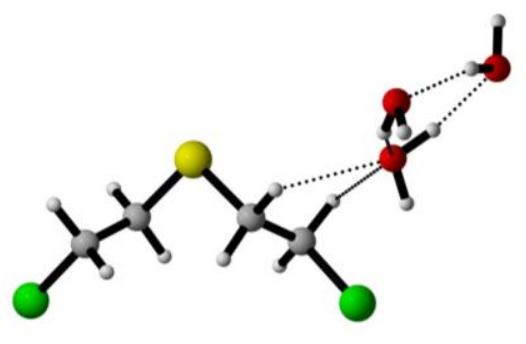

A3-7

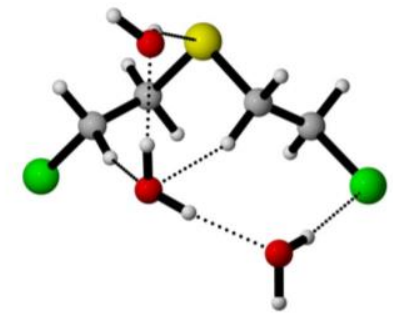

A3-8

Figure S3 (continued): Complexes of SM. $n \mathrm{H}_{2} \mathrm{O}$ (for $n=1-3$ ) obtained using B3LYP-D3/6$311++\mathrm{G}(2 \mathrm{~d}, 2 \mathrm{p})$, arranged in order of decreasing relative stability within each generation 
2.4. Structures of complexes obtained using MP2/6-311++G(2d,2p)

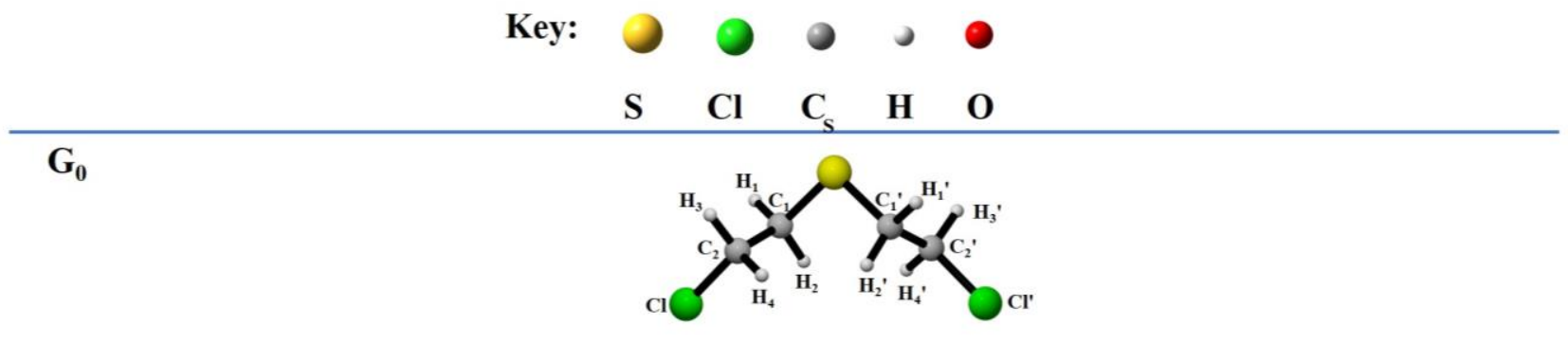

A

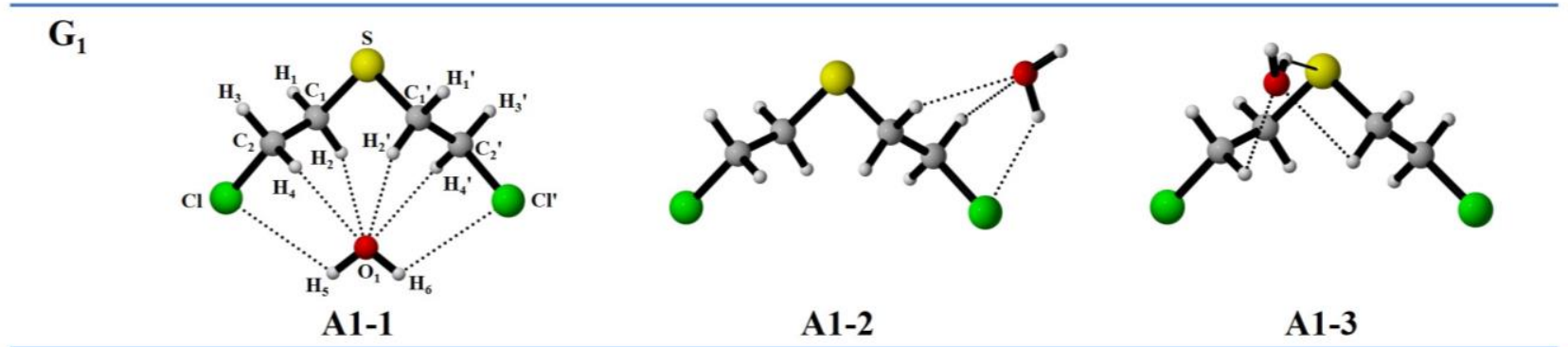

$\mathbf{G}_{2}$

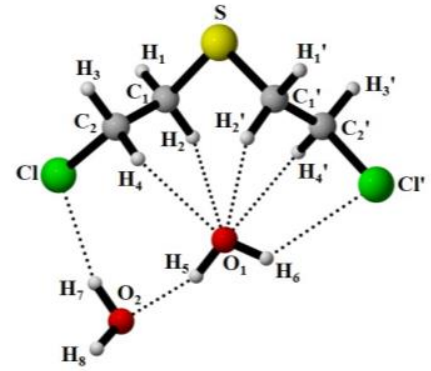

A2-1

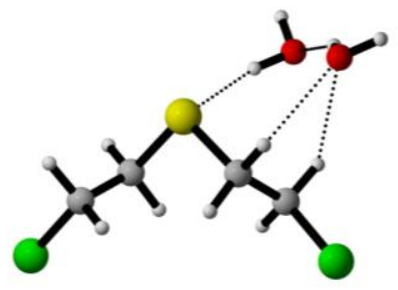

A2-4

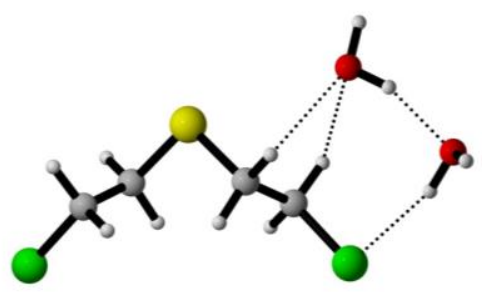

A2-5

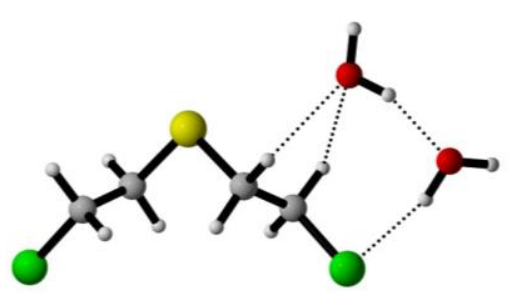

A2-6

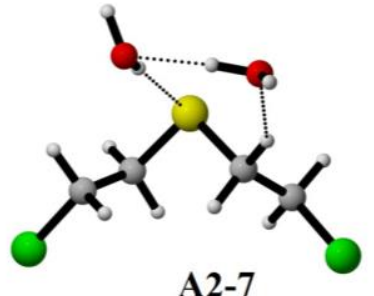

Figure S4: Complexes obtained using MP2/6-311++G(2d,2p) 


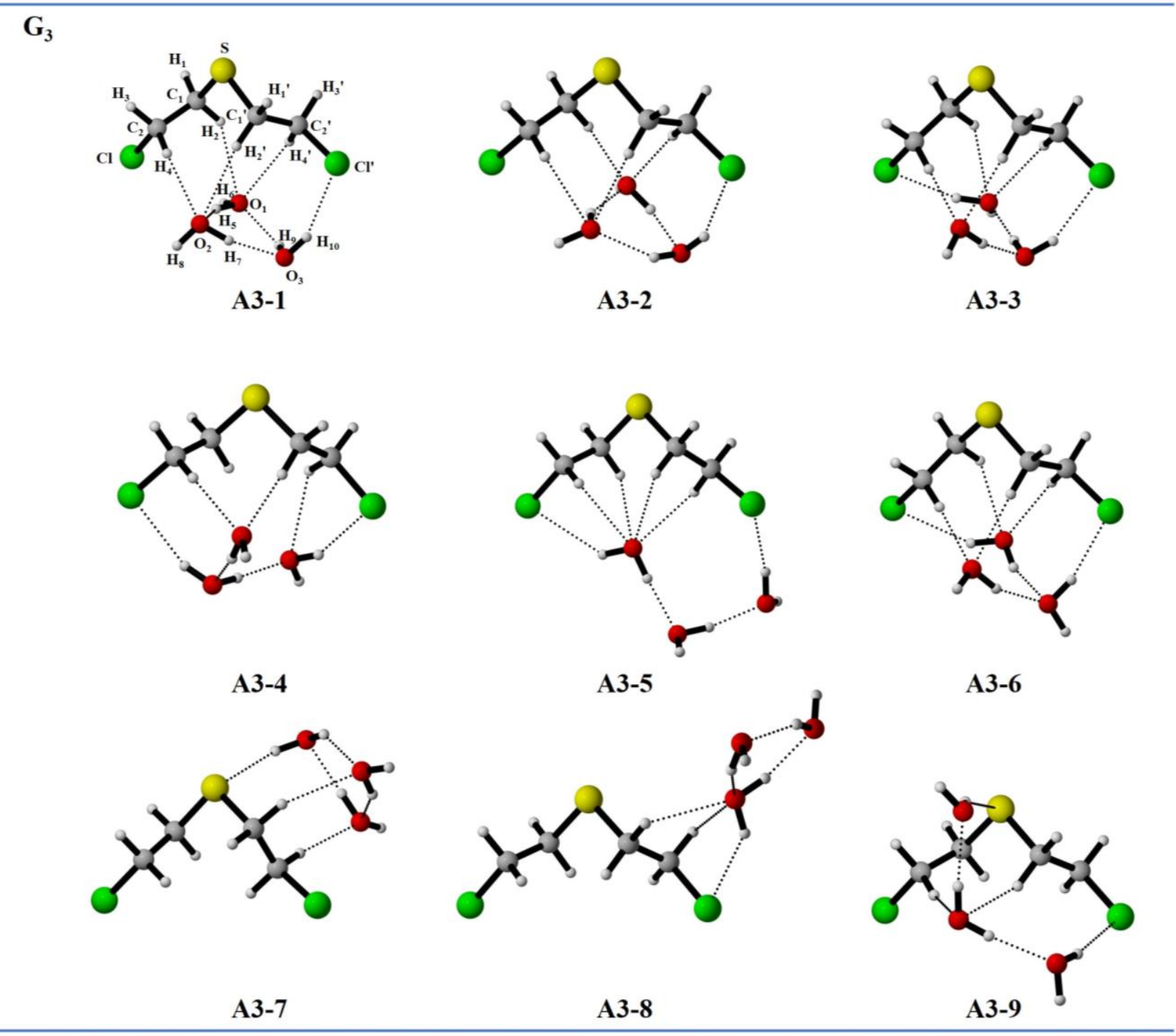

Figure S4 (continued): Complexes obtained using MP2/6-311++G(2d,2p) 

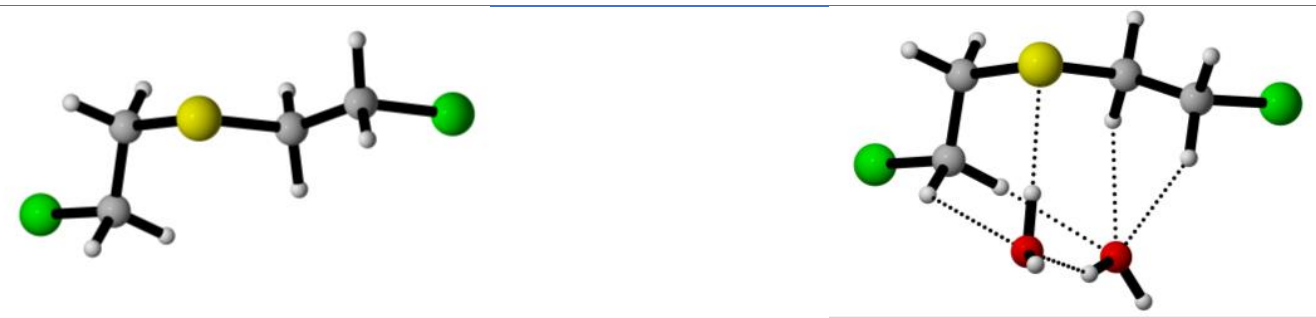

Figure S5: Structure of non-hydrated C conformer and a dihydrated complex obtained at M06$2 \mathrm{X} / 6-311++\mathrm{G}(2 \mathrm{~d}, 2 \mathrm{p})$ with the $\mathrm{C}$ structure 


\section{Effect of microhydration on the energy of SM}

The BSSE corrected binding electronic energy, $\Delta \mathrm{E}_{\mathrm{BE}}^{\mathrm{ZPE}+\mathrm{BSSE}}$, the binding electronic energy, $\Delta \mathrm{E}_{\mathrm{BE}}^{\mathrm{ZPE}}$, the enthalpy, $\Delta \mathrm{H}_{\mathrm{BE}}$, the entropy, $\Delta \mathrm{S}_{\mathrm{BE}}$ and the Gibbs free binding energy, $\Delta \mathrm{G}_{\mathrm{BE}}$ of the complexes optimised at B3LYP, B3LYP-D3 and MP2 using the 6-311++G(2d,2p) basis set are listed in Tables S2 to S4.

Table S2: The $\Delta \mathrm{E}_{\mathrm{BE}}^{\mathrm{ZPE}+\mathrm{BSSE}}, \Delta \mathrm{E}_{\mathrm{BE}}^{\mathrm{ZPE}}, \Delta \mathrm{H}_{\mathrm{BE}}, \Delta \mathrm{S}_{\mathrm{BE}}$ and $\Delta \mathrm{G}_{\mathrm{BE}}$ of all the complexes obtained using B3LYP/6-311++G(2d,2p) method.

\begin{tabular}{|c|c|c|c|c|c|c|}
\hline Generation & Cluster & $\begin{array}{c}\Delta \mathbf{E}_{\mathrm{BE}}^{\mathrm{ZPE}+\mathrm{BSSE}} / \\
(\mathbf{k c a l} / \mathbf{m o l})\end{array}$ & $\begin{array}{c}\Delta \mathbf{E}_{\mathbf{B E}}^{\mathrm{ZPE}} / \\
\text { (kcal/mol) }\end{array}$ & $\begin{array}{c}\Delta \mathrm{H}_{\mathrm{BE}} / \\
(\mathrm{kcal} / \mathrm{mol})\end{array}$ & $\begin{array}{c}\Delta \mathrm{S}_{\mathrm{BE}} / \\
(\mathrm{cal} / \mathrm{mol} / \mathrm{K})\end{array}$ & $\begin{array}{c}\Delta \mathrm{GBE}_{\mathrm{BE}} / \\
(\mathrm{kcal} / \mathrm{mol})\end{array}$ \\
\hline $\mathbf{G}_{0}$ & $\overline{\mathbf{A}}$ & 0 & 0 & 0 & 0 & 0 \\
\hline \multirow[t]{3}{*}{$\mathbf{G}_{1}$} & A1-1 & -2.34 & -2.85 & 1.96 & -24.89 & 5.15 \\
\hline & A1-2 & -1.60 & -1.95 & 3.04 & -20.58 & 4.94 \\
\hline & A1-3 & -1.30 & -1.67 & 3.17 & -21.93 & 5.48 \\
\hline \multirow[t]{7}{*}{$\mathbf{G}_{2}$} & A2-1 & -7.15 & -8.26 & -4.30 & -54.76 & 12.30 \\
\hline & A2-2 & -6.68 & -7.73 & -3.40 & -53.65 & 12.60 \\
\hline & A2-3 & -6.04 & -7.09 & -2.79 & -54.26 & 13.39 \\
\hline & A2-4 & -5.87 & -6.79 & -2.35 & -50.27 & 12.64 \\
\hline & A2-5 & -5.85 & -6.77 & -2.30 & -49.73 & 12.52 \\
\hline & A2-6 & -5.55 & -6.47 & -2.15 & -51.69 & 13.26 \\
\hline & A2-7 & -5.46 & -6.42 & -2.16 & -52.87 & 13.60 \\
\hline \multirow[t]{8}{*}{$\mathbf{G}_{3}$} & A3-1 & -12.25 & -14.14 & -10.59 & -88.20 & 15.71 \\
\hline & A3-2 & -12.19 & -14.04 & -10.71 & -88.94 & 15.81 \\
\hline & A3-3 & -12.03 & -13.91 & -10.63 & -89.26 & 15.98 \\
\hline & A3-4 & -11.92 & -13.55 & -9.86 & -82.72 & 14.80 \\
\hline & A3-5 & -11.91 & -13.75 & -10.20 & -88.34 & 16.14 \\
\hline & A3-6 & -10.60 & -12.23 & -8.72 & -84.22 & 16.39 \\
\hline & A3-7 & -10.60 & -12.40 & -8.62 & -87.39 & 17.43 \\
\hline & A3-8 & -10.51 & -11.93 & -8.36 & -80.19 & 15.55 \\
\hline
\end{tabular}



A3-9
$-9.08$
$-10.74$
$-6.77$
$-82.87$
17.94

Table S3: The $\Delta \mathrm{E}_{\mathrm{BE}}^{\mathrm{ZPE}+\mathrm{BSSE}}, \Delta \mathrm{E}_{\mathrm{BE}}^{\mathrm{ZPE}}, \Delta \mathrm{H}_{\mathrm{BE}}, \Delta \mathrm{S}_{\mathrm{BE}}$ and $\Delta \mathrm{G}_{\mathrm{BE}}$ of all the complexes obtained using B3LYP-D3/6-311++G(2d,2p) method

\begin{tabular}{|c|c|c|c|c|c|c|}
\hline Generation & Cluster & $\begin{array}{c}\Delta \mathbf{E}_{\mathbf{B E}}^{\mathrm{ZPE}+\mathrm{BSSE}} / \\
(\mathrm{kcal} / \mathbf{m o l})\end{array}$ & $\begin{array}{c}\Delta \mathbf{E}_{\mathrm{BE}}^{\mathrm{ZPE}} / \\
(\mathrm{kcal} / \mathbf{m o l})\end{array}$ & $\begin{array}{c}\Delta \mathrm{H}_{\mathrm{BE}} / \\
(\mathrm{kcal} / \mathrm{mol})\end{array}$ & $\begin{array}{c}\Delta \mathrm{S}_{\mathrm{BE}} / \\
(\mathrm{cal} / \mathrm{mol} / \mathrm{K})\end{array}$ & $\begin{array}{c}\Delta \mathrm{GBE}_{\mathrm{BE}} \\
(\mathrm{kcal} / \mathrm{mol})\end{array}$ \\
\hline $\mathbf{G}_{\mathbf{0}}$ & $\mathbf{A}$ & 0 & 0 & 0 & 0 & 0 \\
\hline \multirow[t]{3}{*}{$\mathbf{G}_{1}$} & A1-1 & -5.83 & -6.44 & -6.68 & -31.10 & 2.60 \\
\hline & A1-2 & -3.56 & -3.95 & -3.91 & -24.09 & 3.28 \\
\hline & A1-3 & -3.46 & -3.87 & -3.90 & -21.50 & 2.51 \\
\hline \multirow[t]{7}{*}{$\mathbf{G}_{2}$} & A2-1 & -12.21 & -13.40 & -14.24 & -58.99 & 3.35 \\
\hline & A2-2 & -10.54 & -11.69 & -12.19 & -53.69 & 3.82 \\
\hline & A2-3 & -10.41 & -11.55 & -12.16 & -56.15 & 4.58 \\
\hline & A2-4 & -9.40 & -10.39 & -10.89 & -52.99 & 4.90 \\
\hline & A2-5 & -9.37 & -10.36 & -10.85 & -52.62 & 4.84 \\
\hline & A2-6 & -9.14 & -10.28 & -11.00 & -56.37 & 5.81 \\
\hline & A2-7 & -9.01 & -10.02 & -10.56 & -52.77 & 5.17 \\
\hline \multirow[t]{8}{*}{$\mathbf{G}_{3}$} & A3-1 & -20.23 & -22.27 & -23.98 & -93.86 & 4.01 \\
\hline & A3-1 & -20.23 & -22.27 & -23.98 & -93.91 & 4.02 \\
\hline & A3-2 & -19.93 & -21.99 & -23.78 & -94.45 & 4.38 \\
\hline & A3-3 & -18.87 & -20.83 & -22.24 & -90.04 & 4.61 \\
\hline & A3-4 & -18.54 & -20.47 & -21.65 & -89.79 & 5.12 \\
\hline & A3-5 & -17.91 & -19.66 & -21.04 & -87.62 & 5.09 \\
\hline & A3-6 & -16.42 & -18.26 & -19.72 & -88.75 & 6.74 \\
\hline & A3-7 & -15.74 & -17.51 & -18.52 & -84.83 & 6.78 \\
\hline
\end{tabular}



A3-8
$-14.99$
$-16.45$
$-17.72$
$-80.48$
6.27

Table S4: The $\Delta \mathrm{E}_{\mathrm{BE}}^{\mathrm{ZPE}+\mathrm{BSSE}}, \Delta \mathrm{E}_{\mathrm{BE}}^{\mathrm{ZPE}}, \Delta \mathrm{H}_{\mathrm{BE}}, \Delta \mathrm{S}_{\mathrm{BE}}$ and $\Delta \mathrm{G}_{\mathrm{BE}}$ of all the complexes obtained using MP2/6-311++G(2d,2p) method

\begin{tabular}{|c|c|c|c|c|c|c|}
\hline Generation & Cluster & $\begin{array}{c}\Delta \mathbf{E}_{\mathrm{BE}}^{\mathrm{ZPE}+\mathrm{BSSE}} / \\
(\mathrm{kcal} / \mathrm{mol})\end{array}$ & $\begin{array}{c}\Delta \mathbf{E}_{\mathrm{BE}}^{\mathrm{ZPE}} / \\
(\mathrm{kcal} / \mathbf{m o l})\end{array}$ & $\begin{array}{c}\Delta \mathrm{HBE} / \\
(\mathrm{kcal} / \mathrm{mol})\end{array}$ & $\begin{array}{c}\Delta \mathrm{S}_{\mathrm{BE}} / \\
(\mathrm{cal} / \mathrm{mol} / \mathrm{K})\end{array}$ & $\begin{array}{c}\Delta \mathrm{GBE} / \\
(\mathrm{kcal} / \mathrm{mol})\end{array}$ \\
\hline $\mathbf{G}_{0}$ & $\mathbf{A}$ & 0 & 0 & 0 & 0 & 0 \\
\hline \multirow[t]{3}{*}{$\mathbf{G}_{1}$} & A1-1 & -4.45 & -6.27 & -6.42 & -29.46 & 2.36 \\
\hline & A1-2 & -1.81 & -2.99 & -2.93 & -23.69 & 4.13 \\
\hline & A1-3 & -1.71 & -3.09 & -3.08 & -23.35 & 3.88 \\
\hline \multirow[t]{7}{*}{$\mathbf{G}_{2}$} & A2-1 & -8.84 & -12.30 & -13.08 & -58.41 & 4.34 \\
\hline & A2-2 & -7.50 & -10.75 & -11.27 & -54.60 & 5.01 \\
\hline & A2-3 & -7.42 & -10.71 & -11.16 & -52.93 & 4.62 \\
\hline & A2-4 & -6.94 & -9.91 & -10.32 & -51.79 & 5.12 \\
\hline & A2-5 & -6.85 & -9.61 & -10.06 & -51.31 & 5.24 \\
\hline & A2-6 & -6.83 & -9.58 & -10.02 & -51.15 & 5.23 \\
\hline & A2-7 & -6.43 & -9.26 & -9.75 & -51.35 & 5.57 \\
\hline \multirow[t]{8}{*}{$\mathbf{G}_{3}$} & A3-1 & -14.42 & -19.99 & -21.54 & -90.51 & 5.44 \\
\hline & A3-2 & -14.25 & -19.86 & -21.49 & -91.66 & 5.84 \\
\hline & A3-3 & -13.69 & -19.51 & -20.97 & -91.15 & 6.21 \\
\hline & A3-4 & -13.40 & -18.98 & -19.57 & -86.62 & 6.25 \\
\hline & A3-5 & -13.32 & -18.30 & -20.38 & -89.80 & 6.39 \\
\hline & A3-6 & -13.10 & -18.65 & -19.85 & -90.18 & 7.04 \\
\hline & A3-7 & -11.94 & -16.91 & -16.29 & -79.83 & 7.51 \\
\hline & A3-8 & -11.18 & -15.08 & -18.38 & -87.93 & 7.84 \\
\hline
\end{tabular}


$\begin{array}{llllll}\text { A3-9 } & -10.92 & -15.82 & -16.78 & -84.25 & 8.34\end{array}$

(15.9.




\section{Effect of microhydration on the structural parameters of SM}

Tables S5 to S7 show the effect of adding 1 to 3 discrete water molecules to $\mathbf{A}$ at different levels of theory. In each case, the changes to the geometry of $\mathbf{A}$ after microhydration is negligible. The bond lengths and angles of the hydrogen bonding formed between the $1-3$ water molecules in the most stable complexes of each generation, A1-1, A2-1 and A3-1 at the three different levels of theory are shown in Tables S8 to S10. The values of the hydrogen bond lengths and angles obtained indicate weak interactions between the CWA and water in all the complexes considered.

Table S5: Structural parameters of the most stable SM. $n \mathrm{H}_{2} \mathrm{O}$ clusters optimised using B3LYP/6$311++\mathrm{G}(2 \mathrm{~d}, 2 \mathrm{p})$

\begin{tabular}{|c|c|c|c|c|}
\hline Bonds & Isolated A & A1-1 & A2-1 & A3-1 \\
\hline $\mathrm{C}_{2}-\mathrm{Cl} / \AA$ & 1.83 & 1.84 & 1.84 & 1.84 \\
\hline $\mathrm{C}_{2}{ }^{\prime}-\mathrm{Cl}^{\prime} / \mathrm{A}$ & 1.83 & 1.83 & 1.83 & 1.85 \\
\hline $\mathrm{C}_{1}-\mathrm{C}_{2} / \AA$ & 1.52 & 1.51 & 1.51 & 1.51 \\
\hline $\mathbf{C}_{1}{ }^{\prime}-\mathbf{C}_{2}{ }^{\prime} / \mathbf{A}$ & 1.52 & 1.51 & 1.51 & 1.51 \\
\hline $\mathbf{S}-\mathbf{C}_{1} / \mathbf{A}$ & 1.84 & 1.84 & 1.84 & 1.84 \\
\hline $\mathbf{S}-\mathbf{C}_{1}{ }^{\prime} / \mathbf{A}$ & 1.84 & 1.84 & 1.84 & 1.84 \\
\hline $\mathbf{C}_{1}-\mathbf{H}_{1} / \AA ̊ ̊$ & 1.09 & 1.09 & 1.09 & 1.09 \\
\hline $\mathbf{C}_{1}{ }^{\prime}-\mathbf{H}_{1}{ }^{\prime} / \mathbf{A}$ & 1.09 & 1.09 & 1.09 & 1.09 \\
\hline $\mathbf{C}_{1}-\mathbf{H}_{2} / \AA$ & 1.09 & 1.09 & 1.09 & 1.09 \\
\hline $\mathbf{C}^{\prime}{ }^{\prime}-\mathbf{H}_{2}^{\prime} / \AA$ & 1.09 & 1.09 & 1.09 & 1.09 \\
\hline $\mathrm{C}_{2}-\mathrm{H}_{3} / \mathbf{A}$ & 1.08 & 1.08 & 1.08 & 1.09 \\
\hline $\mathbf{C}_{2}^{\prime}-\mathbf{H}_{3}^{\prime} / \mathbf{A}$ & 1.08 & 1.08 & 1.08 & 1.08 \\
\hline $\mathrm{C}_{2}-\mathrm{H}_{4} / \mathrm{A}$ & 1.08 & 1.08 & 1.08 & 1.08 \\
\hline $\mathbf{C}_{2}{ }^{\prime}-\mathbf{H}_{4}^{\prime} / \AA$ & 1.08 & 1.08 & 1.08 & 1.08 \\
\hline $\mathrm{C}_{1}-\mathrm{C}_{2}-\mathrm{Cl} /{ }^{\circ}$ & 110.5 & 110.2 & 110.2 & 110.9 \\
\hline $\mathrm{C}_{1}^{\prime}-\mathrm{C}_{2}^{\prime}-\mathrm{Cl}^{\prime} /{ }^{\circ}$ & 110.5 & 110.6 & 110.6 & 109.7 \\
\hline $\mathbf{S}-\mathbf{C}_{1}-\mathbf{C}_{2} /^{\circ}$ & 111.5 & 111.2 & 111.3 & 110.8 \\
\hline $\mathbf{S}-\mathbf{C}_{1}{ }^{\prime}-\mathbf{C}_{2}{ }^{\prime} /{ }^{\circ}$ & 111.5 & 110.7 & 110.7 & 111.6 \\
\hline $\mathrm{C}_{1}{ }^{\prime}-\mathrm{S}-\mathrm{C}_{1} /^{\circ}$ & 101.9 & 102.6 & 102.6 & 102.1 \\
\hline Cl- $C_{2}-C_{1}-\mathbf{S}\left(\phi_{1}\right) /^{\circ}$ & 180.0 & 179.3 & -179.3 & -179.3 \\
\hline $\mathrm{C}_{2}-\mathrm{C}_{1}-\mathrm{S}-\mathrm{C}_{1}{ }^{\prime}\left(\phi_{2}\right) /^{\circ}$ & 82.5 & 82.6 & 82.6 & 76.2 \\
\hline$C_{2}{ }^{\prime}-C_{1}{ }^{\prime}-S-C_{1}\left(\phi_{3}\right) /^{\circ}$ & 82.5 & 82.20 & 82.2 & 80.9 \\
\hline $\mathrm{Cl}^{\prime}-\mathrm{C}_{2}{ }^{\prime}-\mathrm{C}_{1}{ }^{\prime}-\mathrm{S}\left(\phi_{4}\right) /^{\circ}$ & 180.0 & 173.5 & 173.5 & 178.9 \\
\hline
\end{tabular}


Table S6: Structural parameters of the most stable SM. $n \mathrm{H}_{2} \mathrm{O}$ clusters optimised using B3LYPD3/6-311++G(2d,2p).

\begin{tabular}{|c|c|c|c|c|}
\hline Bonds & Isolated A & A1-1 & A2-1 & A3-1 \\
\hline $\mathrm{C}_{2}-\mathrm{Cl} / \AA$ & 1.83 & 1.84 & 1.85 & 1.84 \\
\hline $\mathbf{C}_{2}^{\prime}-\mathbf{C l}^{\prime} / \AA ̊ ̊$ & 1.83 & 1.84 & 1.84 & 1.84 \\
\hline $\mathbf{C}_{1}-\mathbf{C}_{2} / \AA$ & 1.52 & 1.51 & 1.51 & 1.51 \\
\hline $\mathbf{C}_{1}^{\prime}-\mathbf{C}_{2}^{\prime} / \AA$ & 1.52 & 1.51 & 1.51 & 1.51 \\
\hline $\mathbf{S}-\mathbf{C}_{1} / \AA$ & 1.84 & 1.84 & 1.84 & 1.83 \\
\hline $\mathbf{S}-\mathbf{C}_{1}^{\prime} / \mathbf{A}$ & 1.84 & 1.84 & 1.84 & 1.84 \\
\hline $\mathbf{C}_{1}-\mathbf{H}_{1} / \AA$ & 1.09 & 1.09 & 1.09 & 1.09 \\
\hline $\mathbf{C}_{1}{ }^{\prime}-\mathbf{H}_{1}{ }^{\prime} / \mathbf{A}$ & 1.09 & 1.09 & 1.09 & 1.09 \\
\hline $\mathbf{C}_{1}-\mathbf{H}_{2} / \AA$ & 1.09 & 1.09 & 1.09 & 1.09 \\
\hline $\mathbf{C}_{1}{ }^{\prime}-\mathbf{H}_{2}{ }^{\prime} / \mathbf{A}$ & 1.09 & 1.09 & 1.09 & 1.09 \\
\hline $\mathrm{C}_{2}-\mathrm{H}_{3} / \AA$ & 1.09 & 1.08 & 1.08 & 1.08 \\
\hline $\mathbf{C}_{2}^{\prime}-\mathbf{H}_{3}^{\prime} / \AA$ & 1.09 & 1.08 & 1.08 & 1.08 \\
\hline $\mathrm{C}_{2}-\mathrm{H}_{4} / \AA$ & 1.08 & 1.08 & 1.08 & 1.08 \\
\hline $\mathrm{C}_{2}^{\prime}-\mathbf{H}_{4}^{\prime} / \mathrm{A}$ & 1.08 & 1.08 & 1.08 & 1.08 \\
\hline $\mathrm{C}_{1}-\mathrm{C}_{2}-\mathrm{Cl} /{ }^{\circ}$ & 110.2 & 110.1 & 110.0 & 110.2 \\
\hline $\mathrm{C}_{1}^{\prime}-\mathrm{C}_{2}^{\prime}-\mathrm{Cl}^{\prime} /{ }^{\circ}$ & 110.2 & 110.1 & 110.1 & 110.1 \\
\hline $\mathbf{S}-\mathbf{C}_{1}-\mathbf{C}_{2} /^{\circ}$ & 111.5 & 111.4 & 111.1 & 111.3 \\
\hline $\mathbf{S}-\mathrm{C}_{1}{ }^{\prime}-\mathrm{C}_{2}, / O$ & 111.5 & 111.4 & 111.0 & 111.1 \\
\hline $\mathrm{C}_{1}^{\prime}-\mathrm{S}-\mathrm{C}_{1} /^{\circ}$ & 101.0 & 101.0 & 102.0 & 102.2 \\
\hline $\mathrm{Cl}-\mathrm{C}_{2}-\mathrm{C}_{1}-\mathrm{S}\left(\phi_{1}\right) /^{\circ}$ & -179.4 & -175.4 & -177.8 & -178.0 \\
\hline $\mathrm{C}_{2}-\mathrm{C}_{1}-\mathrm{S}-\mathrm{C}_{1}^{\prime}\left(\phi_{2}\right) /^{\circ}$ & 80.4 & 76.3 & 78.8 & 74.7 \\
\hline $\mathrm{C}_{2}{ }^{\prime}-\mathrm{C}_{1}{ }^{\prime}-\mathrm{S}-\mathrm{C}_{1}\left(\phi_{3}\right) /^{\circ}$ & 80.4 & 76.3 & 78.0 & 78.1 \\
\hline $\mathrm{Cl}^{\prime}-\mathrm{C}_{2}{ }^{\prime}-\mathrm{C}_{1}{ }^{\prime}-\mathrm{S}\left(\phi_{4}\right) /^{\circ}$ & -179.4 & -175.4 & 172.7 & 170.3 \\
\hline
\end{tabular}


Table S7: Structural parameters of the most stable SM. $n \mathrm{H}_{2} \mathrm{O}$ clusters optimised using MP2/6$311++\mathrm{G}(2 \mathrm{~d}, 2 \mathrm{p})$

\begin{tabular}{|c|c|c|c|c|}
\hline Bonds & Isolated A & A1-1 & A2-1 & A3-1 \\
\hline $\mathrm{C}_{2}-\mathrm{Cl} / \AA$ & 1.80 & 1.81 & 1.82 & 1.84 \\
\hline $\mathbf{C}_{2}{ }^{\prime}-\mathbf{C l}^{\prime} / \mathbf{A}$ & 1.80 & 1.81 & 1.81 & 1.84 \\
\hline $\mathbf{C}_{1}-\mathbf{C}_{2} / \AA$ & 1.51 & 1.51 & 1.51 & 1.51 \\
\hline $\mathbf{C}_{1}^{\prime}-\mathbf{C}_{2}^{\prime} / \mathbf{A}$ & 1.51 & 1.51 & 1.51 & 1.51 \\
\hline $\mathbf{S}-\mathbf{C}_{1} / \mathbf{A}$ & 1.82 & 1.82 & 1.82 & 1.83 \\
\hline $\mathbf{S}-\mathbf{C}_{1}^{\prime} / \mathbf{A}$ & 1.82 & 1.82 & 1.82 & 1.84 \\
\hline $\mathbf{C}_{1}-\mathbf{H}_{1} / \AA$ & 1.09 & 1.09 & 1.09 & 1.09 \\
\hline $\mathbf{C}_{1}{ }^{\prime}-\mathbf{H}_{1}{ }^{\prime} / \mathbf{A}$ & 1.09 & 1.09 & 1.09 & 1.09 \\
\hline $\mathbf{C}_{1}-\mathbf{H}_{2} / \AA$ & 1.09 & 1.08 & 1.08 & 1.09 \\
\hline $\mathbf{C}^{\prime}{ }^{\prime}-\mathbf{H}_{2}^{\prime} / \mathbf{A}$ & 1.09 & 1.08 & 1.08 & 1.09 \\
\hline $\mathrm{C}_{2}-\mathrm{H}_{3} / \AA$ & 1.08 & 1.08 & 1.08 & 1.08 \\
\hline $\mathbf{C}_{2}^{\prime}-\mathbf{H}_{3^{\prime}} / \mathbf{A}$ & 1.08 & 1.08 & 1.08 & 1.08 \\
\hline $\mathrm{C}_{2}-\mathrm{H}_{4} / \mathrm{A}$ & 1.08 & 1.08 & 1.08 & 1.08 \\
\hline $\mathbf{C}_{2}^{\prime}-\mathbf{H}_{4}^{\prime} / \mathbf{A}$ & 1.08 & 1.08 & 1.08 & 1.08 \\
\hline $\mathrm{C}_{1}-\mathrm{C}_{2}-\mathrm{Cl} /{ }^{\circ}$ & 110.2 & 110.1 & 110.1 & 110.1 \\
\hline $\mathrm{C}_{1}{ }^{\prime}-\mathrm{C}_{2}{ }^{\prime}-\mathrm{Cl}^{\prime} /^{\circ}$ & 110.2 & 110.1 & 109.9 & 110.2 \\
\hline $\mathbf{S}-\mathbf{C}_{1}-\mathbf{C}_{2} /^{\circ}$ & 111.0 & 110.9 & 110.7 & 111.1 \\
\hline $\mathbf{S}-\mathbf{C}_{1}{ }^{\prime}-\mathbf{C}_{2}{ }^{\prime} /^{\circ}$ & 111.0 & 110.9 & 110.8 & 111.3 \\
\hline $\mathrm{C}_{1}{ }^{\prime}-\mathrm{S}-\mathrm{C}_{1} /^{\circ}$ & 99.4 & 99.7 & 100.8 & 102.2 \\
\hline $\mathrm{Cl}-\mathrm{C}_{2}-\mathrm{C}_{1}-\mathrm{S}\left(\phi_{1}\right) /^{\circ}$ & -178.6 & -176.6 & -178.2 & -178.0 \\
\hline $\mathrm{C}_{2}-\mathrm{C}_{1}-\mathrm{S}-\mathrm{C}_{1^{\prime}}(\phi 2) /^{\circ}$ & 81.2 & 77.1 & 77.6 & 78.1 \\
\hline $\mathrm{C}_{2}^{\prime}-\mathrm{C}_{1}{ }^{\prime}-\mathrm{S}-\mathrm{C}_{1}(\boldsymbol{\phi} \mathbf{3}) /^{\circ}$ & 81.2 & 77.1 & 78.4 & 74.7 \\
\hline $\mathrm{Cl}^{\prime}-\mathrm{C}_{2}{ }^{\prime}-\mathrm{C}_{1}{ }^{\prime}-\mathrm{S}(\phi 4) /^{\circ}$ & -178.6 & -176.6 & 173.8 & 170.3 \\
\hline
\end{tabular}


Table S8: HB lengths and angles for the most stable SM. $n \mathrm{H}_{2} \mathrm{O}$ complexes in each generation using B3LYP/6-311++G(2d,2p)

\begin{tabular}{|c|c|c|}
\hline HBs & HB length & HB angle \\
\hline \multicolumn{3}{|c|}{ A1-1 } \\
\hline $\mathrm{C}_{1}-\mathrm{H}_{2} \cdots \mathrm{O}_{1}-\mathrm{H}_{5}$ & 2.60 & 123.5 \\
\hline $\mathrm{C}_{2}-\mathrm{H}_{4} \cdots \mathrm{O}_{1}-\mathrm{H}_{5}$ & 2.79 & 106.6 \\
\hline $\mathrm{C}_{1}^{\prime}-\mathrm{H}_{2}{ }^{\prime} \cdots \mathrm{O}_{1}-\mathrm{H}_{5}$ & 2.84 & 120.4 \\
\hline $\mathrm{C}_{2}{ }^{\prime}-\mathrm{H}_{4}{ }^{\prime} \cdots \mathrm{O}_{1}-\mathrm{H}_{5}$ & 2.80 & 119.2 \\
\hline $\mathrm{O}_{1}-\mathrm{H}_{6} \cdots \mathrm{Cl}$ & 2.72 & 128.0 \\
\hline \multicolumn{3}{|c|}{ A2-1 } \\
\hline $\mathbf{O}_{1}-\mathbf{H}_{6} \cdots \mathbf{O}_{2}-\mathbf{H}_{7}$ & 1.88 & 162.9 \\
\hline $\mathrm{C}_{1}-\mathrm{H}_{2} \cdots \mathrm{O}_{1}-\mathrm{H}_{5}$ & 2.62 & 128.7 \\
\hline $\mathrm{C}_{2}-\mathrm{H}_{4} \cdots \mathrm{O}_{1}-\mathrm{H}_{5}$ & 2.95 & 114.1 \\
\hline $\mathrm{C}_{1}{ }^{\prime}-\mathrm{H}_{2}{ }^{\prime} \cdots \mathrm{O}_{1}-\mathrm{H}_{5}$ & 2.60 & 121.4 \\
\hline $\mathrm{C}_{2}^{\prime}-\mathrm{H}_{4}{ }^{\prime} \cdots \mathrm{O}_{1}-\mathrm{H}_{5}$ & 2.66 & 113.1 \\
\hline $\mathrm{O}_{2}-\mathrm{H}_{7} \cdots \mathrm{Cl}^{\prime}-\mathrm{C}_{2}^{\prime}$ & 2.41 & 164.9 \\
\hline \multicolumn{3}{|c|}{ A3-1 } \\
\hline $\mathbf{O}_{1}-\mathbf{H}_{5} \cdots \mathbf{O}_{2}-\mathbf{H}_{7}$ & 1.88 & 170.4 \\
\hline $\mathrm{O}_{2}-\mathrm{H}_{7} \cdots \mathrm{O}_{3}-\mathrm{H}_{10}$ & 1.81 & 167.1 \\
\hline $\mathrm{C}_{1}-\mathrm{H}_{2} \cdots \mathrm{O}_{3}-\mathrm{H}_{10}$ & 2.55 & 175.8 \\
\hline $\mathrm{C}_{2}-\mathrm{H}_{4} \cdots \mathrm{O}_{1}-\mathrm{H}_{5}$ & 2.25 & 171.5 \\
\hline $\mathrm{C}_{1}^{\prime}-\mathrm{H}_{2}{ }^{\prime} \cdots \mathrm{O}_{1}-\mathrm{H}_{5}$ & 2.38 & 175.5 \\
\hline $\mathrm{O}_{3}-\mathrm{H}_{9} \cdots \mathrm{Cl}-\mathrm{C}_{2}$ & 2.40 & 164.1 \\
\hline $\mathrm{O}_{2}-\mathrm{H}_{8} \cdots \mathrm{Cl}^{\prime}-\mathrm{C}_{2}{ }^{\prime}$ & 2.59 & 157.0 \\
\hline
\end{tabular}


Table S9: HB lengths and angles for the most stable SM. $n \mathrm{H}_{2} \mathrm{O}$ complexes in each generation using B3LYP-D3/6-311++G(2d,2p)

\begin{tabular}{|c|c|c|}
\hline HBs & HB length & HB angle \\
\hline \multicolumn{3}{|c|}{ A1-1 } \\
\hline $\mathrm{C}_{1}-\mathrm{H}_{2} \cdots \mathrm{O}_{1}-\mathrm{H}_{5}$ & 2.56 & 117.8 \\
\hline $\mathrm{C}_{2}-\mathrm{H}_{4} \cdots \mathrm{O}_{1}-\mathrm{H}_{5}$ & 2.56 & 110.9 \\
\hline $\mathrm{C}_{1}^{\prime}-\mathrm{H}_{2}^{\prime} \cdots \mathrm{O}_{1}-\mathrm{H}_{5}$ & 2.56 & 117.8 \\
\hline $\mathrm{C}_{2}^{\prime}-\mathrm{H}_{4}^{\prime} \cdots \mathrm{O}_{1}-\mathrm{H}_{5}$ & 2.56 & 110.9 \\
\hline $\mathrm{O}_{1}-\mathrm{H}_{5} \cdots \mathrm{Cl}$ & 2.93 & 110.4 \\
\hline $\mathrm{O}_{1}-\mathrm{H}_{6} \cdots \mathrm{Cl}^{\prime}$ & 2.93 & 110.4 \\
\hline \multicolumn{3}{|c|}{ A2-1 } \\
\hline $\mathbf{O}_{1}-\mathbf{H}_{5} \cdots \mathbf{O}_{2}-\mathbf{H}_{7}$ & 1.87 & 161.5 \\
\hline $\mathrm{C}_{1}-\mathbf{H}_{2} \cdots \mathrm{O}_{1}-\mathbf{H}_{5}$ & 2.59 & 120.4 \\
\hline $\mathrm{C}_{2}-\mathrm{H}_{4} \cdots \mathrm{O}_{1}-\mathrm{H}_{5}$ & 2.58 & 119.9 \\
\hline $\mathrm{C}_{1}^{\prime}-\mathrm{H}_{2}^{\prime} \cdots \mathrm{O}_{1}-\mathrm{H}_{5}$ & 2.49 & 119.4 \\
\hline $\mathrm{C}_{2}^{\prime}-\mathrm{H}_{4}^{\prime} \cdots \mathrm{O}_{1}-\mathrm{H}_{5}$ & 2.55 & 110.4 \\
\hline $\mathrm{O}_{1}-\mathrm{H}_{6} \cdots \mathrm{Cl}^{\prime}-\mathrm{C}_{2}{ }^{\prime}$ & 2.79 & 122.0 \\
\hline $\mathrm{O}_{2}-\mathrm{H}_{7} \cdots \mathrm{Cl}-\mathrm{C}_{2}$ & 2.38 & 162.6 \\
\hline \multicolumn{3}{|c|}{ A3-1 } \\
\hline $\mathrm{O}_{1}-\mathrm{H}_{6} \cdots \mathrm{O}_{2}-\mathrm{H}_{7}$ & 1.81 & 157.1 \\
\hline $\mathrm{O}_{2}-\mathrm{H}_{7} \cdots \mathrm{O}_{3}-\mathrm{H}_{9}$ & 2.06 & 140.0 \\
\hline $\mathrm{O}_{3}-\mathrm{H}_{9} \cdots \mathrm{O}_{1}-\mathrm{H}_{6}$ & 1.91 & 151.8 \\
\hline $\mathrm{C}_{1}-\mathrm{H}_{2} \cdots \mathrm{O}_{1}-\mathrm{H}_{5}$ & 2.53 & 155.5 \\
\hline $\mathrm{C}_{2}^{\prime}-\mathrm{H}_{4}^{\prime} \cdots \mathrm{O}_{1}-\mathrm{H}_{5}$ & 2.38 & 152.6 \\
\hline $\mathrm{C}_{1}^{\prime}-\mathrm{H}_{2}^{\prime} \cdots \mathrm{O}_{2}-\mathrm{H}_{7}$ & 2.55 & 147.7 \\
\hline $\mathrm{C}_{2}-\mathbf{H}_{4} \cdots \mathbf{O}_{2}-\mathbf{H}_{7}$ & 2.48 & 138.8 \\
\hline $\mathrm{O}_{3}-\mathrm{H}_{10} \cdots \mathrm{Cl}-\mathrm{C}_{2}$ & 2.44 & 153.4 \\
\hline
\end{tabular}


Table S10: HB lengths and angles for the most stable SM. $n \mathrm{H}_{2} \mathrm{O}$ complexes in each generation using MP2/6-311++G(2d,2p)

\begin{tabular}{|c|c|c|}
\hline HBs & HB length & HB angle \\
\hline \multicolumn{3}{|c|}{ A1-1 } \\
\hline $\mathrm{C}_{1}-\mathrm{H}_{2} \cdots \mathrm{O}_{1}-\mathrm{H}_{5}$ & 2.55 & 119.1 \\
\hline $\mathrm{C}_{2}-\mathrm{H}_{4} \cdots \mathrm{O}_{1}-\mathrm{H}_{5}$ & 2.60 & 109.8 \\
\hline $\mathrm{C}_{1}^{\prime}-\mathrm{H}_{2}^{\prime} \cdots \mathrm{O}_{1}-\mathrm{H}_{5}$ & 2.55 & 119.1 \\
\hline $\mathrm{C}_{2}^{\prime}-\mathrm{H}_{4}^{\prime} \cdots \mathrm{O}_{1}-\mathrm{H}_{5}$ & 2.60 & 109.8 \\
\hline $\mathrm{O}_{1}-\mathrm{H}_{5} \cdots \mathrm{Cl}$ & 2.96 & 108.4 \\
\hline $\mathrm{O}_{1}-\mathrm{H}_{6} \cdots \mathrm{Cl}^{\prime}$ & 2.96 & 108.4 \\
\hline \multicolumn{3}{|c|}{ A2-1 } \\
\hline $\mathbf{O}_{1}-\mathbf{H}_{5} \cdots \mathbf{O}_{2}-\mathbf{H}_{7}$ & 1.89 & 159.2 \\
\hline $\mathrm{C}_{1}-\mathrm{H}_{2} \cdots \mathrm{O}_{1}-\mathrm{H}_{5}$ & 2.66 & 117.7 \\
\hline $\mathrm{C}_{2}-\mathrm{H}_{4} \cdots \mathrm{O}_{1}-\mathrm{H}_{5}$ & 2.54 & 121.1 \\
\hline $\mathrm{C}_{1}^{\prime}-\mathrm{H}_{2}^{\prime} \cdots \mathrm{O}_{1}-\mathrm{H}_{5}$ & 2.44 & 121.6 \\
\hline $\mathrm{C}_{2}^{\prime}-\mathrm{H}_{4}^{\prime} \cdots \mathrm{O}_{1}-\mathrm{H}_{5}$ & 2.61 & 107.3 \\
\hline $\mathrm{O}_{1}-\mathrm{H}_{6} \cdots \mathrm{Cl}^{\prime}-\mathrm{C}_{2}^{\prime}$ & 2.74 & 122.8 \\
\hline $\mathrm{O}_{2}-\mathrm{H}_{7} \cdots \mathrm{Cl}-\mathrm{C}_{2}$ & 2.37 & 163.2 \\
\hline \multicolumn{3}{|c|}{ A3-1 } \\
\hline $\mathrm{O}_{1}-\mathrm{H}_{5} \cdots \mathrm{O}_{2}-\mathrm{H}_{7}$ & 1.81 & 157.1 \\
\hline $\mathrm{O}_{2}-\mathrm{H}_{7} \cdots \mathrm{O}_{3}-\mathrm{H}_{9}$ & 2.06 & 140.0 \\
\hline $\mathrm{O}_{3}-\mathrm{H}_{9} \cdots \mathrm{O}_{1}-\mathrm{H}_{5}$ & 1.91 & 151.8 \\
\hline $\mathrm{C}_{1}-\mathbf{H}_{2} \cdots \mathrm{O}_{1}-\mathrm{H}_{5}$ & 2.53 & 155.5 \\
\hline $\mathrm{C}_{2}^{\prime}-\mathrm{H}_{4}^{\prime} \cdots \mathrm{O}_{1}-\mathrm{H}_{5}$ & 2.38 & 152.6 \\
\hline $\mathrm{C}_{1}-\mathbf{H}_{2} \cdots \mathbf{O}_{2}-\mathbf{H}_{7}$ & 2.48 & 138.8 \\
\hline $\mathrm{C}_{2}-\mathrm{H}_{4} \cdots \mathrm{O}_{2}-\mathrm{H}_{7}$ & 2.55 & 147.7 \\
\hline $\mathrm{O}_{3}-\mathrm{H}_{10} \cdots \mathrm{Cl}-\mathrm{C}_{2}$ & 2.44 & 153.4 \\
\hline
\end{tabular}




\section{Effect of microhydration on the infrared wavenumbers of SM and water}

The infrared (IR) wavenumbers of the most stable complexes of each generation, A1-1, A2-1 and A3-1 using each of the three different methods are shown in Tables S11 to S13. Scaling of the calculated values have been performed for the results obtained using B3LYP/6-311++G(2d,2p) only, as the scaling factors for B3LYP-D3 and MP2 were not available.

Table S11: Selected vibrational wavenumbers of the most stable complexes obtained using B3LYP /6-311++G(2d,2p) and scaled by $0.963^{[66]}$. Values in parentheses are the changes in vibrational wavenumbers relative to the isolated free $\mathbf{A}$ and water cluster molecules

\begin{tabular}{|c|c|c|c|c|c|}
\hline \multirow{2}{*}{$\begin{array}{l}\text { Bonds and modes } \\
\text { Water }\end{array}$} & \multicolumn{5}{|c|}{ IR wavenumber $/ \mathrm{cm}^{-1}$} \\
\hline & $\mathbf{A}$ & $\left(\mathbf{H}_{2} \mathbf{O}\right)_{n}$ & A1-1 & A2-1 & A3-1 \\
\hline$v($ as $) \mathrm{O}-\mathrm{H}$ & - & 3779 & $3759(-20)$ & - & - \\
\hline$v(\mathrm{~s}) \mathrm{O}-\mathrm{H}$ & - & 3681 & $3650(-31)$ & - & - \\
\hline$\delta(\mathrm{s}) \mathrm{H}-\mathrm{O}-\mathrm{H}$ & - & 1578 & $1572(-7)$ & - & - \\
\hline Water dimer & - & - & & & \\
\hline$v($ as $) \mathrm{O}-\mathrm{H}$ & - & 3767 & - & $3739(-28)$ & - \\
\hline$v($ as $) \mathrm{O}^{\prime}-\mathrm{H}^{\prime}$ & - & 3751 & - & $3731(-20)$ & - \\
\hline$v(\mathrm{~s}) \mathrm{O}-\mathrm{H}$ & - & 3673 & - & $3583(-90)$ & - \\
\hline$v(\mathrm{~s}) \mathrm{O}^{\prime}-\mathrm{H}^{\prime}$ & - & 3568 & - & $3489(-79)$ & - \\
\hline$\delta(\mathrm{s}) \mathrm{H}-\mathrm{O}-\mathrm{H}$ & - & 1599 & - & $1604(6)$ & - \\
\hline$\delta(\mathrm{s}) \mathrm{H}^{\prime}-\mathrm{O}^{\prime}-\mathrm{H}^{\prime}$ & - & 1580 & - & $1587(7)$ & - \\
\hline Water trimer & & & & - & \\
\hline$v($ as $) \mathrm{O}-\mathrm{H}$ & - & 3745 & - & - & $3745(0)$ \\
\hline$v($ as $) \mathrm{O}^{\prime}-\mathrm{H}^{\prime}$ & - & 3744 & - & - & $3735(-10)$ \\
\hline$v($ as $) \mathrm{O}^{\prime \prime}-\mathrm{H}^{\prime \prime}$ & - & 3740 & - & - & $3664(-76)$ \\
\hline$v(\mathrm{~s}) \mathrm{O}-\mathrm{H}$ & - & 3496 & - & - & $3560(65)$ \\
\hline$v(\mathrm{~s}) \mathrm{O}^{\prime}-\mathrm{H}^{\prime}$ & - & 3485 & - & - & $3492(7)$ \\
\hline$v(\mathrm{~s}) \mathrm{O} "-\mathrm{H}^{\prime \prime}$ & - & 3426 & - & - & $3371(-56)$ \\
\hline$\delta(\mathrm{s}) \mathrm{H}-\mathrm{O}-\mathrm{H}$ & - & 1613 & - & - & $1617(4)$ \\
\hline$\delta(\mathrm{s}) \mathrm{H}^{\prime}-\mathrm{O}^{\prime}-\mathrm{H}^{\prime}$ & - & 1594 & - & - & $1603(10)$ \\
\hline$\delta(\mathrm{s}) \mathrm{H} "{ }^{\prime \prime}-\mathrm{O} "-\mathrm{H}^{\prime \prime}$ & - & 1591 & - & - & $1590(-1)$ \\
\hline \multicolumn{6}{|l|}{$\mathrm{SM} . n \mathrm{H}_{2} \mathrm{O}$} \\
\hline$v(\mathrm{~s}) \mathrm{Cl}-\mathrm{C}$ & 647 & - & - & $625(-22)$ & $624(-23)$ \\
\hline$v($ as $) \mathrm{Cl}-\mathrm{C}$ & 662 & & $\begin{array}{l}638(-24) \\
650(-12)\end{array}$ & $646(-15)$ & $639(-22)$ \\
\hline$v(\mathrm{~s}) \mathrm{C}-\mathrm{H}($ external $\mathrm{C})$ & 2993 & - & $\begin{array}{l}2999(6) \\
3002(9)\end{array}$ & $\begin{array}{l}2999(6) \\
3002(9)\end{array}$ & $\begin{array}{l}2994(1), \\
2995(2)\end{array}$ \\
\hline$v(\mathrm{~s}) \mathrm{C}-\mathrm{H}$ (internal C) & 2965 & - & $\begin{array}{l}2970(5) \\
2073(8)\end{array}$ & $\begin{array}{l}2970(5) \\
2072(7)\end{array}$ & $\begin{array}{r}2964(-1) \\
2966(1)\end{array}$ \\
\hline
\end{tabular}


Table S12: Selected and unscaled vibrational wavenumbers of the most stable complexes obtained using B3LYP-D3/6-311++G(2d,2p). Values in parentheses are the changes in vibrational wavenumbers relative to the isolated free $\mathbf{A}$ and water cluster molecules

\begin{tabular}{|c|c|c|c|c|c|}
\hline \multirow{2}{*}{$\begin{array}{l}\text { Bonds and modes } \\
\text { Water }\end{array}$} & \multicolumn{5}{|c|}{ IR wavenumber $/ \mathrm{cm}^{-1}$} \\
\hline & $\mathbf{A}$ & $\left(\mathbf{H}_{2} \mathbf{O}\right)_{n}$ & A1-1 & A2-1 & A3-1 \\
\hline$v($ as $) \mathrm{O}-\mathrm{H}$ & - & 3924 & $3907(-17)$ & - & - \\
\hline$v(s) \mathrm{O}-\mathrm{H}$ & - & 3821 & $3800(-21)$ & - & - \\
\hline$\delta(\mathrm{s}) \mathrm{H}-\mathrm{O}-\mathrm{H}$ & - & 1639 & $1618(-21)$ & - & - \\
\hline Water dimer & - & & & & \\
\hline$v($ as $) \mathrm{O}-\mathrm{H}$ & - & 3913 & - & $3883(-30)$ & - \\
\hline$v($ as $) \mathrm{O}^{\prime}-\mathrm{H}^{\prime}$ & - & 3895 & - & $3861(-34)$ & - \\
\hline$v(\mathrm{~s}) \mathrm{O}-\mathrm{H}$ & - & 3815 & - & $3719(-96)$ & - \\
\hline$v(\mathrm{~s}) \mathrm{O}^{\prime}-\mathrm{H}^{\prime}$ & - & 3707 & - & $3622(-85)$ & - \\
\hline$\delta(\mathrm{s}) \mathrm{H}-\mathrm{O}-\mathrm{H}$ & - & 1660 & - & $1667(7)$ & - \\
\hline$\delta(\mathrm{s}) \mathrm{H}^{\prime}-\mathrm{O}^{\prime}-\mathrm{H}^{\prime}$ & - & 1641 & - & $1648(7)$ & - \\
\hline \multicolumn{6}{|l|}{ Water trimer } \\
\hline$v($ as $) \mathrm{O}-\mathrm{H}$ & - & 3891 & - & - & $3879(-12)$ \\
\hline$v($ as $) \mathrm{O}^{\prime}-\mathrm{H}^{\prime}$ & - & 3889 & - & - & $3871(-18)$ \\
\hline$v($ as)O"'-H" & - & 3885 & - & - & $3792(-93)$ \\
\hline$v(\mathrm{~s}) \mathrm{O}-\mathrm{H}$ & - & 3627 & - & - & $3707(80)$ \\
\hline$v(s) \mathrm{O}^{\prime}-\mathrm{H}^{\prime}$ & - & 3617 & - & - & $3610(-7)$ \\
\hline$v(\mathrm{~s}) \mathrm{O}^{\prime \prime}-\mathrm{H}^{\prime \prime}$ & - & 3553 & - & - & $3448(-105)$ \\
\hline$\delta(\mathrm{s}) \mathrm{H}-\mathrm{O}-\mathrm{H}$ & - & 1674 & - & - & $1682(8)$ \\
\hline$\delta(\mathrm{s}) \mathrm{H}^{\prime}-\mathrm{O}^{\prime}-\mathrm{H}^{\prime}$ & - & 1655 & - & - & 1669 (14) \\
\hline$\delta(\mathrm{s}) \mathrm{H}^{\prime \prime}-\mathrm{O} "-\mathrm{H}^{\prime \prime}$ & - & 1652 & - & - & $1653(1)$ \\
\hline \multicolumn{6}{|l|}{ SM.nH2O } \\
\hline$v(\mathrm{~s}) \mathrm{Cl}-\mathrm{C}$ & 673 & - & $664(-27)$ & $648(-25)$ & $654(-19)$ \\
\hline$v($ as $) \mathrm{Cl}-\mathrm{C}$ & 685 & - & $694(-27)$ & $666(-19)$ & $\begin{array}{l}659(-19) \\
666(-19)\end{array}$ \\
\hline$v(s) C-H($ external C) & $\begin{array}{l}3102 \\
3103\end{array}$ & - & $3045(16)$ & $\begin{array}{l}3112(10), \\
3114(11)\end{array}$ & $\begin{array}{l}3107(5), \\
3112(9)\end{array}$ \\
\hline$v(\mathrm{~s}) \mathrm{C}-\mathrm{H}$ (internal C) & $\begin{array}{l}3076 \\
3078\end{array}$ & - & $3021(18)$ & $\begin{array}{l}3082(6) \\
3086(8)\end{array}$ & $\begin{array}{l}3078(2) \\
3084(6)\end{array}$ \\
\hline
\end{tabular}


Table S13: Unscaled selected vibrational wavenumbers of the most stable complexes obtained using MP2/6-311++G(2d,2p). Values in parentheses are the changes in vibrational wavenumbers relative to the isolated free $\mathbf{A}$ and water cluster molecules.

\begin{tabular}{|c|c|c|c|c|c|}
\hline \multirow{2}{*}{$\begin{array}{l}\text { Bonds and modes } \\
\text { Water }\end{array}$} & \multicolumn{5}{|c|}{ IR wavenumber $/ \mathrm{cm}^{-1}$} \\
\hline & $\mathbf{A}$ & $\left(\mathrm{H}_{2} \mathrm{O}\right)_{n}$ & A1-1 & A2-1 & A3-1 \\
\hline$v($ as $) \mathrm{O}-\mathrm{H}$ & - & 3982 & $3848(-28)$ & - & - \\
\hline$v(\mathrm{~s}) \mathrm{O}-\mathrm{H}$ & - & 3862 & $3743(-33)$ & - & - \\
\hline$\delta(\mathrm{s}) \mathrm{H}-\mathrm{O}-\mathrm{H}$ & - & 1661 & $1580(-9)$ & - & - \\
\hline Water dimer & - & & & & \\
\hline$v($ as $) \mathrm{O}-\mathrm{H}$ & - & 3968 & - & $3842(-22)$ & - \\
\hline$v($ as $) \mathrm{O}^{\prime}-\mathrm{H}^{\prime}$ & - & 3952 & - & $3790(-68)$ & - \\
\hline$v(\mathrm{~s}) \mathrm{O}-\mathrm{H}$ & - & 3852 & - & $3700(-68)$ & - \\
\hline$v(s) \mathrm{O}^{\prime}-\mathrm{H}^{\prime}$ & - & 3769 & - & $3607(-86)$ & - \\
\hline$\delta(\mathrm{s}) \mathrm{H}-\mathrm{O}-\mathrm{H}$ & - & 1685 & - & $1607(-2)$ & - \\
\hline$\delta(\mathrm{s}) \mathrm{H}^{\prime}-\mathrm{O}^{\prime}-\mathrm{H}^{\prime}$ & - & 1660 & - & $1588(-4)$ & - \\
\hline \multicolumn{6}{|l|}{ Water trimer } \\
\hline$v($ as $) \mathrm{O}-\mathrm{H}$ & - & 3941 & - & - & $3827(-21)$ \\
\hline$v($ as $) \mathrm{O}^{\prime}-\mathrm{H}^{\prime}$ & - & 3939 & - & - & $3815(-30)$ \\
\hline$v($ as $) \mathrm{O}^{\prime \prime}-\mathrm{H}^{\prime \prime}$ & - & 3936 & - & - & $3775(-68)$ \\
\hline$v(\mathrm{~s}) \mathrm{O}-\mathrm{H}$ & - & 3705 & - & - & $3442(-170)$ \\
\hline$v(s) \mathrm{O}^{\prime}-\mathrm{H}^{\prime}$ & - & 3697 & - & - & $3573(-33)$ \\
\hline$v(\mathrm{~s}) \mathrm{O}^{\prime \prime}-\mathrm{H}^{\prime \prime}$ & - & 3638 & - & - & $3673(126)$ \\
\hline$\delta(\mathrm{s}) \mathrm{H}-\mathrm{O}-\mathrm{H}$ & - & 1700 & - & - & $1633(7)$ \\
\hline$\delta(\mathrm{s}) \mathrm{H}^{\prime}-\mathrm{O}^{\prime}-\mathrm{H}^{\prime}$ & - & 1675 & - & - & $1613(9)$ \\
\hline$\delta(\mathrm{s}) \mathrm{H}^{\prime \prime}-\mathrm{O} "-\mathrm{H}^{\prime \prime}$ & - & 1672 & - & - & $1596(-5)$ \\
\hline \multicolumn{6}{|l|}{ SM.nH2O } \\
\hline$v(\mathrm{~s}) \mathrm{Cl}-\mathrm{C}$ & 726 & - & $729(-13)$ & $754(-7)$ & $760(34)$ \\
\hline & & & & $726(-16)$ & $727(-15)$ \\
\hline$v(\mathrm{~s}) \mathrm{C}-\mathrm{H}($ external $\mathrm{C})$ & $\begin{array}{l}3142 \\
3143\end{array}$ & - & $\begin{array}{l}3150(7) \\
3150(8)\end{array}$ & $\begin{array}{l}3148(6) \\
3150(8)\end{array}$ & $3143(1), 3148(5)$ \\
\hline$v(\mathrm{~s}) \mathrm{C}-\mathrm{H}$ (internal C) & $\begin{array}{l}3113 \\
3114\end{array}$ & - & $\begin{array}{l}3117(4), \\
3121(7)\end{array}$ & $\begin{array}{l}3116(3) \\
3121(7)\end{array}$ & $\begin{array}{c}3110(-3), 3118 \\
(4)\end{array}$ \\
\hline
\end{tabular}




\section{Solvation of SM using different methods}

The solvation energy, $\Delta \mathrm{E}_{\text {Solv }}$ and free solvation energy, $\Delta \mathrm{G}_{\text {Solv }}$ of conformer $\mathbf{A}$ in PCM using M06-2X, B3LYP, B3LYP-D3 and MP2 with the 6-311++G(2d,2p) basis set has been listed in Table S14.

Table S14: The $\Delta \mathrm{E}_{\text {Solv }}$ and $\Delta \mathrm{G}_{\text {Solv }}$ of the conformer $\mathbf{A}$

\begin{tabular}{cccc}
\hline Methods & $\boldsymbol{\Delta} \mathbf{E}_{\text {Solv }} /(\mathbf{k c a l} / \mathbf{m o l})$ & $\Delta \mathbf{G}_{\text {Solv }} /(\mathbf{k c a l} / \mathbf{m o l})$ & $\begin{array}{c}\text { Corrected } \Delta \mathbf{G}_{\text {Solv }} \\
/_{(\mathbf{k c a l} / \mathbf{m o l})^{[\mathrm{aa}}}\end{array}$ \\
\hline M06-2X & -4.66 & -4.66 & -8.44 \\
B3LYP & -4.80 & -4.23 & -8.01 \\
B3LYP-D3 & -4.89 & -5.00 & -8.78 \\
MP2 & -4.41 & -4.49 & -8.27
\end{tabular}

${ }^{[a]}$ Kelly, C. P.; Cramer, C. J.; Truhlar, D. G. Aqueous Solvation Free Energies of Ions and IonWater Clusters Based on an Accurate Value for the Absolute Aqueous Solvation Free Energy of the Proton. J. Phys. Chem. B 2006, 110, 16066-16081. 